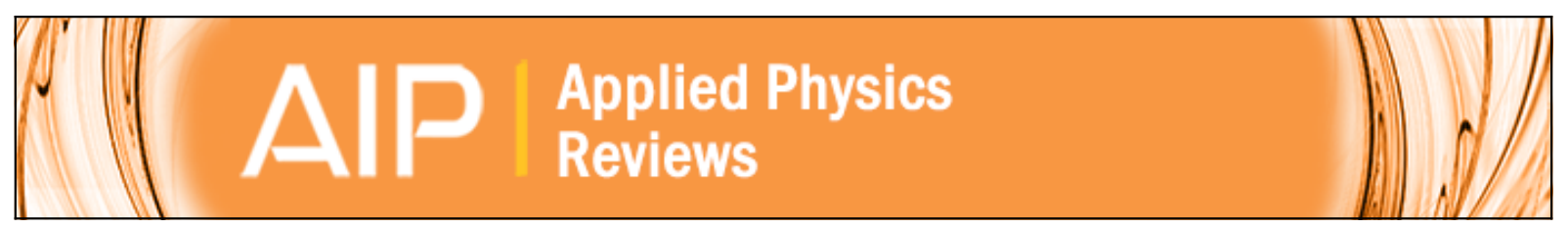

\title{
Texture in thin film silicides and germanides: A review
}

B. De Schutter, K. De Keyser, C. Lavoie, and C. Detavernier

Citation: Applied Physics Reviews 3, 031302 (2016); doi: 10.1063/1.4960122

View online: http://dx.doi.org/10.1063/1.4960122

View Table of Contents: http://scitation.aip.org/content/aip/journal/apr2/3/3?ver=pdfcov

Published by the AIP Publishing

\section{Articles you may be interested in}

Nucleation and diffusion during growth of ternary Co $1-x$ Ni $x$ Si 2 thin films studied by complementary techniques in real time

J. Appl. Phys. 104, 093533 (2008); 10.1063/1.3013449

Growth of Ti $3 \mathrm{SiC} 2$ thin films by elemental target magnetron sputtering

J. Appl. Phys. 96, 4817 (2004); 10.1063/1.1790571

Thin biaxially textured TiN films on amorphous substrates prepared by ion-beam assisted pulsed laser deposition Appl. Phys. Lett. 85, 2744 (2004); 10.1063/1.1802385

Direct Co Si 2 thin-film formation with homogeneous nanograin-size distribution by oxide-mediated silicidation J. Vac. Sci. Technol. B 22, 2299 (2004); 10.1116/1.1781660

Ti and Co silicide phase formation and transformations on deep-sub-micron gates for ULSI applications AIP Conf. Proc. 418, 439 (1998); 10.1063/1.54665

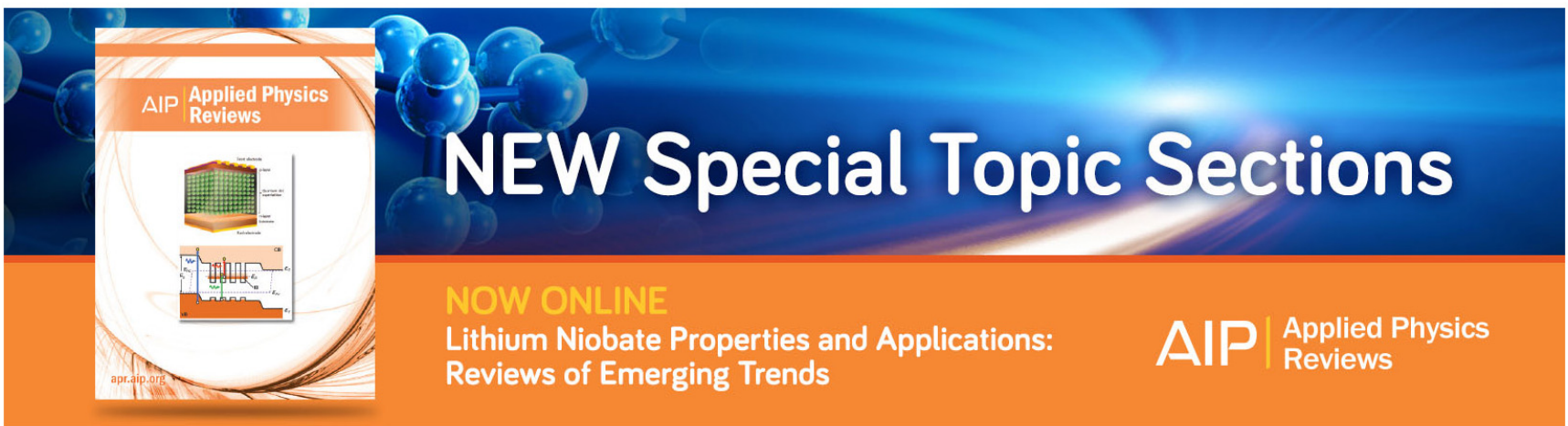




\title{
Texture in thin film silicides and germanides: A review
}

\author{
B. De Schutter, ${ }^{1, a)}$ K. De Keyser, ${ }^{1}$ C. Lavoie, ${ }^{2}$ and C. Detavernier ${ }^{1}$ \\ ${ }^{1}$ Department of Solid State Sciences, Ghent University, Ghent, Belgium \\ ${ }^{2}$ IBM Research Division, T.J. Watson Research Center, P.O. Box 218, Yorktown Heights, New York 10598, \\ USA
}

(Received 18 November 2015; accepted 14 July 2016; published online 29 August 2016)

\begin{abstract}
Silicides and germanides are compounds consisting of a metal and silicon or germanium. In the microelectronics industry, silicides are the material of choice for contacting silicon based devices (over the years, $\mathrm{CoSi}_{2}, \mathrm{C} 54-\mathrm{TiSi}_{2}$, and $\mathrm{NiSi}$ have been adopted), while germanides are considered as a top candidate for contacting future germanium based electronics. Since also strain engineering through the use of $\mathrm{Si}_{1-x} \mathrm{Ge}_{x}$ in the source/drain/gate regions of MOSFET devices is an important technique for improving device characteristics in modern Si-based microelectronics industry, a profound understanding of the formation of silicide/germanide contacts to silicon and germanium is of utmost importance. The crystallographic texture of these films, which is defined as the statistical distribution of the orientation of the grains in the film, has been the subject of scientific studies since the 1970s. Different types of texture like epitaxy, axiotaxy, fiber, or combinations thereof have been observed in such films. In recent years, it has become increasingly clear that film texture can have a profound influence on the formation and stability of silicide/germanide contacts, as it controls the type and orientation of grain boundaries (affecting diffusion and agglomeration) and the interface energy (affecting nucleation during the solid-state reaction). Furthermore, the texture also has an impact on the electrical characteristics of the contact, as the orientation and size of individual grains influences functional properties such as contact resistance and sheet resistance and will induce local variations in strain and Schottky barrier height. This review aims to give a comprehensive overview of the scientific work that has been published in the field of texture studies on thin film silicide/germanide contacts. Published by AIP Publishing.
\end{abstract}

[http://dx.doi.org/10.1063/1.4960122]

\section{TABLE OF CONTENTS}

I. INTRODUCTION

II. MEASURING TEXTURE IN THIN SILICIDE/ GERMANIDE FILMS ................. 3

A. X-ray diffraction-Pole figures ......... 3

B. Electron backscattered diffraction (EBSD) . .

III. OVERVIEW OF TEXTURE IN THIN

SILICIDE/GERMANIDE FILMS.

A. History of texture studies in thin silicide/

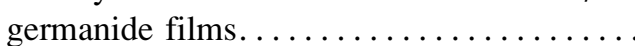

B. Pole figure based texture studies $\ldots \ldots \ldots \ldots$

C. Driving forces for texture selection ....... 1. Importance of periodicity . . . . . . .

2. Texture selection during solid state

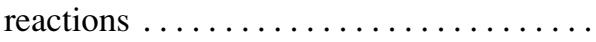

D. Texture of $\mathrm{TiSi}_{2}, \mathrm{CoSi}_{2}$, and $\mathrm{NiSi} \ldots \ldots \ldots$

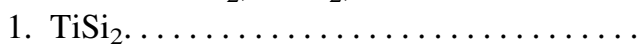

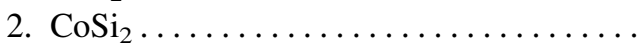

3. $\mathrm{NiSi} \ldots \ldots \ldots \ldots \ldots \ldots \ldots \ldots$

a)bob.deschutter@ugent.be
IV. INFLUENCE OF TEXTURE ON SILICIDE/ GERMANIDE FORMATION AND

PROPERTIES ................... 15

A. Influence on phase formation ......... 16

B. Influence on phase stability ........... 18

C. Influence on morphological stability ...... 19

D. Influence on electrical properties ........ 21

V. FACTORS AFFECTING TEXTURE DURING SOLID STATE REACTIONS ............ 22

A. Dopants.............................. 22

B. Lattice spacing of the substrate........ 23

C. Silicide/germanide alloying with soluble elements ....................... 24

D. Altered kinetics by adding insoluble elements: Alloys and interlayers......... 28

E. Texture inheritance from a precursor phase . 29

VI. SUMMARY AND CONCLUSIONS ......... 30

\section{INTRODUCTION}

Silicides and germanides are compounds consisting of a metal and the semiconductor silicon or germanium, respectively. Since a large fraction of the known metals react with silicon or germanium to form one or more silicides or 
germanides, a huge collection of these materials is known to exist and their properties have been the subject of many scientific studies over the past decades. As a result, a sound collection of books, book chapters, and review articles covering different aspects of silicide/germanide properties and applications are available to the reader. ${ }^{1-14}$

A very well-known use of silicides is as a contacting material in micro-electronic devices. Ever since the development of the Metal-Oxide-Semiconductor Field Effect Transistor (MOSFET) and the advent of the Si based CMOS technology, downscaling of these silicon based microelectronic devices resulted in a massive increase of their speed and complexity. The purpose of a contacting material in such a device is to ensure a good electrical connection (low contact resistance, good adhesion to $\mathrm{Si}$, high thermal stability, etc.) between the source, drain, or gate of a transistor and the interconnect lines that link it to the billions of other transistors present in a typical processor. In this context, silicides were introduced as a contacting material in the 1980s as a replacement for pure metals like Al which started to show severe issues as feature sizes decreased. ${ }^{15,16}$ Initially, a wide variety of silicide materials such as PtSi, $\mathrm{Pd}_{2} \mathrm{Si}, \mathrm{MoSi}_{2}, \mathrm{WSi}_{2}$, or $\mathrm{TaSi}_{2}$ were considered. Eventually, the industry settled on the use of $\mathrm{TiSi}_{2}$ in the early $1990 \mathrm{~s}$, but issues with the nucleation of the low-resistive C54-TiSi within the initially formed high-resistive $\mathrm{C} 49-\mathrm{TiSi}_{2}$ phase in lines narrower than $250 \mathrm{~nm}$ forced a change to $\mathrm{CoSi}_{2}{ }^{17}$ When feature sizes eventually reached sub $50 \mathrm{~nm}$ dimensions, $\mathrm{CoSi}_{2}$ started showing severe voiding issues in these narrow lines which led to the introduction of NiSi. ${ }^{18}$ In modern planar CMOS technology, NiSi modified by the addition of a small amount of $\mathrm{Pt}$ still is the contacting material of choice. ${ }^{19}$ For new device architectures such as finFETs, which allow for a further size-reduction, the criteria for contacting materials have changed drastically. These new criteria together with the availability of fast anneals have allowed the contacts to evolve back towards silicides that can withstand higher thermal budgets. ${ }^{20,21}$ For a more comprehensive historical overview on the use of silicides in microelectronics, the reader is referred to Refs. 5-7.

Silicide contacts are typically formed through a solid state reaction between a thin film of the metal of choice and the single-crystal silicon substrate by heating the metal/ silicon stack to an elevated temperature. In CMOS fabrication, this is achieved through the so-called self-aligned silicide (SALICIDE) process, where the metal is deposited over the whole planar device structure but will only react in the regions where it is exposed to the silicon during a typical formation anneal, resulting in contact formation only on the desired locations. After this formation anneal, the unreacted metal which is positioned over the dielectric regions can be selectively etched away. (For the interested reader, a detailed explanation of the SALICIDE process is provided in Ref. 7.) A schematic cross-section of a traditional CMOS device structure is provided in Fig. 1, showing the resulting silicide contacts on the source and drain regions.

In modern devices, the limitations of classical dimensional downscaling force scientists and engineers to come up with innovative techniques in order to maintain the continuous improvement of device performance. This has resulted in many material innovations such as the use of strained silicon in the channel region of the MOSFET (achieved through replacing $\mathrm{Si}$ by $\mathrm{Si}_{1-x} \mathrm{Ge}_{x}$ in the source and drain regions or through the use of nitride stressors) and the replacement of the $\mathrm{SiO}_{2} /$ polycrystalline silicon gate by a high-k/metal gate, restricting the use of silicides as contacting material to the source and drain regions. More recently, alternative channel materials like $\mathrm{Si}_{1-x} \mathrm{Ge}_{x}$ are being adopted in industry, and research is now focussing on increasing the Ge content in these channels or even adopting pure Ge as a channel material, as both $\mathrm{Si}_{1-x} \mathrm{Ge}_{x}$ and pure Ge have much higher carrier mobilities compared to $\mathrm{Si}^{22}$ In this context, germanides appear as a natural candidate to act as contacting material for the germanium-rich source and drain regions, since they can be formed in a similar self-aligned manner as mentioned above for silicides. $^{23}$

As is depicted in Fig. 1, the solid state reaction between the metal and the silicon or germanium substrate leads to the formation of a polycrystalline silicide/germanide film on top of a single crystalline substrate. In available CMOS technology, these films usually have a thickness of less than $30 \mathrm{~nm}$ and consist of a single layer of grains, as can be seen in the TEM micrograph of a NiSi film formed on Si (Fig. 1). If we want to understand and/or predict the properties of these poly-crystalline films, it is essential to have a good understanding of their microstructure. One important aspect of this

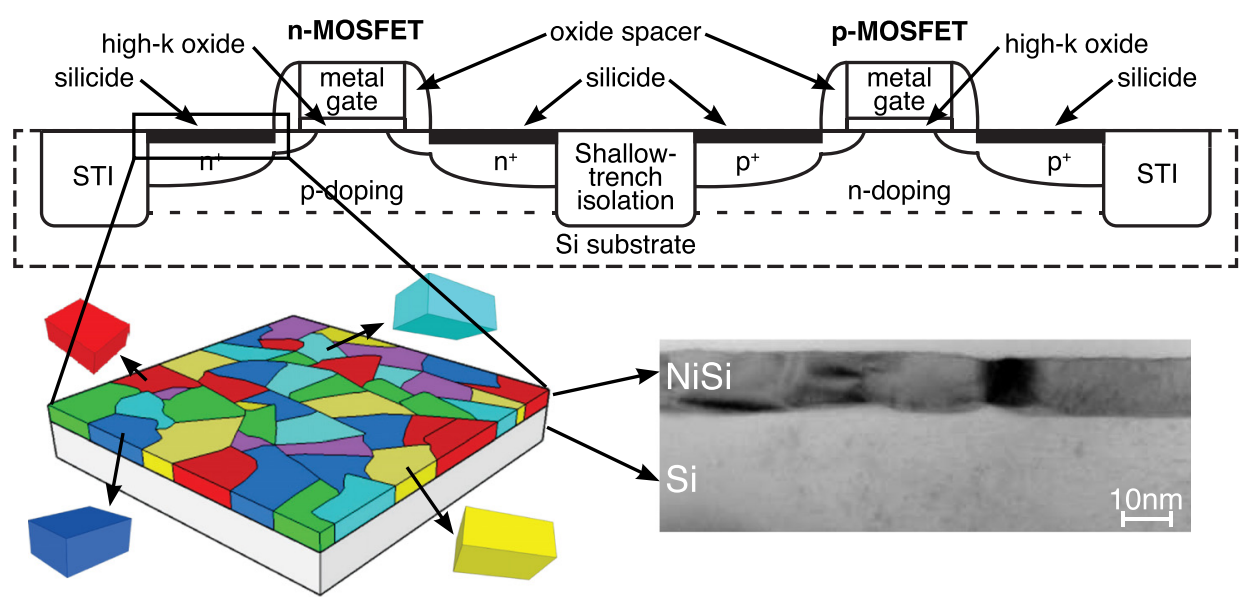

FIG. 1. Top: schematic cross-section of the typical planar CMOS n- and ptype transistors. Silicides are used to contact the source and drain regions. Pre- $45 \mathrm{~nm}$ node transistors also featured silicides to contact the gate. Bottom: Schematic detail and TEM picture for a typical polycrystalline thin silicide/germanide. Different grains in the film can exhibit different orientations with respect to the substrate. 
microstructure is the texture, which describes the orientations of the different grains and the frequency of occurrence of these different orientations within the film. This concept of texture originated from the field of bulk metallurgy, where it is known that specific preferential grain orientations can greatly influence certain properties of the materials (e.g., the strength of a rolled sheet of aluminum depends greatly on the texture of the sheet). As a detailed description of the concepts and mathematics of texture in materials research is beyond the scope of this review, the interested reader is referred to Refs. 24-26.

In this review, we will focus on the texture observed in thin silicide and germanide films formed on single crystal silicon or germanium substrates. In such films, the orientation of the grains is determined with respect to the reference frame of the single crystal substrate. Grains can either be randomly oriented or they can exhibit a preferential orientation with respect to the substrate. Grains that exhibit a similar preferential orientation are said to belong to a specific texture component and they can exhibit one of three types of texture: (1) epitaxy or in-plane texture, where the orientation of the grains is uniquely defined with respect to the substrate and only one single orientation (and the symmetrically equivalent orientations) occurs; (2) fiber, where one of the (hkl) planes is oriented (nearly) parallel to the film/substrate interface and different grains exhibit a different rotation around the axis perpendicular to the interface, i.e., the fiber axis; (3) axiotaxy, where a specific plane in the film is aligned to a specific plane in the substrate which is not parallel to the interface, resulting in an off-normal fiber texture. More detail on these texture types, especially the axiotaxy texture, will be provided in Section III.

Because these silicides and germanides are important materials in microelectronics, a thorough understanding of their properties has direct impact on device fabrication and performance. As will be discussed in this review, texture can have a profound influence on the formation and stability of these silicide/germanide contacts as it controls the type and orientation of grain boundaries (affecting diffusion and agglomeration) and the interface energy (affecting nucleation during the solid-state reaction). Furthermore, texture also has an impact on the electrical characteristics of the contact as the orientation and size of individual grains will influence functional properties such as contact resistance and sheet resistance and will induce local variations in strain and Schottky barrier height (SBH). Of course, the ultimate goal is to be able to predict the texture that will develop for a certain set of experimental parameters (e.g., thin film material, annealing conditions, substrate properties such as doping and cleaning methods, etc.). However, results obtained so far in this area simply do not allow yet to construct such predictive theories. Therefore, studies concerning attempts to influence texture formation in specific cases will be discussed at length in this review, but no general theories for texture prediction will be put forward.

The plan for this review is as follows. In Section II, we will briefly discuss the most important experimental techniques for measuring texture that have been used in literature over the past decades, along with their advantages and disadvantages. In Sections III to V, we aim to provide a comprehensive overview of literature reports in the field over the past few decades. We start off with a general overview of the types of texture observed in different silicide and germanide materials in Section III, with a focus on the concept of axiotaxy. Section IV covers studies probing the influence of texture on the silicide/germanide thin film properties such as formation characteristics, phase stability, agglomeration behavior, and electrical properties. Finally, in Section V, we discuss different factors that can influence the texture of the final silicide phase during its formation and that hence can be exploited in efforts to control the texture of the contacting material.

\section{MEASURING TEXTURE IN THIN SILICIDE/ GERMANIDE FILMS}

In this section, we provide a brief introduction of the two best suited techniques to study texture in thin film silicides/germanides, i.e., X-ray pole figures and Electron Backscatter Diffraction (EBSD). It must be noted though that these techniques are not the ones that were adopted in the early days of texture research on thin silicide films. As will be discussed in Section III A, during the 1970s and 1980 s, research efforts were mainly focussed on the growth of epitaxial silicide films. In order to probe the epitaxial quality of the grown films, two techniques were mainly used: ion channeling and transmission electron microscopy.

Ion channeling is performed using Rutherford Backscattering Spectroscopy, which is why this technique is often referred to as $R B S$-channeling. In such an experiment, the sample is aligned with a major crystallographic direction directed along the incoming ion beam. The reduced yield of backscattered ions is then used as a measure for the epitaxial quality of the film. For a more detailed explanation of the technique, the interested reader is referred to Ref. 27.

Transmission Electron Microscopy or TEM is a technique in which electrons are transmitted through very thin samples that are either prepared in plan view (electrons are incident perpendicular to the silicide film surface) or in cross section (electrons are incident parallel to the interface). The transmitted electrons can be used for standard high resolution imaging or for diffraction to determine the structure and orientation of crystallites in the film. The high spatial resolution of TEM based techniques is also their major drawback when applied to texture studies: because only a limited number of grains can be measured within a reasonable time, a material with a complex texture will only be partially characterized. While this technique is still heavily used today for microstructure characterization, the statistical reliability of claims regarding texture based solely on TEM measurements is low and complementary techniques like EBSD and X-ray pole figure measurements are required.

\section{A. X-ray diffraction-Pole figures}

$\mathrm{X}$-ray diffraction is a widely adopted technique for studying the internal (crystalline) structure of materials. The most used geometry for X-ray diffraction characterization is the so called $\theta / 2 \theta$-measurement in which an $\mathrm{X}$-ray beam 
impinges on a sample at an incoming angle $\theta$ with respect to the sample surface and a detector is positioned at an exit angle of $2 \theta$ with respect to the direction of the incoming beam. In this configuration, diffraction can be observed from a set of planes with interplanar spacing $d_{h k l}$ oriented parallel to the sample surface if the Bragg condition $n \lambda=$ $2 d_{h k l} \sin (\theta)$ is fulfilled (with $\lambda$ the wavelength of the X-ray beam). The resulting diffraction pattern is a characteristic fingerprint of interplanar distances present in a specific crystalline material. As such, it provides an ideal basis for material identification. In thin film silicide/germanide research, this $\theta / 2 \theta$-scan has become a run-of-the-mill technique for identifying the specific silicide/germanide phase(s) present in a film.

The main disadvantage of such a standard $\theta / 2 \theta$ scan is the aforementioned fact that it is only sensitive to sets of lattice planes oriented parallel to the sample surface (thus parallel to the substrate). For a film consisting of randomly oriented grains, this characterization is sufficient, since different lattice planes will be parallel to the substrate in at least some of the grains. However, for a textured film where the grains have a strong preferential alignment with respect to the substrate, one can imagine that certain lattice plane sets will never be aligned parallel to the surface and thus will never meet the diffraction criterion. As a result, in a $\theta / 2 \theta$ scan of a textured film, certain diffraction peaks will be absent, making phase identification considerably harder. In this case, pole figure measurements are necessary to uniquely identify the observed phase and its texture.

A geometry commonly used to measure pole figures using X-ray diffraction is based on the Schulz method, ${ }^{28}$ introduced in 1949 and embraced for texture studies in the field of metallurgy. Here, the sample is placed on a fourcircle diffractometer which allows to tilt ( $\chi$ angle) and rotate ( $\phi$ angle) it and to choose its inclination with respect to the incoming X-ray beam ( $\theta$ angle). The detector is then positioned at an angle $2 \theta$ with respect to the incoming X-ray beam in order to fulfill the Bragg diffraction condition. A schematic of this setup can be found in Fig. 2(a). By using a point detector and fixing the $\theta$ angle, one measures the diffracted intensity of a specific family of crystal planes. Measuring this diffracted intensity while tilting the sample around $\chi$ and rotating it around $\phi$ allows one to measure diffraction for the chosen family of lattice planes for every possible grain orientation, not just the one where the planes happen to be parallel to the sample surface. The result of such a measurement can be understood by imagining the sample placed at the centre of a hemisphere on which one marks for each grain the intersection between the normal to the chosen family $\{h k l\}$ of lattice planes (also called the pole of the plane family) and the sphere (see Fig. 2(b) as an illustration). The final ( $h k l)$ pole figure is then obtained by projecting the density of marks on the sphere onto a planar surface (see Fig. 2(c)) and thus depicts the statistical angular distribution of the direction of the normal to this plane family. The spherical coordinates $(\chi, \phi)$ of the poles now become polar coordinates, with $\chi$ the radial distance and $\phi$ the polar angle. For a more detailed description on pole figures, the interested reader is referred to Refs. 24-26.

In order to relate the directions of the film poles observed in a pole figure to directions of substrate poles and thus determine the orientation of a grain with respect to the substrate, a frame of reference is needed. This frame of reference is established by aligning the sample in order to locate poles for low-index substrate planes at specific (simple) $(\chi, \phi)$ coordinates. The most frequently used reference frames in literature for (001), (110), and (111) Si or Ge substrates are depicted in Fig. 3. Unless stated otherwise, the pole figures shown in this review are measured using these reference frames.

An early use of this technique in texture research on thin film silicides dates back to 1992, when Bulle-Lieuwma et al. used lab-based X-ray diffraction to measure pole figures for texture studies of $\mathrm{CoSi}_{2}$ films $^{29}$ (see Fig. 4(a)). In 2002,

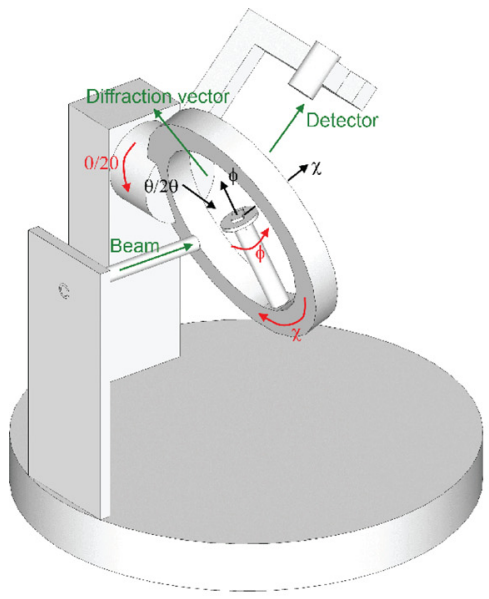

(a)

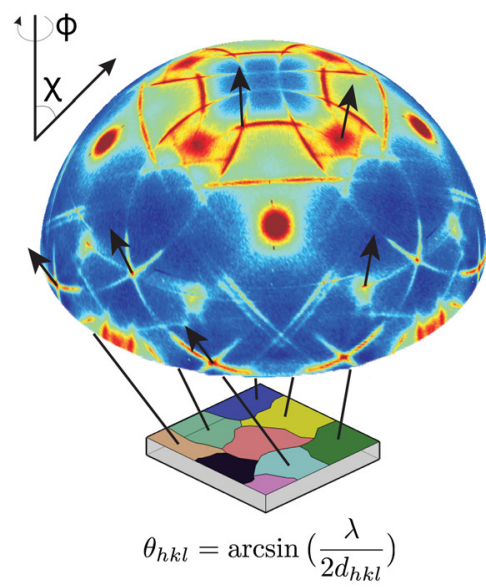

(b)

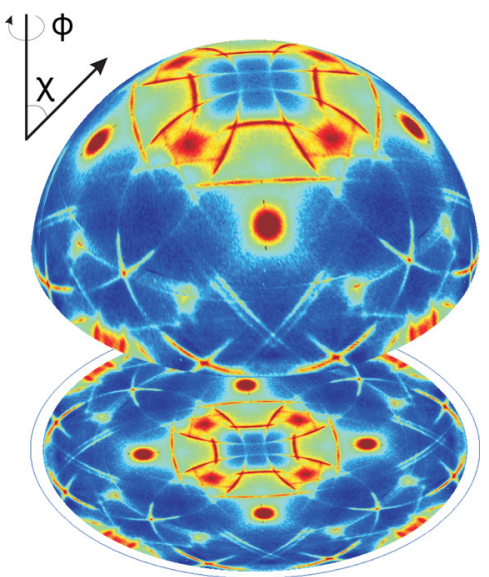

(c)

FIG. 2. (a) Illustration of a four-circle diffractometer used in the Schulz method. The different rotation axes ( $\phi, \chi$, and $\theta)$ are depicted along with the corresponding rotation directions. (b) Theoretical construction of a pole figure. The intensity distribution on the imaginary hemisphere is created by marking the intersection of the normals of the diffracting planes (those that fulfill the Bragg condition for the chosen d-spacing) with the hemisphere for each grain in the film. (c) Projection of the intensity distribution on the hemisphere onto a planar surface results in a pole figure for a specific family $\{h k l\}$ of lattice planes. The data used in this illustration are a (112) pole figure of a NiSi film on $\mathrm{Si}(001)$. 


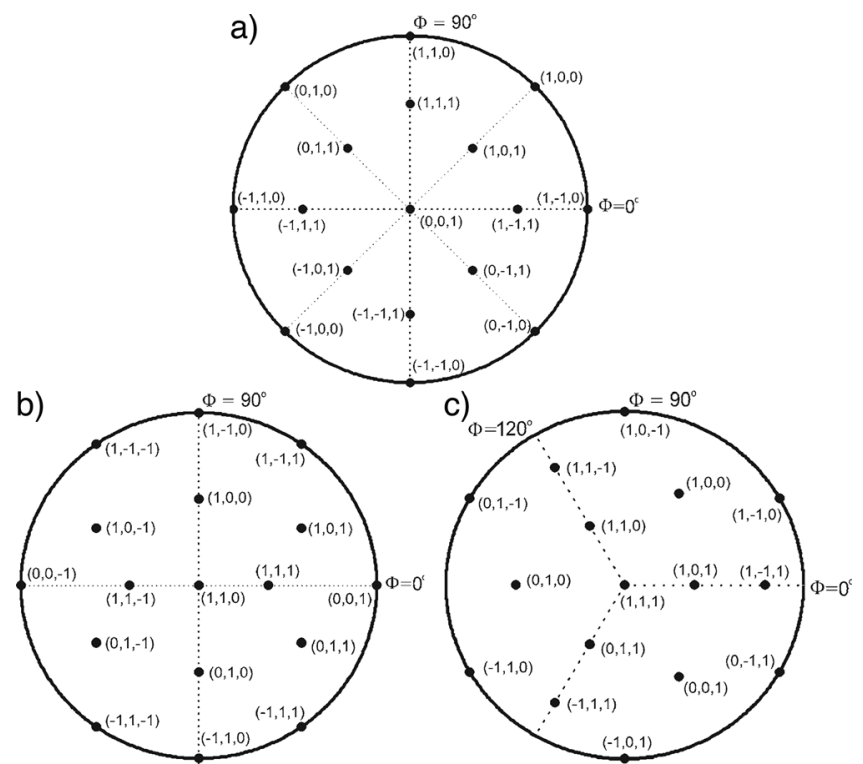

FIG. 3. Reference frames for the three types of $\mathrm{Si}$ or Ge substrates: (a) (001), (b) (110), and (c) (111). The figures show the $(\chi, \phi)$ locations of the low-index poles of the substrate.

Özcan et al..$^{30}$ used synchrotron radiation to measure pole figures on $\mathrm{TiSi}_{2}$ films, greatly reducing the measurement time for a single measurement compared to a lab-based X-ray setup (see Fig. 4(b)). Further improvements to the technique eventually led to the discovery of axiotaxy in NiSi films ${ }^{31}$ (see Fig. 4(c)), which will be discussed in Section III C. Over the past decade, the texture of several thin film silicides and germanides has been investigated
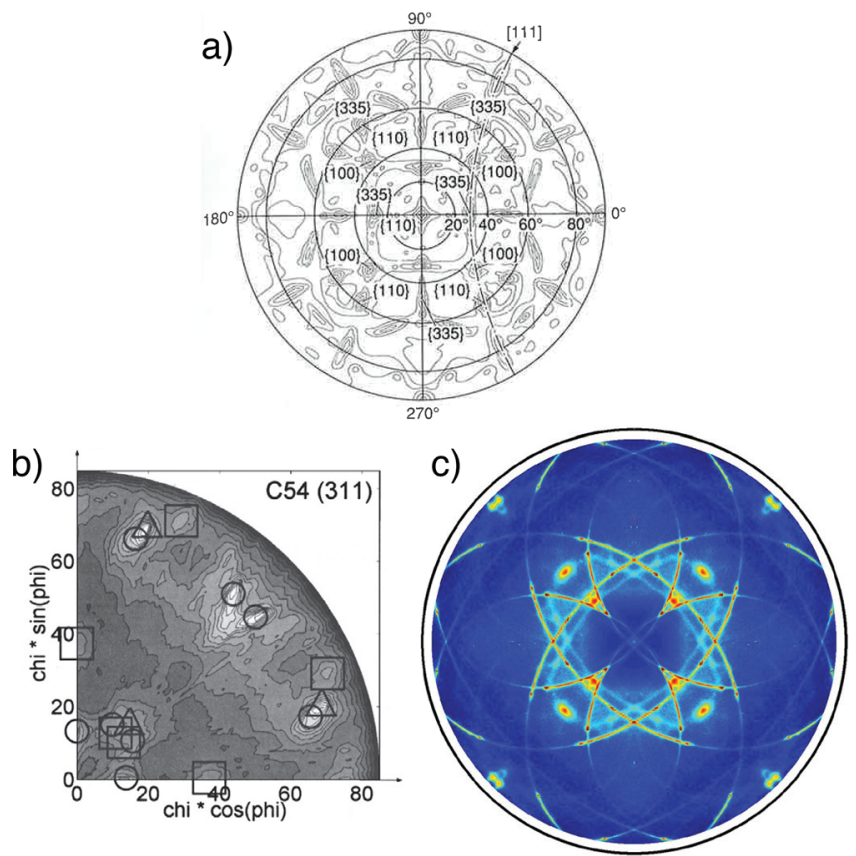

FIG. 4. (a) $\mathrm{CoSi}_{2}$ (110) pole figure recorded using a lab-based X-ray diffraction setup (reprinted with permission from J. Appl. Phys. 71, 2211 (1992). Copyright 1992 AIP Publishing LLC.). (b) C54-TiSi 2(311) pole figure (reprinted with permission from J. Appl. Phys. 92, 5011 (2002). Copyright 2002 AIP Publishing LLC.) and (c) $\mathrm{NiSi}(002) /(011)$ pole figure measured using a synchrotron based diffraction setup. using these high-resolution synchrotron based pole figure measurements (see Section III B). It must be noted that with the term high-resolution we want to emphasize the much improved resolution compared to pole figures measured on thin silicide/germanide films using a lab-based X-ray source. The typical resolution in such a synchrotron based pole figure measurement is $1^{\circ}$ in $\chi$, while the step size in $\phi$ is usually dynamically adapted during the measurement in order to obtain a uniform measurement grid.

A pole figure measured using the Schulz method described above only provides information for one specific d-spacing, i.e., usually a single lattice plane. Because the orientation of one plane does not fix the orientation of a grain, multiple pole figures for multiple families of crystal planes have to be measured in order to obtain complete texture information. As the acquisition time for a single pole figure measurement on a thin silicide film typically requires a few hours, this process becomes very time-consuming. A solution is to use a linear or area detector covering a range of $2 \theta$ angles (or d-spacings) instead of a point detector, which only records diffraction at a single $2 \theta$ angle. In this way, several pole figures for different d-spacings can be measured simultaneously in a single measurement. Furthermore, the use of linear/area detectors also allows for the study of films containing unknown phases for which the $2 \theta$ positions of diffraction peaks are not known prior to the measurement. This idea of simultaneously measuring pole figures for different $2 \theta$ angles was introduced in the field of metallurgy in the $1980 \mathrm{~s},{ }^{32}$ but was only recently adopted in the field of thin silicide/germanide films. ${ }^{33-36}$ Technical and mathematical details on using linear or area detectors for pole figure measurements can be found in Refs. 37 and 38.

\section{B. Electron backscattered diffraction (EBSD)}

Over the past decades, research fields such as metallurgy, ceramics, and geology have exploited Electron Backscattered Diffraction (EBSD) which has become a standard technique to study different structural material properties such as grain size, grain boundary types, and crystallographic texture. In this paragraph, the applicability of this technique for texture studies in thin film silicides/germanides will be discussed.

A typical EBSD setup (see Fig. 5(a)) is integrated in a Scanning Electron Microscope (SEM) and consists of a dedicated sample holder with a high tilt angle and an EBSD detector (usually a combination of a phosphor screen located in front of a CCD camera). The interaction between the incoming electron beam and the nuclei in the sample results in the generation of backscattered electrons. Part of these backscattered electrons will eventually leave the sample after being diffracted by the local crystal structure on their way out. These electrons will generate an Electron Backscattered Diffraction Pattern (EBSP) which displays a collection of bands referred to as Kikuchi lines (see top part of Fig. 5(b)). Analysis of the generated EBSP from a single grain is done by a procedure called indexing and provides information on the crystal structure and its orientation with respect to the substrate and the neighboring grains. For a detailed 


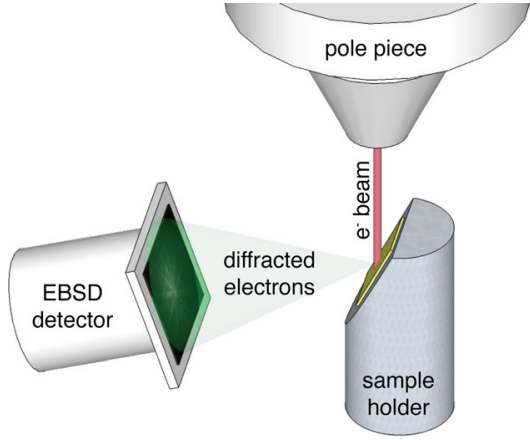

(a)

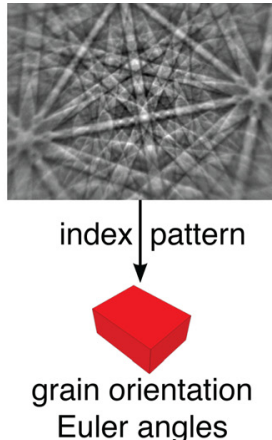

(b)

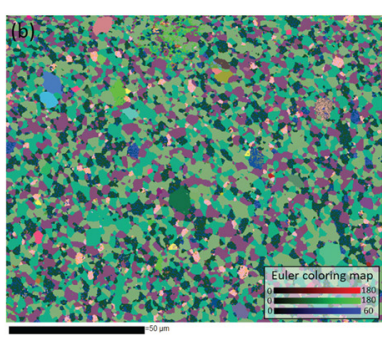

(c)
FIG. 5. (a) Schematic of a typical EBSD setup inside a SEM (Scanning Electron Microscope). (b) Example of a recorded electron backscattered pattern (EBSP) showing the Kikuchi bands. Indexing such a pattern provides information on the local crystal phase and orientation. (c) EBSD map carried out on $\theta$-nickel-silicide over a rectangular measurement grid. $^{39}$ Different crystal orientations are given a unique color. In this way, individual grains and grain boundaries become visible. description of the generation and indexing of these EBSP's, the reader is referred to Ref. 40 .

While local determination of crystal structure and orientation can definitely prove very useful, the power of the EBSD technique resides mostly in its ability to perform measurements on a large grid. By measuring and indexing an EBSP on every grid point (which can be done automatically with dedicated indexing software), one can identify areas with the same crystal orientation. This large dataset provides a way to visualize the individual grains in the film along with their orientation and hence allows to study microstructural features such as grain sizes, grain boundaries, and texture. Furthermore, the local orientation information of a single grain and its neighbors provided by EBSD can be very useful in understanding macroscopically observed film properties (stress, contact resistance, etc.), as some silicides/germanides are known to be highly anisotropic (e.g., NiSi). An example of such an EBSD map on $\theta$-nickel-silicide performed on a $50 \mu \mathrm{m} \times 50 \mu \mathrm{m}$ rectangular grid can be seen in Fig. 5(c), where each color corresponds to a specific crystal orientation.

The application of EBSD in the field of texture studies on thin silicide/germanide films was first reported in 2007 by De Keyser et al., ${ }^{41}$ who used the technique to study the texture of NiSi films on $\mathrm{Si}(001)$. Doing so, they were able to confirm the texture components that were previously reported in NiSi films using X-ray pole figure measurements, but EBSD also allowed them to directly calculate volume fractions for the different texture components, which is indirect and much more involved to extract from X-ray pole figure measurements.

Although EBSD appears ideal to study texture in thin film silicides/germanides, some important limitations must be recognized. ${ }^{39,41}$ First of all, as grain sizes in very thin films can be quite small, the spatial resolution of a typical EBSD setup (usually a few tens of nanometers) may be inadequate to resolve individual grains. This leads to poorer quality and more difficult indexing of the recorded EBSD patterns as different grains (and thus different crystal orientations) will contribute to a single measured pattern. Second, interaction between the electron beam and the sample surface will lead to decomposition of residual hydrocarbons in the SEM chamber, resulting in a trail of carbon deposited on the sample. As an EBSD pattern is a diffraction pattern, any surface contamination will negatively influence the pattern's quality. One can circumvent this second issue by working with a (very expensive) UHV system. Ultimately, a trade-off has to be made between increasing the measurement time to increase the number of collected electrons in an effort to improve the pattern quality and decreasing it to lower surface contamination. In addition, lower quality EBSP's were also observed for alloyed silicides like $\mathrm{Ni}_{1-x} \mathrm{Pt}_{x} \mathrm{Si}$, possibly due to the lower crystallinity of these materials. ${ }^{41}$ Nonetheless, EBSD has been successfully applied to a few thin film silicide and germanide materials over the past years. ${ }^{36,41-46}$

\section{OVERVIEW OF TEXTURE IN THIN SILICIDE/ GERMANIDE FILMS}

In this section, we survey the earlier research on texture in thin silicide and germanide films. First, a short historical overview will be given covering the focus on epitaxial silicides during the 1970s, 1980s, and 1990s up until the advent of more detailed studies on polycrystalline silicide and germanide films using high-resolution synchrotron based pole figure measurements. Second, we will focus on the driving forces for texture development and the phenomenon of axiotaxy, a new type of texture in thin silicide/germanide films that was first reported in 2003. ${ }^{31}$ Finally, we will briefly discuss the texture observed in the three silicides that have been technologically most relevant over the past two decades: C54-TiSi ${ }_{2}, \mathrm{CoSi}_{2}$, and NiSi. As a summary, Table I gives an overview of texture studies performed on a selection of silicide and germanide thin film materials. For each material, the observed texture components are provided along with key references, categorized by the measurement technique(s) used in the studies.

\section{A. History of texture studies in thin silicide/germanide films}

Interest in the texture of thin film silicides increased through the 1970s when it was discovered that certain silicides-which were investigated at that time for use as ohmic contacts, Schottky barriers, gate electrodes, and interconnects in microelectronic devices-grew epitaxially on single crystal silicon substrates. By the end of the 1970s, there were four generally known epitaxial silicides, i.e., $\mathrm{NiSi}_{2},{ }^{52}$ $\mathrm{CoSi}_{2},{ }^{54} \mathrm{PtSi}^{73}$ and $\mathrm{Pd}_{2} \mathrm{Si}^{70,71}$ for which the research on their epitaxial growth was reviewed by Tung et al. in $1982 .{ }^{89}$ In the following years, the focus in texture research on silicide films remained on the epitaxial growth as it was 
TABLE I. Overview of published studies concerning texture in thin silicide/germanide films on single crystal Si or Ge substrates. For each material, the types of texture that have been observed in different studies are given, along with references to the studies grouped by the experimental technique that was adopted.

\begin{tabular}{|c|c|c|c|c|c|}
\hline \multirow[b]{2}{*}{ Material } & \multirow[b]{2}{*}{ Observed texture } & \multicolumn{4}{|c|}{ References per measurement technique } \\
\hline & & RBS-C ${ }^{\mathrm{a}}$ & $\mathrm{ED}^{\mathrm{b}}$ & $\mathrm{XRD}^{\mathrm{c}}$ & EBSD $^{\mathrm{d}}$ \\
\hline $\mathrm{NiSi}$ & epi, axio, fiber, transrot & & $47-49$ & 31 and $49-51$ & 41 \\
\hline $\mathrm{NiSi}_{2}$ & epi & $52-54$ & 47 and $52-54$ & 52 & \\
\hline$\delta-\mathrm{Ni}_{2} \mathrm{Si} / \theta-\mathrm{Ni}_{2} \mathrm{Si}$ & epi, fiber, transrot & & & 33,35 , and 46 & 46 \\
\hline $\mathrm{TiSi}_{2}(\mathrm{C} 49 / \mathrm{C} 54)$ & epi, axio & & $55-61$ & 30 and 59-63 & \\
\hline $\mathrm{CoSi}_{2}$ & epi, axio & 54 and 64 & $29,54,64$, and 65 & $29,65-67$ & \\
\hline$\alpha-\mathrm{FeSi}_{2}$ & epi, axio & & & 68 and 69 & \\
\hline $\mathrm{Pd}_{2} \mathrm{Si}$ & epi & 70 and 54 & 54 and $70-72$ & & \\
\hline $\mathrm{PtSi} / \mathrm{Pt}_{2} \mathrm{Si}$ & epi & & $73-76$ & & \\
\hline $\mathrm{CrSi}_{2}$ & epi, axio & & 77 & 77 & \\
\hline Rare-earth silicides/germanides & epi & 78 & 79 and 78 & 78,80, and 81 & \\
\hline $\mathrm{NiGe}$ & epi, axio & & & 82,83 & \\
\hline $\mathrm{Co}_{5} \mathrm{Ge}_{7}$ & epi, axio & 84 & 84 and 85 & 42 & 42 \\
\hline $\mathrm{CoGe}_{2}$ & epi, axio & 84 & 84 & 42 & 42 \\
\hline $\mathrm{Pd}_{2} \mathrm{Ge} / \mathrm{PdGe}$ & epi, axio, fiber & & 86 & 86 and 87 & \\
\hline Pt-germanides & epi & & 88 & & \\
\hline
\end{tabular}

${ }^{\mathrm{a}}$ RBS-channeling.

${ }^{\mathrm{b}}$ Electron diffraction techniques.

${ }^{c} \mathrm{X}$-ray diffraction.

${ }^{\mathrm{d}}$ Electron backscatter diffraction.

believed that using epitaxial silicides in microelectronics would lead to enhanced electrical contacts due to the excellent quality of the epi-silicide/silicon interface. ${ }^{90}$

In general, epitaxy can be expected when the silicide and the silicon substrate have matching periodicity in the plane of the interface. For an epitaxial alignment to be observed, it is generally assumed that the mismatch between the lattices must be below $2 \%$ in the plane of the interface. Fig. 6 shows an overview of different silicide phases along with their lattice mismatch to $\mathrm{Si}(001), \mathrm{Si}(110)$, and $\mathrm{Si}(111)$ and their unit cell area. From the substantial amount of silicide phases visible in this figure, only the bottom left part (with $\mathrm{NiSi}_{2}$ and $\mathrm{CoSi}_{2}$ as the most important ones) will actually form epitaxial silicides through a simple solid-state reaction between a thin metal film and a silicon substrate. By the early 1990s, a lot of effort had gone into improving existing and developing novel deposition techniques in order to grow silicides, -at least partially, epitaxial on silicon. ${ }^{90}$ The most important techniques are listed below:

- Solid Phase Reaction (SPR)—A thin metal film is deposited on the silicon substrate, usually through evaporation techniques, followed by a thermal anneal to form the desired epitaxial silicide phase.

- Co-deposition and Reactive Deposition Epitaxy (RDE) Co-deposition of metal and Si vapor onto a heated substrate (often in an MBE system) results in the deposition of silicide layers. By controlling the metal/Si flux and the temperature, several authors have reported the formation of nucleation-controlled disilicides at temperatures significantly below their standard nucleation temperature. ${ }^{14,91}$

- Templated MBE-A thin epitaxial silicide film is grown using SPR which then serves as a template for continued growth through $\mathrm{MBE}$. $^{92}$
- Oxide/Titanium Mediated Epitaxy (OME/TIME)Dedicated technique for growing epitaxial $\mathrm{CoSi}_{2}$ films, introduced by Dass ${ }^{93}$ (TIME, 1991) and Tung ${ }^{94}$ (OME, 1996). Epitaxial $\mathrm{CoSi}_{2}$ layers are grown by introducing a very thin interlayer of Titanium (TIME) or silicon oxide (OME) between the silicon substrate and the Co film. Annealing this stack between 500 and $700{ }^{\circ} \mathrm{C}$ leads to the growth of epitaxial $\mathrm{CoSi}_{2}$ films. It is believed that the interlayer acts as a diffusion barrier, limiting the flux of Co towards the Si substrate and in this way promoting epitaxy. The OME technique turned out to deliver the best quality of epitaxial $\mathrm{CoSi}_{2}$ films. ${ }^{95}$ Later, it was also observed that interlayers of other metals like $\mathrm{Cr}$, Mo, Ta, and $\mathrm{W}$ can lead to epitaxial $\mathrm{CoSi}_{2}$ if the thickness of the interlayer is carefully chosen. ${ }^{96,97}$

- Ion beam synthesis (sometimes referred to as mesotaxy)A technique to form epitaxial silicides buried in the silicon substrate. First, the buried silicide is formed through ion implantation of the transition metal (e.g., Co) into the substrate at an elevated temperature $\left(300-400^{\circ} \mathrm{C}\right)$. Next, an anneal at higher temperature results in an epitaxial buried silicide. The technique was introduced by White et al. in $1986 .^{98}$ The interested reader is referred to Ref. 99 for a detailed review on the technique.

- Allotaxy-An alternative technique for the growth of epitaxial buried silicides by using standard Molecular Beam Epitaxy instead of ion implantation, introduced by Mantl et al. in 1992. ${ }^{100}$ The technique uses a sequence of MBE steps to grow epitaxial silicide precipitates in a single crystalline Si matrix on top of the Si substrate. High temperature annealing then forms the buried epitaxial layer out of the precipitates. A more detailed description can be found in Ref. 101. 


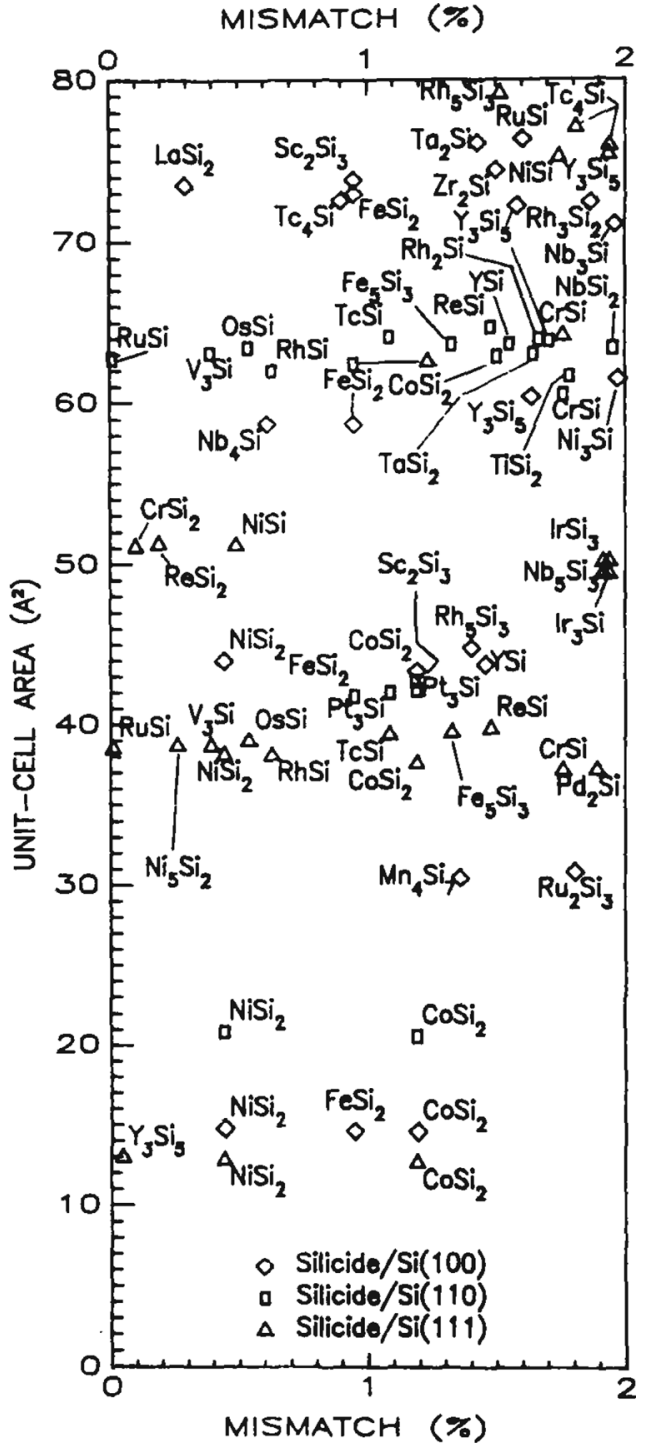

FIG. 6. Overview of transition-metal silicides as a function of the lattice mismatch between film and substrate and the unit-cell-area of the matching area between film and substrate for $\mathrm{Si}(100), \mathrm{Si}(110)$, and $\mathrm{Si}(111)$ substrates. Figure reprinted with permission from J. Appl. Phys. 57, 600 (1985). Copyright 1985 AIP Publishing LLC.

As texture research on thin film silicides at that time was mainly focussed on the growth of epitaxial films, little attention was given to the texture of silicides that were not suited for epitaxial growth, i.e., the majority of silicides. Because a solid-state reaction driven growth of these nonepitaxial films mostly leads to small-grained polycrystalline films, they were assumed to be randomly textured. During the 1990s, it became increasingly clear that the use of epitaxial silicides in commercial microelectronic devices would be very difficult. As the SALICIDE process became the industry's technique of choice for the formation of silicide contacts on CMOS devices, studies started to emerge focussing on the texture of these polycrystalline, technologically relevant silicide thin films $\left(\mathrm{CoSi}_{2}, \mathrm{TiSi}_{2}\right){ }^{29,59}$ It was readily observed that these polycrystalline films display complex texture. This is illustrated by the Bragg-Brentano XRD spectra of NiSi on different Si substrates shown in Fig. 7, where

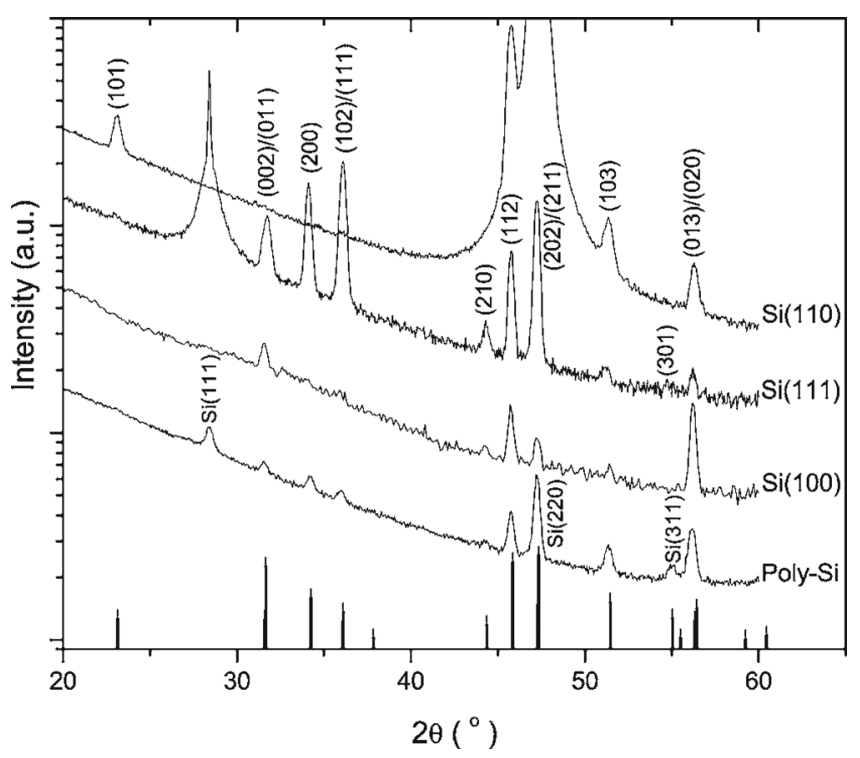

FIG. 7. $\theta / 2 \theta$ XRD measurements for a thin NiSi film on $\mathrm{Si}(001), \mathrm{Si}(110)$, $\mathrm{Si}(111)$ and poly-crystalline $\mathrm{Si}$ (poly-Si). The indexing of the peaks is done based on orthorhombic NiSi (JCPDS 73-1843). The theoretically expected relative peak intensities for a randomly oriented NiSi powder are visualized by the series of vertical lines at the bottom of the figure. The different relative intensities of the peaks for the single crystalline substrates are indicative of a non-random texture on these substrates. Figure reprinted with permission from J. Appl. Phys. 103, 113526 (2008). Copyright 2008 AIP Publishing LLC.

it can be observed that the films are not randomly textured, as the relative peak intensities differ greatly from what is expected for a random powder of NiSi (indicated by the vertical lines at the bottom of the figure). The use of highresolution synchrotron based pole figure measurements in the early 2000s really accelerated the study of texture in these thin silicide films.

And what about germanides? Because silicon had been the dominant material in the semiconductor industry for more than three decades, applications for germanide films were far less common than for silicide films. As a result, most early studies focus on silicide materials. However, since the early 2000s, germanium has been reintroduced in the semiconductor industry through the use of $\mathrm{Si}_{1-x} \mathrm{Ge}_{x}$ either to stress the channel of the transistor or more recently even as a replacement material for the channel altogether. For the future, researchers have been investigating the use of pure $\mathrm{Ge}$ as an alternative channel material because of its superior carrier mobilities compared to $\mathrm{Si}^{22}$ In this context, germanides are now being investigated as possible contacting materials for these Ge based devices, resulting in a considerable increase in germanide research during the past decade. ${ }^{23}$ Nonetheless, literature on texture in these germanide materials is still scarce. There are a few early studies on the texture of $\mathrm{Co}_{5} \mathrm{Ge}_{7}$ on $\mathrm{Ge}(001)$ and (111), ${ }^{84,85} \mathrm{CoGe}_{2}$ on $\mathrm{Ge}(111),{ }^{84} \mathrm{Pd}_{2} \mathrm{Ge}$ on $\mathrm{Ge}(111),{ }^{86} \mathrm{Pt}_{2} \mathrm{Ge}, \mathrm{PtGe}$ and $\mathrm{PtGe}_{2}$ on $\mathrm{Ge}(111),{ }^{88}$ and NiGe on Ge(111). ${ }^{102}$ Similar to silicides, the introduction of high resolution pole figure measurements has allowed for a more detailed assessment of the texture in a few thin film germanides over the past decade. ${ }^{42,83,87}$ 


\section{B. Pole figure based texture studies}

Figure 8 shows a collection of high-resolution synchrotron based X-ray pole figures measured over the past decade on a variety of silicide and germanide thin films grown on different substrate types. Complex patterns consisting of a combination of lines and spots can be observed, indicating complex texturing of these films. The different texture components listed in the introduction that can occur in thin silicide/germanide films all produce specific features in a pole figure. This makes pole figure measurements an ideal technique to identify which texture components are present in a thin silicide/germanide film. The patterns that are generated by the different texture components are briefly discussed below:

- Random texture-Random (or absence of) texture corresponds to a uniform distribution of grain orientations, i.e., no preferential orientation is present in the film. This means that the pole figure for a specific $\{h k l\}$ family of crystal planes will look featureless since every orientation of the $\{h k l\}$ pole will be equally probable. This is illustrated in Fig. 9(a).

- Fiber-A fiber texture is characterized by the constraint that one plane normal in all of the grains is perpendicular to the interface (this direction is called the fiber-axis). This will translate into a single spot in the center of the pole figure for that specific (hkl) plane. Due to the rotational degree of freedom around the fiber axis for the grains in a fiber texture, the plane normal of any other crystal plane will describe a circle around the fiber axis. This translates into a centered ring on the pole figure (see Fig. 9(b)). Since a pole figure measurement for a specific (hkl) plane (thus for a specific d-spacing) also records diffracted intensity for all the symmetrically equivalent planes, as well as for other planes that happen to share the same d-spacing, multiple centered rings can appear on a single pole figure.
- Axiotaxy-Axiotaxy can be understood as an off-normal fiber texture (i.e., the fiber axis is not perpendicular to the interface). As such, this results in pole figures displaying circles around off-center positions on the pole figure (see Fig. 9(c)). A detailed description of this texture component, first measured in 2003 using high-resolution X-ray pole figures, ${ }^{31}$ will be given in Sec. III C.

- Epitaxy-All grains belonging to a specific epitaxial texture component have the same orientation. This means that for any crystallographic plane, the plane normal will have a unique orientation. This will lead to a set of well-defined spots on the pole figures (due to symmetrically equivalent planes being recorded too). This is illustrated in Fig. 9(d).

As can be seen from Table I, a combination of different texture components has been observed in many silicide/germanide thin films grown by solid-state reactions. These observations have only been possible, thanks to the development of the synchrotron based X-ray pole figure technique introduced by Özcan et al. in 2002 to study the texture of C54-TiSi ${ }_{2}{ }^{30}$ Only a year later, the use of high-resolution synchrotron based pole figures resulted in the discovery of axiotaxy. ${ }^{31}$ In the following years, detailed texture studies of different silicide and germanide materials were carried out using this technique, revealing axiotaxy in a considerable number of silicide/germanide materials (see Table I). More recently, the use of a linear or area detector instead of a point detector $^{37,38}$ and the introduction of EBSD as a complementary technique ${ }^{41}$ have enabled the ability to perform unambiguous phase identification of highly textured transient phases that form during the formation of $\mathrm{NiSi}^{33,35}$ or $\mathrm{NiGe}^{103}$ (see Section IV A). In the near future, area detectors will be routinely used to record texture information in situ, enabling the study of texture evolution during silicide/germanide formation. This is possible as using an area detector effectively shortens the time needed for a pole figure
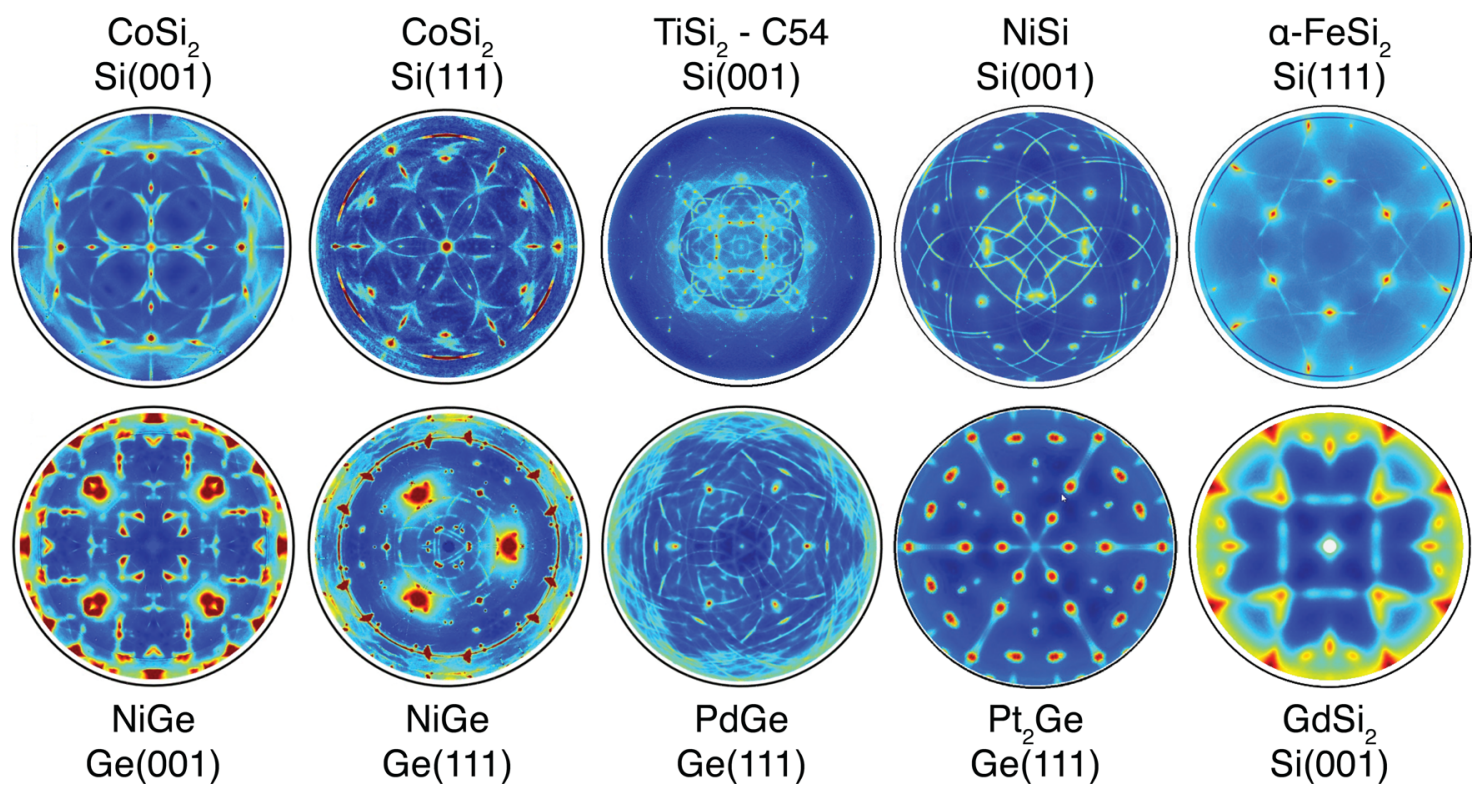

FIG. 8. Collection of pole figures measured on different silicide and germanide materials grown on different substrate orientations. The pole figures were measured during the past decade using the high-resolution synchrotron based approach. Complex patterns of lines and spots can be observed, indicating complex non-random texturing in these films. 


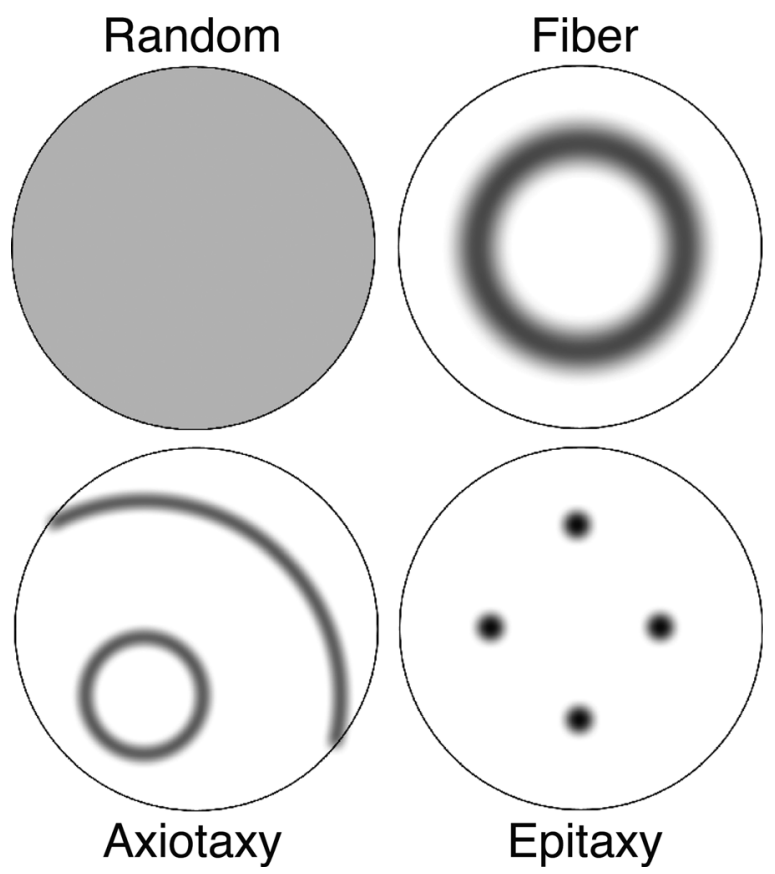

FIG. 9. Sketches of pole figure features generated by the four possible texture components: random, fiber, axiotaxy, and epitaxy.

measurement from hours to minutes, allowing one to measure a pole figure every few minutes while heating the sample at a steady heating rate. ${ }^{38}$

\section{Driving forces for texture selection}

In general, the preferential orientation of grains in a thin film with respect to a single crystal substrate is driven by a minimization of the interface energy. Grains with different orientations will exhibit different interface energies as the bonding across the interface will be different. Thus, the lowest interface energies are achieved by optimizing this bonding across the interface between the film grain and the single crystal substrate.

\section{Importance of periodicity}

First, we consider the case of a perfectly flat interface between the film grain and the substrate. As both the film grain and the substrate have a periodic crystal structure, bonding can only be systematically optimized along a certain direction within the plane of the interface if the interface structure is periodic. This periodic interface structure then amounts to a "matching" of the grain lattice and the substrate lattice along that direction within the plane of the interface. Optimization of the bonding may then occur through interface reconstruction, whereby the atoms in the first few atomic layers near the interface are re-arranged, analogous to surface reconstruction at the crystal/vacuum interface.

For a grain with an epitaxial orientation, this means that a periodic interface structure and thus a match within the plane of the interface must be achieved in two independent directions. When the film lattice and the substrate lattice have a similar crystal structure and the mismatch between the lattice constants of film and substrate is small, this can be easily achieved (e.g., $\mathrm{NiSi}_{2}$ on $\mathrm{Si}$ ). In the situation where the film and substrate material have a different crystal structure, such a 2D interface match is much less likely. In this case, a reduced interface energy can still be achieved by a match between a low-index plane from the grain and a low-index plane from the substrate that arrive at the interface, effectively creating a one-dimensional periodicity within the plane of the interface. The simplest way in which this can be accomplished is when the film and substrate lattice plane have a (nearly) identical d-spacing. In this case, the film and substrate planes will be parallel and aligned across the interface (see Fig. 10, left column). This kind of preferential grain orientation is called "axiotaxy" and was first observed in thin NiSi films on $\mathrm{Si}(001)$ by Detavernier et al. in $2003 .{ }^{31}$ Because of the one-dimensional periodic interface structure achieved by plane alignment, the grains are left with one rotational degree of freedom around the normal to the matched plane in the film. As mentioned earlier, this causes circles on a pole figure which are centered around the location of the normal to the matched plane, called the "offnormal fiber axis" or "axiotaxy axis" (see Fig. 9).

In principle, such a one-dimensional interface structure by plane matching can also be achieved for film and substrate planes with a different d-spacing by tilting the grain (over an angle $\Delta \chi$ ) in such a way that the d-spacing projected onto the interface plane becomes equal for the film and lattice plane (see Fig. 10, right column). As such, axiotaxy could be expected to form very easily. However, texture studies on different silicides and germanides (see Table I) have shown that when axiotaxy is observed, the circles on the pole figures are always centered around a point that coincides or nearly coincides with the location of the pole of the low-index substrate plane that is matched with a low-index film plane, meaning that the tilt angle $\Delta \chi$ must be small for axiotaxy to be observed. This suggests that axiotaxy only occurs if the grain can be oriented in such a way that a lowindex film plane and a low-index substrate plane are (nearly) aligned across the interface.

To illustrate this, we consider the four axiotaxy components that are observed for NiSi on $\mathrm{Si}(001)$. The two most

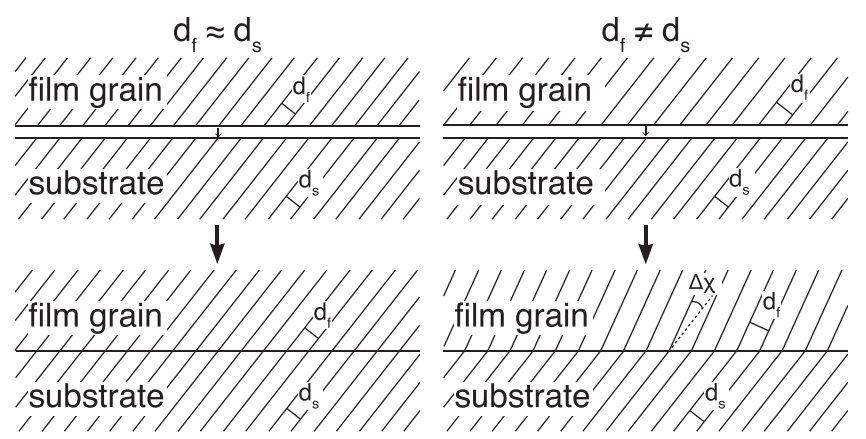

FIG. 10. Illustration of how a low-index film plane and a low-index substrate plane can be matched at the interface to create a one-dimensional periodic interface structure. Such a preferential grain orientation is called axiotaxy. If the matching planes have identical d-spacings, then they will be aligned across the interface (situation on the left). For planes with different lattice spacings, a relative tilt can still induce matching at the interface, but the planes will not be aligned (situation on the right). In practice, axiotaxy only seems to occur for aligned or nearly aligned planes (tilt angle $\Delta \chi<5^{\circ}$ ). 
intense components are caused by plane alignment across the interface between $\mathrm{NiSi}(211)$ or $\mathrm{NiSi}(202)$ and $\mathrm{Si}(220)$, characterized by a difference in d-spacing between film and substrate planes of merely $0.05 \%$ for both NiSi planes. The two other components are only weakly visible in pole figures and are caused by a matching at the interface between $\mathrm{NiSi}(103)$ or $\mathrm{NiSi}(112)$ and $\mathrm{Si}(220)$ planes. The difference in d-spacing (at room temperature) between these $\mathrm{NiSi}$ planes and $\mathrm{Si}(220)$ is -7.6 and $+3 \%$ for $\mathrm{NiSi}(103)$ and $\mathrm{NiSi}(112)$, respectively. For these orientations, the center of the axiotaxy circles has been observed at $\chi=40.8^{\circ}$ and $\chi=46.5^{\circ}$. As the $\operatorname{Si}(220)$ pole is located at $\chi=45^{\circ}$, this means that the $\mathrm{NiSi}(103)$ and $\mathrm{NiSi}(112)$ planes have a tilt $\Delta \chi$ of -4.2 and $+1.5^{\circ}$, respectively, reducing the difference in the projected d-spacing with $\operatorname{Si}(220)$ to less than $0.01 \%$ in both cases, ${ }^{31,51}$ which creates the one-dimensional interface match as sketched in the left column of Fig. 10. The fact that these two last components are only weakly observed illustrates the general observation that axiotaxy preferably occurs when the interface match can be achieved through plane alignment $\left(d_{f} \approx d_{s}, \Delta \chi \approx 0\right)$ and is less likely to be observed when larger tilt angles are needed to obtain the match at the interface. In practice, axiotaxy components where tilt angles larger than $5^{\circ}$ are needed to ensure interface matching have not been observed.

To understand why axiotaxy is only observed when the matching between lattice planes of film and substrate is achieved through plane alignment or through very small tilt angles, Detavernier et al. considered the robustness of the formed one-dimensional periodic interface structure with respect to interfacial roughness. ${ }^{31}$ The insets of Fig. 11 clearly show that for perfect plane alignment across the interface, the 1D periodic match is ensured irrespective of the interface curvature, while for a one-dimensional match obtained through a tilt angle $\Delta \chi \neq 0$ the match at the

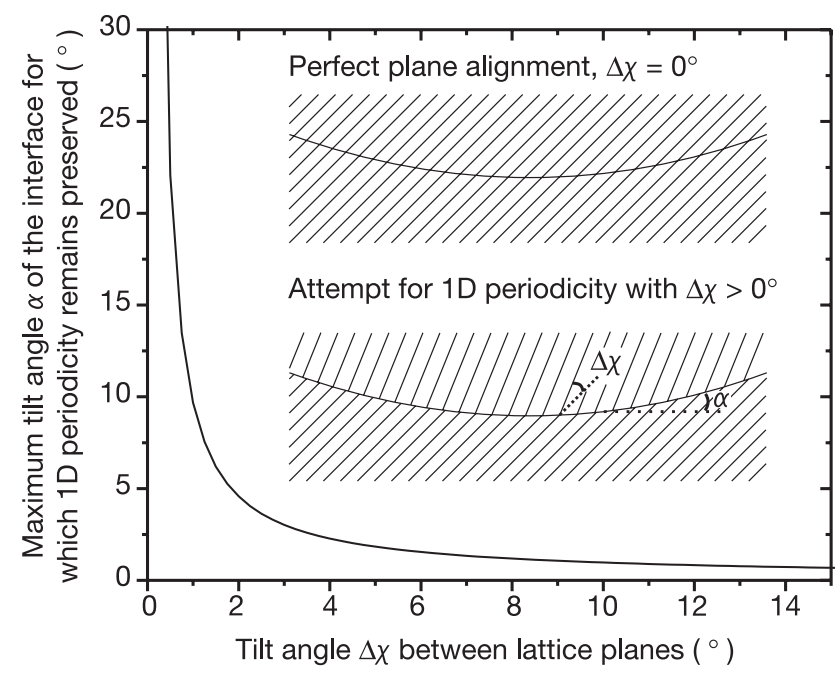

FIG. 11. Maximum tilt angle $\alpha$ through which the interface can curve while maintaining good periodicity (defined as a maximum mismatch of $0.5 \%$ between the projected d-spacing of film and substrate planes) as a function of the tilt angle $\Delta \chi$ of the film planes. The larger the allowed value of $\alpha$, the more stable the one-dimensional periodic interface structure is with respect to interfacial curvature. Reprinted with permission from Detavernier et al., Nature 426, 641 (2003). Copyright 2003 Nature Publishing Group. interface deteriorates as the interface tilt angle $\alpha$ increases. The graph in Fig. 11 shows the maximum interface tilt that still preserves a good periodic interface match (defined as a maximum difference of $0.5 \%$ in projected d-spacing) as a function of the tilt angle $\Delta \chi$ between the film and substrate planes. This clearly shows that for increasing $\Delta \chi$, the range of curvature over which the interface maintains good periodicity shrinks dramatically. The fact that axiotaxy is only observed for small tilt angles $\Delta \chi$ is probably related to the irregularly shaped interface that is present during the nucleation of a new phase during a solid-state reaction between the film and the $\mathrm{Si} / \mathrm{Ge}$ substrate. This will be discussed in more detail in Sec. III C 2.

Due to the one-dimensional match within the interface plane for an axiotaxy texture, the different grains belonging to an axiotaxy texture component are distributed with varying rotations around the axiotaxy axis, which results in the observed circular patterns when measuring pole figures. However, when glancing over the different axiotaxy containing pole figures included in this review or in the references included in Table I, one can observe that the intensity along such an axiotaxy circle is rarely uniform. This can be understood by considering that for grains belonging to the same axiotaxy component, a different film plane will be parallel to the interface depending on the rotation around the axiotaxy axis. For any such rotation, the interface will at least be periodic in one direction (due to the axiotaxy-related plane alignment), but some rotations around the axiotaxy axis might result in periodicity along a second, independent direction within the plane of the interface, resulting in an even lower interface energy. As a result, a larger fraction of the axiotaxy grains will orient in this way, resulting in a higher intensity on the axiotaxy rings for those rotations around the axiotaxy axis. If the periodicity in the second direction is welldefined, a two-dimensional periodic interface structure is obtained, and the subset of grains having this orientation will cause well-defined epitaxy spots on the pole figures at certain positions along the axiotaxy lines. As such, these grains constitute an epitaxial texture component that is a subset of the underlying axiotaxy texture. In some cases, such epitaxial spots are observed at the crosspoint between axiotaxy circles from two different axiotaxy components. In this case, the two determining constraints for the epitaxial alignment are defined by the two axiotaxy relations and the epitaxy is referred to as double axiotaxy (see, e.g., Section III D 2 on $\mathrm{CoSi}_{2}$ texture). From the arguments above, one can expect an epitaxial texture component that is a double axiotaxy to be unaffected by interface roughening (e.g., in the case of agglomeration), whereas an epitaxy that results from mere interface matching will disappear in case of interface roughening.

\section{Texture selection during solid state reactions}

So far, axiotaxy has mainly been observed in materials that are formed through solid-state reactions between a thin film and an underlying substrate. For silicides, observations are reported for $\mathrm{NiSi}$ on $\mathrm{Si}(001), \mathrm{Si}(110)$ and $\mathrm{Si}(111),{ }^{51}$ $\mathrm{CoSi}_{2}$ on $\mathrm{Si}(001), \mathrm{Si}(110)$ and $\mathrm{Si}(111),{ }^{67} \alpha-\mathrm{FeSi}_{2}$ on $\mathrm{Si}(001)$, 
and $\mathrm{CrSi}_{2}$ on $\mathrm{Si}(001) .{ }^{77}$ In the case of germanides, axiotaxy has been reported for $\mathrm{NiGe}$ on $\mathrm{Ge}(001)$ and $\mathrm{Ge}(111),{ }^{83}$ $\mathrm{Co}_{5} \mathrm{Ge}_{7}$ and $\mathrm{CoGe}_{2}$ on $\mathrm{Ge}(001)$ and $\mathrm{Ge}(111),{ }^{42}$ and PdGe on $\mathrm{Ge}(001)$ and $\mathrm{Ge}(111) .{ }^{87}$ Beside the typical observations in silicide/germanide materials, axiotaxy has also been observed more recently in $\mathrm{Ti}_{2} \mathrm{AlC}$ films formed on (0001), (1010), and (1102) oriented sapphire substrates ${ }^{104}$ and for $\mathrm{MnP}$ nanoclusters formed in $\mathrm{GaP}$ epilayers on $\mathrm{GaP}(001)$ substrates. ${ }^{105}$ All these results have in common that the materials under consideration are formed through solid-state reactions that are governed by (long range) solid-state diffusion and nucleation. Regarding texture selection during these types of reactions, there are two important points to consider.

First, the formation of a new phase in the phase sequence during a solid-state reaction starts with the nucleation of very small nuclei of the new phase at the interface between the preceding phase and the substrate. ${ }^{106}$ Even if the barrier for nucleation is low and diffusion becomes the rate-limiting factor, the nucleation must still occur. This means that the orientation of the new grains and thus the texture of the film will be determined at the interface between the nuclei of the new phase and the substrate. According to the classical theory of nucleation, the change in free energy associated with the creation of a spherical nucleus of a new phase (e.g., a metal silicide MSi) with radius $r$ at the interface between the metal film $\mathrm{M}$ and the $\mathrm{Si}$ substrate is determined by a competition between two energy contributions. On the one hand, there is a "gain" in volume free energy $\Delta G_{V} \sim r^{3} \Delta G$ as the formation of the phase MSi is enabled by its lower volume free energy $\Delta G$ compared to the separate phases $\mathrm{M}+\mathrm{Si}$. On the other hand, there is a surface energy "cost" $\Delta \sigma_{A} \sim r^{2} \Delta \sigma$ associated with the change in interface energy $\Delta \sigma$ due to the creation of the MSi nucleus. The total free energy change for a nucleus with radius $r$ is thus given by

$$
\Delta G_{N}(r)=a r^{2} \Delta \sigma-b r^{3} \Delta G
$$

Here, $a$ and $b$ are constants that account for the reality of a nucleus being not perfectly spherical. This evolution of the free energy $\Delta G_{N}$ with the nucleus radius $r$ is illustrated in Fig. 12, where the surface energy "cost" and the volume energy "gain" are shown together with the combined free energy change $\Delta G_{N}$. From Equation (1) and Fig. 12, it is clear that there is an energy barrier $\Delta G^{*}$ for nucleation to proceed with a corresponding critical nucleus radius $r^{*}$ given by

$$
\begin{aligned}
r^{*} & \sim \frac{\Delta \sigma}{\Delta G}, \\
\Delta G^{*} \sim \frac{\Delta \sigma^{3}}{\Delta G^{2}} & \sim \frac{\Delta \sigma^{3}}{(\Delta H-T \Delta S)^{3}} .
\end{aligned}
$$

From the arguments above, one can see that the nucleation of a new phase can be facilitated by reducing the energy "cost" $\Delta \sigma_{A}$, which can be achieved in a couple of ways. First, nucleation of grains of a new phase typically

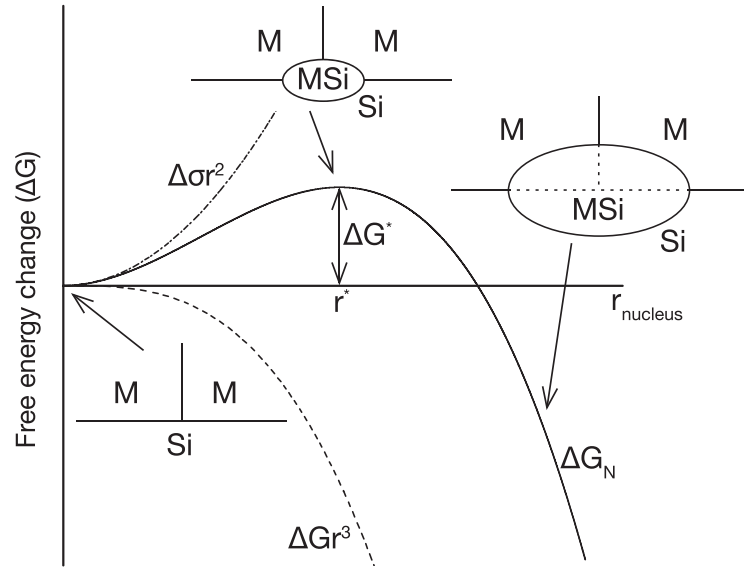

FIG. 12. Evolution of the free energy change associated with the creation of a nucleus with radius $r$. The contributions of the surface energy "cost" $\Delta \sigma_{A}$ and the volume energy gain $\Delta G_{V}$ are drawn separately. The competition between these opposing effects gives rise to an energy barrier $\Delta G^{*}$ (and an associated critical radius $r^{*}$ ) that a growing nucleus must overcome before it can continue to grow.

takes place at the grain boundaries (see sketches in Fig. 12) as this results in the removal of the grain boundary interface energy. Second, the orientation of the newly formed nucleus with respect to the substrate directly impacts the interface energy. This is where texture comes into play, e.g., an epitaxial grain orientation will result in a very low interface energy due to the two-dimensional periodic interface structure. Last, if the low interface energy of an epitaxial orientation is not accessible, a forming nucleus can still reduce the energy cost $\Delta \sigma_{A}$ by obtaining a curved interface as this minimizes the interface area between the new phase and the surrounding phase(s) and substrate. In this case, an axiotaxial grain orientation can form at this curved interface as was discussed above, resulting in an extra reduction in interface energy.

Second, Equation (3) shows that the activation energy for the nucleation of a new silicide/germanide phase at the interface of the preceding phase with the $\mathrm{Si} / \mathrm{Ge}$ substrate varies as $\Delta \sigma^{3}$ and $1 / \Delta G^{2}$, with $\Delta G=\Delta H-T \Delta S$ the free energy change for the reaction between the substrate and the preceding phase into the new phase. In a typical binary solid-state reaction, the entropy change $\Delta S$ is small compared to $\Delta G$, and thus, the enthalpy change $\Delta H$ can be taken as a good measure for $\Delta G$. From this $\Delta \sigma^{3} / \Delta H^{2}$ dependence of the activation energy for nucleation, it follows that for phases having a low enthalpy change, the contribution of the interface energy becomes much more important. ${ }^{106}$ As a result, for these phases, the preferential grain orientation has a high impact on the nucleation barrier. This explains the observation that, when considering literature reports on texture in thin silicide/germanide films over the past decade, texture is typically much more pronounced for phases that appear at a later stage in a phase formation sequence, as they are usually characterized by a smaller enthalpy change $(\Delta H)$ compared to the phase(s) that appear(s) first. ${ }^{106}$

Up until this point, the focus of this discussion has been on nucleation. However, one might argue that diffusion processes are equally important in the kind of solid-state 
reactions under discussion when the activation energies for both kinds of processes are comparable. For example, the identity of the dominant diffusing species is considered an important parameter in silicidation and germanidation reactions. When focussing on the texture evolution during such a reaction, one might intuitively argue that axiotaxy is more likely when the metal is the diffusing species. While this is true, e.g., for the formation of $\mathrm{NiSi}$ or $\mathrm{CoSi}_{2}$ where the metal is the dominant diffusing species and an axiotaxial grain orientation is indeed observed, the opposite is observed in the Ti-Si system where $\mathrm{Si}$ is the dominant diffusion species during the formation of $\mathrm{TiSi}_{2}$ and an axiotaxial alignment is also obtained (see par. III D 1). In general, the influence of diffusion processes on the texture evolution during these solidstate reactions is currently far from understood and further investigation is needed. For nucleation however, the connection with texture evolution is much clearer (as was discussed above): it is at nucleation that a grain will fix its orientation. Even when the barrier to nucleation is low, it must still occur. So far, there has only been one, very recent observation reported in literature of a silicidation reaction where the orientation of the grains of a single phase changes after nucleation. For $50 \mathrm{~nm}$ Pd films deposited on $\mathrm{Si}(001)$, Richard et al. ${ }^{107}$ observed the formation of a peculiar texture when the $\mathrm{Pd}_{2} \mathrm{Si}$ phase forms. The initially formed $\mathrm{Pd}_{2} \mathrm{Si}$ layer showed four orientation variants and a large tilt $\left(19.5^{\circ}\right)$ of the $c$-axis with respect to the surface normal. After the formation of a continuous $\mathrm{Pd}_{2} \mathrm{Si}$ film, the grains were found to collectively rotate during a further heat treatment. This rotation was argued to result from the drive to lower the grainboundary (GB) energy of the high-energy GBs that result from the initial grain orientation variants. This lowering of GB energy is believed to occur through GB diffusion. As such, this study and more specifically the introduction of in situ pole figure acquisition during heat treatments might be the starting point for future investigations concerning the link between texture evolution and diffusion processes during silicidation and germanidation reactions.

\section{Texture of $\mathrm{TiSi}_{2}, \mathrm{CoSi}_{2}$, and $\mathrm{NiSi}$}

In order for a silicide or germanide to be useful as a contacting material in MOSFET devices, the material has to fulfill a number of requirements such as low electrical resistivity, low contact resistance, good thermal stability, etc. (see Ref. 108 for a full list of requirements). Even if the silicide's bulk properties are acceptable, often it has proved to be challenging to form the required phase in the small contact areas where they are needed. Over the years, three silicides have found extensive use as contacting materials in MOSFET devices, i.e., C54-TiSi $2, \mathrm{CoSi}_{2}$, and NiSi. Below, we will briefly describe the texture of thin films of these materials when formed through a solid state reaction between a thin metal layer ( $\mathrm{Ti}, \mathrm{Co}$, or $\mathrm{Ni}$ ) and a single crystalline silicon substrate.

\section{1. $\mathrm{TiSi}_{2}$}

The first silicide to be used on a large scale with the SALICIDE process was $\mathrm{TiSi}_{2}$. There are two polymorphs of this phase, i.e., base-centered orthorhombic $\mathrm{C} 49-\mathrm{TiSi}_{2}$ (JCPDS 23-964, $\quad a=0.3562 \mathrm{~nm}, \quad b=1.353 \mathrm{~nm}, \quad$ and $c=0.355 \mathrm{~nm})$ and face-centered orthorhombic $\mathrm{C} 54-\mathrm{TiSi}_{2}$ (JCPDS 35-0758, $a=0.8268 \mathrm{~nm}, \quad b=0.8553 \mathrm{~nm}$, and $c=0.4798 \mathrm{~nm}$ ). Of these two, the C54 phase has by far the lowest resistivity and thus is the polymorph needed in the contact regions. For devices with feature sizes larger than $250 \mathrm{~nm}$, different techniques were developed to form this low-resistive C54 phase. ${ }^{109}$ For smaller devices, the nucleation controlled transformation from $\mathrm{C} 49$ (which forms before C54 in the solid state reaction) to C54 is inhibited, making it very difficult to form the desired $\mathrm{C} 54-\mathrm{TiSi}_{2}$. Thus, for devices with feature sizes below $250 \mathrm{~nm}$, a process using an alternative silicide such as $\mathrm{CoSi}_{2}$ or $\mathrm{NiSi}$ had to be developed.

Early studies on texture of $\mathrm{C} 54-\mathrm{TiSi}_{2}$ thin films on silicon date back to the late $1980 \mathrm{~s} .{ }^{55-58}$ These studies focussed mainly on the texture of $\mathrm{C} 54$ on $\mathrm{Si}(111)$ as it was observed that the growth of this phase on $\mathrm{Si}(111)$ was mainly epitaxial and the interest in epitaxial silicides was high at that time. These studies mainly used XRD $(\theta / 2 \theta$ and grazing incidence) and electron diffraction techniques to identify two main epitaxial texture components for C54 on $\mathrm{Si}(111)$ : (1) C54(110)// $\mathrm{Si}(111)$ and $\mathrm{C} 54(\overline{3} 31) / / \mathrm{Si}(2 \overline{2} 0)$ and (2) $\mathrm{C} 54(100) / / \mathrm{Si}(111)$ and $\mathrm{C} 54(040) / / \mathrm{Si}(02 \overline{2})$.

Epitaxial growth of $\mathrm{C} 54$ on $\mathrm{Si}(001)$ was also studied by several groups during the 1990s. ${ }^{59-62}$ It was found that C54 epitaxy on $\mathrm{Si}(001)$ is more difficult than on $\mathrm{Si}(111)$, especially for $\mathrm{C} 54$ films grown by a solid-state reaction from $\mathrm{Ti}$ films deposited at low temperatures. In 2002, Özcan et al. performed a detailed study on the texture of the C54 (and C49) phase formed on $\mathrm{Si}(001)$ by using synchrotron based $\mathrm{X}$-ray diffraction (see Fig. 13). ${ }^{30}$ For C54 films grown on $\mathrm{Si}(001)$ from both sputter- and CVD-deposited Ti films, they observed epitaxial components in which a low-index C54 plane is parallel to a $\mathrm{Si}\{111\}$ plane (similar to the observed epitaxies of $\mathrm{C} 54$ on $\mathrm{Si}(111)$ ) instead of parallel to the $\mathrm{Si}(100)$ interface plane. They explained these observed orientations by ledging of the substrate on $\{111\}$ planes during silicide formation. Next to these epitaxial texture components, more recent, yet unpublished high-resolution pole figure data collected by our respective groups also show the presence of axiotaxy in C54-TiSi ${ }_{2}$ films on $\mathrm{Si}(100)$.
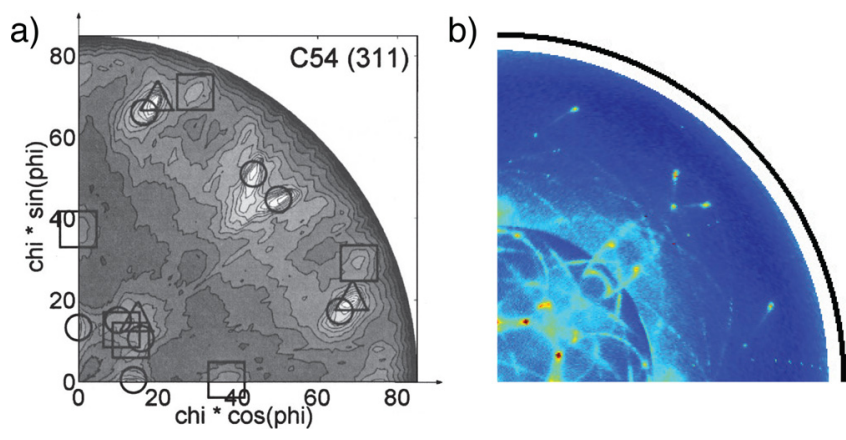

FIG. 13. Selection of $\mathrm{TiSi}_{2}$ pole figures with a) $\mathrm{TiSi}_{2}(311)$ on $\mathrm{Si}(100)$ (reprinted with permission from J. Appl. Phys. 92, 5011 (2002). Copyright 2002 AIP Publishing LLC.) and (b) $\mathrm{TiSi}_{2}(311)$ on $\mathrm{Si}(100)$ measured with high resolution pole figure setup. 


\section{2. $\mathrm{CoSi}_{2}$}

When feature sizes in MOSFET devices dropped below $250 \mathrm{~nm}, \mathrm{CoSi}_{2}$ became the contacting silicide of choice as it does not suffer from the nucleation problems observed with $\mathrm{TiSi}_{2}$ contacts in these smaller lines. ${ }^{17}$ This silicide was used until linewidths in devices reached $50 \mathrm{~nm}$ and several issues with $\mathrm{CoSi}_{2}$ formation in these narrow lines arose: ${ }^{18}$ void formation, the inability to form $\mathrm{CoSi}_{2-x} \mathrm{Ge}_{x}$ phases, and the higher formation temperature of $\mathrm{CoSi}_{2}$ when $\mathrm{Ge}$ is introduced in the source and drain regions and the high silicon consumption for $\mathrm{CoSi}_{2}$ formation, which can be problematic when using SOI (Silicon On Insulator) substrates.

Because of the small lattice mismatch $(\approx 1.2 \%)$ and similar crystal structure between $\mathrm{CoSi}_{2}$ (cubic $\mathrm{CaF}_{2}$ structure, $\mathrm{a}=0.536 \mathrm{~nm}$ ) and $\mathrm{Si}$ (cubic diamond structure, $\mathrm{a}=0.543 \mathrm{~nm}$ ), $\mathrm{CoSi}_{2}$ is expected to easily form epitaxially on $\mathrm{Si}$ substrates. As a result, a lot of effort was put in developing and optimizing methods to grow epitaxial $\mathrm{CoSi}_{2}$ layers during the 1980s and 1990s (MBE, OME/TIME, mesotaxy, see Section III A). However, it has proven surprisingly difficult to grow epitaxial layers of $\mathrm{CoSi}_{2}$ through a simple solid-state reaction between a thin Co film and a $\mathrm{Si}$ substrate, especially on $\mathrm{Si}(001)$. Such a reaction results in a polycrystalline $\mathrm{CoSi}_{2}$ film, the texture of which was first studied in detail by Bulle-Lieuwma et al. ${ }^{29}$ in 1992 using pole figures and TEM. They observed that forming $\mathrm{CoSi}_{2}$ on $\mathrm{Si}(001)$ through such a solid-state reaction resulted in different epitaxial components occurring simultaneously, which explained the polycrystalline nature of the films.

In 2004, Özcan et al. used high-resolution synchrotron based X-ray pole figure measurements to re-assess the texture of $\mathrm{CoSi}_{2}$ on $\mathrm{Si}(001) .{ }^{66}$ In addition to the epitaxial components, their detailed measurements allowed them to identify three axiotaxy components that result from the alignment of a $\mathrm{CoSi}_{2}\{110\}$-type of plane with a $\mathrm{Si}\{110\}$ type of plane. A few years later, De Keyser et al. used the same type of pole figure measurements to perform a detailed study of the texture of $\mathrm{CoSi}_{2}$ films on three substrate orientations, i.e., $\mathrm{Si}(001), \mathrm{Si}(110)$, and $\mathrm{Si}(111){ }^{67}$ Their results revealed a complex texture on all three substrate orientations, as evidenced by the complex patterns of epitaxial spots and axiotaxy lines visible on the $\mathrm{CoSi}_{2}(111)$ and (110) pole figures on different substrate orientations shown in Fig. 14. The axiotaxial alignment that was previously observed by Özcan et al. on $\mathrm{Si}(001)$ substrates ${ }^{66}$ was observed here on all three substrates. Furthermore, De Keyser et al. found that almost all observed epitaxial components were strongly related to the observed axiotaxy, with either one or both constraints of each epitaxial component arising from the axiotaxial alignment of the $\mathrm{CoSi}_{2}\{110\}$ and $\mathrm{Si}\{110\}$ planes across the interface. The case where both epitaxial constraints are linked to axiotaxy or plane alignment was termed "double axiotaxy" (see also Section III C). For a full discussion of all texture components observed in $\mathrm{CoSi}_{2}$ films, the reader is referred to Ref. 67.

\section{NiSi}

For technology nodes starting from $65 \mathrm{~nm}, \mathrm{NiSi}$ became the industry standard contacting material due to its low
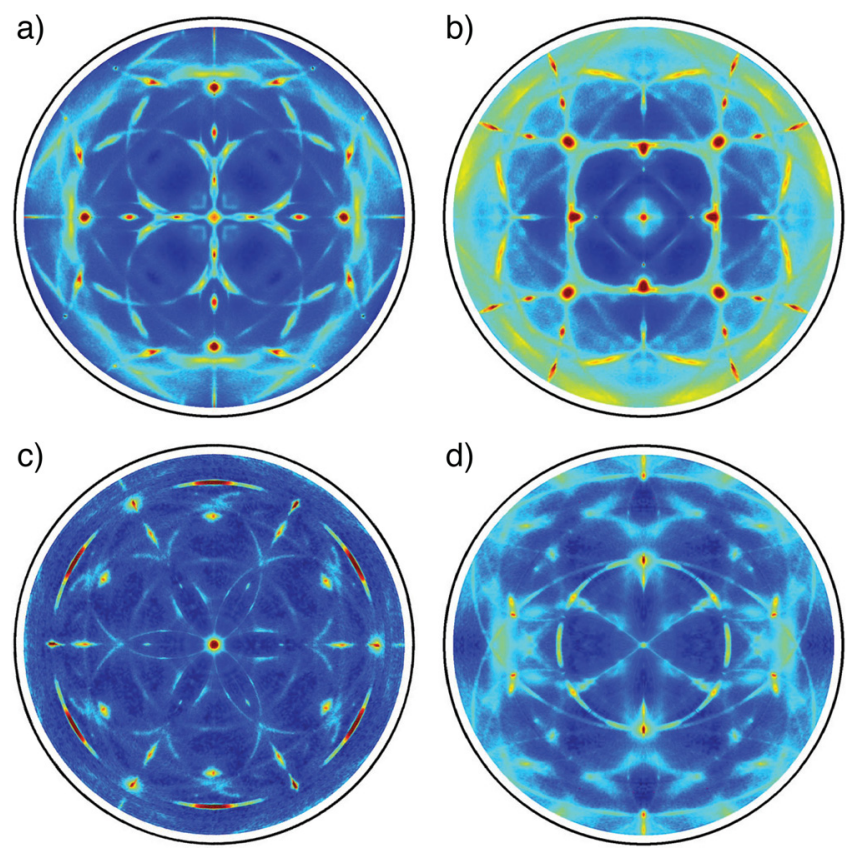

FIG. 14. Selection of $\mathrm{CoSi}_{2}$ pole figures with (a) $\mathrm{CoSi}_{2}(111)$ on $\mathrm{Si}(100)$, (b) $\mathrm{CoSi}_{2}(110)$ on $\mathrm{Si}(100)$, (c) $\mathrm{CoSi}_{2}$ (111) on $\mathrm{Si}(111)$, and (d) $\mathrm{CoSi}_{2}(111)$ on $\operatorname{Si}(110)$.

formation temperature, low resistivity, compatibility with $\mathrm{SiGe}$, low Si consumption (compared to $\mathrm{CoSi}_{2}$ and $\mathrm{TiSi}_{2}$ ), and absence of fine-line effects. However, there are two important issues with $\mathrm{NiSi}$ thin films, i.e., thermal stability (NiSi transforms into $\mathrm{NiSi}_{2}$ at higher temperatures) and morphological stability (NiSi films agglomerate at higher temperatures), the latter degradation mechanism being the most important for thin films $(<30 \mathrm{~nm}) .{ }^{110}$ In 1999, Mangelinck et al. ${ }^{19}$ discovered that alloying a NiSi film with Pt $(10-15$ at. \%) can improve its thermal stability substantially. In Section IV C, we will discuss how the texture of a NiSi film partly explains this stabilization through Pt alloying.

As NiSi was not one of the silicides considered for epitaxial thin film growth (unlike $\mathrm{NiSi}_{2}$, which has a very close match to $\mathrm{Si}$ ), little attention was given to its texture until it was considered as a possible replacement for $\mathrm{CoSi}_{2}$ at the $65 \mathrm{~nm}$ technology node. Because early studies showed that polycrystalline silicide films could display unusual and complex texture, ${ }^{29,30}$ interest in the texture of NiSi films increased dramatically. High resolution X-ray pole figure measurements performed at the IBM X20A beamline (Brookhaven National Lab, NY, USA) on thin NiSi films grown on $\mathrm{Si}(001)$ substrates were carried out and eventually led to the discovery of axiotaxy in $2003 .{ }^{31}$ In 2008 , Detavernier et al. published a detailed follow-up study on the texture of thin NiSi films on $\mathrm{Si}(001), \mathrm{Si}(110)$, and $\mathrm{Si}(111)$ substrates. Pole figures of NiSi films on these three substrates reveal a complex texture (see Fig. 15) that is a combination of axiotaxy and epitaxy components. On all three substrates, the bright patterns of lines are caused by two axiotaxy components for which either the $\mathrm{NiSi}\{202\}$ or $\{211\}$ planes are aligned with $\mathrm{Si}\{110\}$ planes across the interface. Two weaker axiotaxy components were also identified on $\mathrm{Si}(001)$ and $\mathrm{Si}(111)$. These components result from a match at the interface between either the 

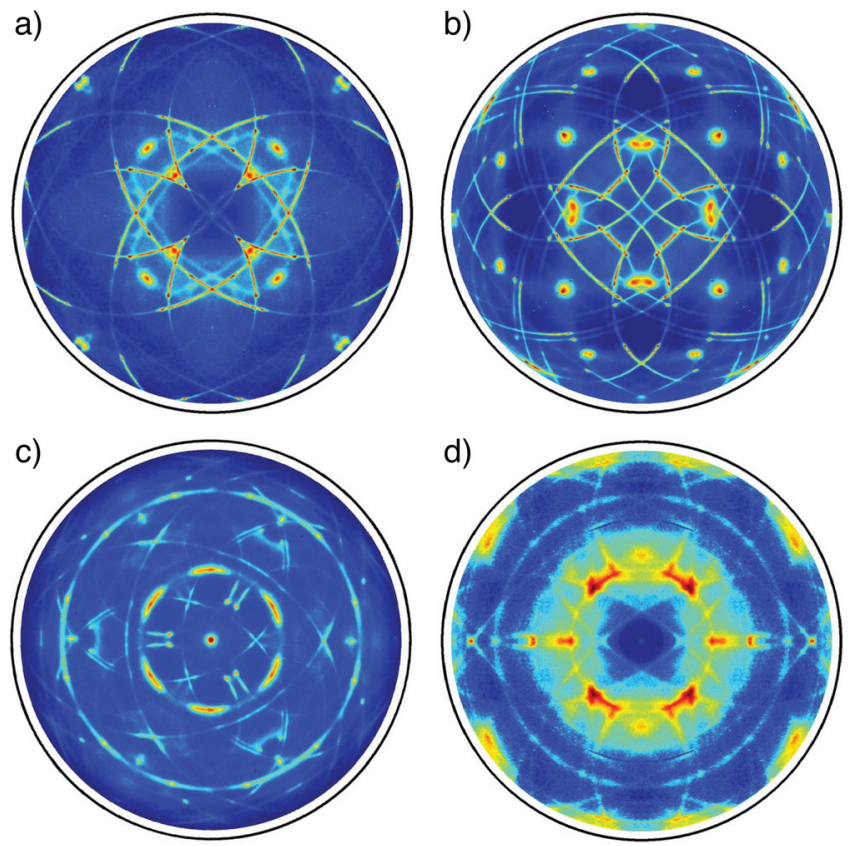

FIG. 15. Selection of NiSi pole figures with (a) NiSi(002)/(110) on $\mathrm{Si}(100)$, (b) $\mathrm{NiSi}(112)$ on $\mathrm{Si}(100)$, (c) $\mathrm{NiSi}(002) /(110)$ on $\mathrm{Si}(111)$, and (d) $\mathrm{NiSi}(002) /$ (110) on $\operatorname{Si}(110)$.

$\operatorname{NiSi}\{103\}$ or $\{112\}$ plane and the $\operatorname{Si}\{220\}$ plane which is made possible by a slight tilt of the off-normal fiber axis ${ }^{31,51}$ (resulting in a difference in projected d-spacing of less then $0.5 \%)$. Next to the axiotaxy, several epitaxial components were also identified on all three substrate types. Similar to the case of $\mathrm{CoSi}_{2}$ discussed above, some of these epitaxial components could be linked to the axiotaxy, i.e., one of the two epitaxial constraints is defined by the alignment of $\mathrm{NiSi}(211)$ or (202) and $\mathrm{Si}(220)$. However, for NiSi, no epitaxial components that result from "double axiotaxy" were observed. For a complete overview and discussion of the texture components observed in NiSi thin films, the reader is referred to Ref. 51.

In addition to the axiotaxy and epitaxy, a different microstructure has been reported to occur in thin NiSi films on $\mathrm{Si}(001)$. Alberti et al. observed the formation of so-called "transrotational" domains, both in $\mathrm{Ni}_{2} \mathrm{Si}$ and NiSi films. ${ }^{49,111}$ These domains result from a bending (either spherical or cylindrical) of specific crystallographic planes of the film in order to adapt to the crystal structure of the substrate. ${ }^{49}$ This transrotational NiSi has been observed to form under different experimental conditions (annealing ambient, substrate doping, etc.) as long as specific care is taken during the deposition of the initial $\mathrm{Ni}$ film: the Ni must be deposited at slightly elevated temperatures in order to obtain a Ni-rich intermixed Ni:Si layer and when the thickness of the deposited $\mathrm{Ni}$ surpasses $\sim 7 \mathrm{~nm}$, a low temperature isothermal anneal is necessary to form transrotational NiSi. Recently, Alberti et al. extensively reviewed the formation and properties of these transrotational Ni-silicides. ${ }^{112}$

\section{INFLUENCE OF TEXTURE ON SILICIDE/ GERMANIDE FORMATION AND PROPERTIES}

As was mentioned in Secs. I and III, contact formation in CMOS fabrication happens through a solid-state reaction between a thin metal film and the $\mathrm{Si}$ or Ge substrate. The formation of a thin silicide/germanide film through such a solid-state reaction proceeds through a complex interplay of silicon and/or metal diffusion and nucleation of new silicide phases. Texture can greatly influence this process as the orientation of the individual grains determines the type and orientation of grain boundaries (affecting diffusion) and the interface energy (affecting nucleation). For similar reasons, texture will also influence the stability of these films against either agglomeration (breaking-up of the film into islands, destroying the low resistance) or transformation into a thermodynamically more stable silicide (e.g., transformation of $\mathrm{NiSi}$ to $\mathrm{NiSi}_{2}$ at high temperatures).

A high quality silicide/germanide contact is characterized by functional properties such as low sheet resistance, low contact resistance, low stress, appropriate Schottky barrier height, etc. ${ }^{7}$ With the continued reduction in feature sizes, the contact formation scheme has evolved from a self aligned silicide contact to what is referred to as a trench contact. Here, the silicide is formed at the bottom of a very narrow trench $(<20 \mathrm{~nm}$ in width) and then filled with a conductive metal. As a result, the resistivity of the silicide itself is much less critical since the current can be carried laterally in the metal. However, because of the size reduction, the intrinsic contact resistance of the silicide/Si interface becomes the dominant factor in the external resistance of devices. Thus, understanding and controlling interface properties becomes crucial. As such, properties like Schottky barrier height, doping concentrations, interfacial bonding, and effects of impurities must be controlled in dimensions that become smaller than a typical grain.

Over the years, film texture has been shown to affect all of these interfacial properties. As the bonding with the Si/Ge substrate varies with film orientation, interfacial properties will vary with film texture. Furthermore, many of these properties will be affected by the anisotropy of the film lattice. The manifestation of this anisotropy on the macroscopic level will depend on how the individual grains in the film are oriented, i.e., on the texture. One can imagine that for films consisting mainly of epitaxially aligned grains, the anisotropy can be maintained macroscopically. For a randomly textured film or a film with many different texture components, the anisotropy will be averaged out, and the film will display isotropic behavior on the macroscopic scale with possible local variations due to the different components. As an example, the NiSi lattice is extremely anisotropic and shows very large thermal expansion coefficients with even larger variations depending on the crystal axis (from about $40 \mathrm{ppm} /{ }^{\circ} \mathrm{C}$ to about $\left.-40 \mathrm{ppm} /{ }^{\circ} \mathrm{C}\right) .{ }^{113}$ This will cause local variations in strain at the interface.

Clearly, the influence of texture on the formation, the stability, and general properties of these thin silicide/germanide films can be significant. Texture studies using pole figures or EBSD can provide conclusive information when identifying silicide/germanide phases formed during a solidstate reaction. This is especially the case when the formed phase exhibits strong epitaxial or fiber components, as standard $\theta / 2 \theta$ XRD techniques provide limited information for such films ${ }^{37}$ (see also Section II A). In this section, we will 
give an overview of studies that have investigated the influence of texture on silicide/germanide phase formation and properties.

\section{A. Influence on phase formation}

In literature on thin film silicides and germanides, the concept of "phase formation" refers to the sequence of silicide/germanide phases that form during the solid-state reaction between the metal film and the silicon/germanium substrate. An efficient technique to identify the different silicide/germanide phases that form during the reaction is in situ $\mathrm{X}$-ray diffraction, where a linear detector is used to repeatedly capture an X-ray diffraction spectrum with a set timeinterval while the sample is heated to a specific temperature at a fixed heating rate. However, as was mentioned earlier, the fixed $\theta / 2 \theta$ geometry used in this measurement only represents a very small subset of possible diffraction positions and limits our ability to detect phases that are highly textured. In a case where ambiguous diffraction peaks or no diffraction peaks are detected at a given temperature, pole figure or EBSD measurements performed on samples quenched at that specific temperature help to unequivocally identify the crystal structure present at that stage of the reaction.

We will illustrate this point by using the phase formation sequence during the reaction of a thin $\mathrm{Ni}$ film on $\mathrm{Si}$. Because of the importance of $\mathrm{NiSi}$ as a contacting material in modern CMOS devices, this Ni-Si system has received a lot of attention in literature. Phase formation in this system has been extensively studied over the past three decades using TEM based techniques in the older studies (1980s and 1990s) and in situ XRD in the more recent studies (late 1990s and 2000s), often complemented with additional techniques like RBS (for elemental depth distributions), laser light scattering (for monitoring film roughness) and resistivity measurements. ${ }^{18,47,48,114-117}$ Most of these studies focussed on phase formation on a $\mathrm{Si}(001)$ substrate, as this is technologically the most relevant orientation. Fig. 16 shows an in situ XRD measurement for a $10 \mathrm{~nm}$ Ni film on $\mathrm{Si}(001)$ which clearly shows a complex phase formation sequence before the formation of the wanted $\mathrm{NiSi}$ phase. The

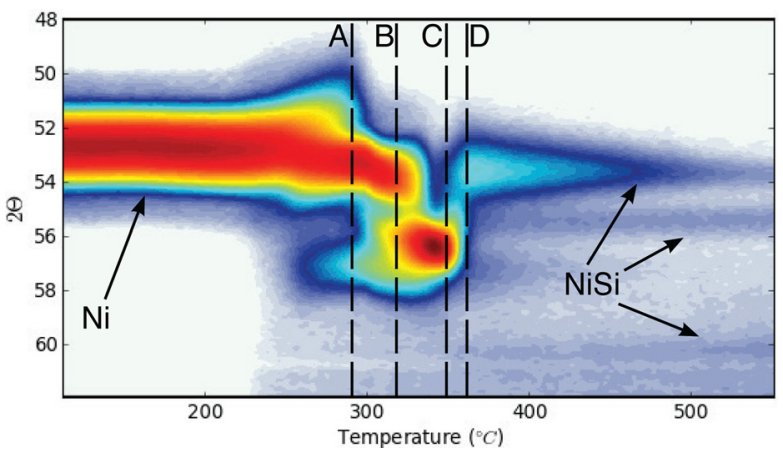

FIG. 16. In situ XRD $(\lambda=0.18 \mathrm{~nm})$ on a $10 \mathrm{~nm}$ Ni film on $\mathrm{Si}(001)$ measured at a heating rate of $1^{\circ} \mathrm{C} / \mathrm{s}$, showing the XRD peaks as a function of temperature. A complex phase formation sequence can be observed between the asdeposited Ni and the wanted NiSi phase. The dashed lines mark the temperatures where pole figures were measured by Gaudet et al. ${ }^{35}$ (see Fig. 17). relatively low amount of observed peaks and the overlap of different peaks made the identification of the phases not straightforward. By the mid 2000s, consensus was more or less reached on the co-existence of multiple metal-rich phases (observations of $\delta-\mathrm{Ni}_{2} \mathrm{Si}, \mathrm{Ni}_{31} \mathrm{Si}_{12}$, and $\mathrm{Ni}_{3} \mathrm{Si}_{2}$ were reported) that are possibly highly textured. ${ }^{117,118}$

However, the growing interest in the texture of these thin silicide films during the past decade and the corresponding development of high resolution XRD pole figure measurements on such films drove researchers to reassess the complex metal-rich phase formation sequence in the $\mathrm{Ni}$ Si system. In 2010, Gaudet et al. published a detailed study on this subject using in situ XRD combined with highresolution pole figures measured on samples quenched at different temperatures during the solid state reaction (see Figures 16 and 17). According to the observations of Gaudet et al., the as-deposited $\mathrm{Ni}$ displays a strong fiber texture with the $\mathrm{Ni}(111)$ plane being parallel to the substrate, which is evidenced by the spot in the center and the ring at $\chi=$ $70.5^{\circ}$ (= angle between $\mathrm{Ni}\{111\}$ planes) in the $\mathrm{Ni}(111)$ pole figure (top left in Fig. 17). Upon annealing, the reaction commences with the formation of orthorhombic $\delta-\mathrm{Ni}_{2} \mathrm{Si}$ (Pnma, $a=0.499 \mathrm{~nm}, \quad b=0.372 \mathrm{~nm}$, and $c=0.706 \mathrm{~nm}$ ), exhibiting two strong fiber components (see Fig. 17, quench at $\left.290^{\circ} \mathrm{C}\right)$ having either a $\{013\}(d=0.199 \mathrm{~nm})$ or a $\{020\}$ $(d=0.186 \mathrm{~nm})$ plane parallel to the substrate. Diffraction peaks of these planes are visible in the in situ XRD measurement in Fig. 16 at $53.6^{\circ}$ and $57.6^{\circ}$. In addition to the $\delta-\mathrm{Ni}_{2} \mathrm{Si}$ phase, the pole figure measurements performed by Gaudet et al. revealed a second phase with a strong epitaxial texture (marked as $\mathrm{Ni}_{x} \mathrm{Si}_{y}$ in Fig. 17) which is therefore bound to form at the interface with the single-crystal substrate. The precise nature of this phase is still an open question as no known phase from the $\mathrm{Ni}-\mathrm{Si}$ phase diagram is able to explain the observed epitaxial spots in the pole figures. ${ }^{33,35}$ Due to the specific epitaxial orientation of this unknown phase, it was never observed in earlier studies using standard in situ XRD.

After the formation of $\delta-\mathrm{Ni}_{2} \mathrm{Si}$ and the unknown epitaxial phase, the reaction continues with the formation of another phase, characterized by the strong diffraction peak around $56^{\circ}-57^{\circ}$ in the in situ XRD measurement of Fig. 16. In earlier studies, this peak was attributed to a strongly textured $\mathrm{Ni}_{3} \mathrm{Si}_{2}$ phase, the (350) plane of which could cause the diffraction peak observed in standard in situ XRD measurements. However, the pole figure measurements performed by Gaudet et al. revealed that this peak is caused by another phase with a strong fiber texture (see Figs. 17, B3, and C3). Detailed analysis of the observed features in the pole figures allowed to identify the $\theta$-nickel-silicide phase formed with a strong $\{110\}$ fiber texture. This $\theta$ phase is a non-stoichiometric compound with a hexagonal crystal structure (spacegroup $\left.P 6_{3} / m m c\right)$ in which a variable amount of $\mathrm{Ni}$ atoms can be present depending on the locations they occupy in the lattice, resulting theoretically in a possible $\mathrm{Si}$ content ranging between $33 \%$ and 55\%. However, according to the $\mathrm{Ni}-\mathrm{Si}$ binary phase diagram, this $\theta$ phase is only thermodynamically stable above $825^{\circ} \mathrm{C}$ when it contains between $33 \%$ and $41 \%$ 


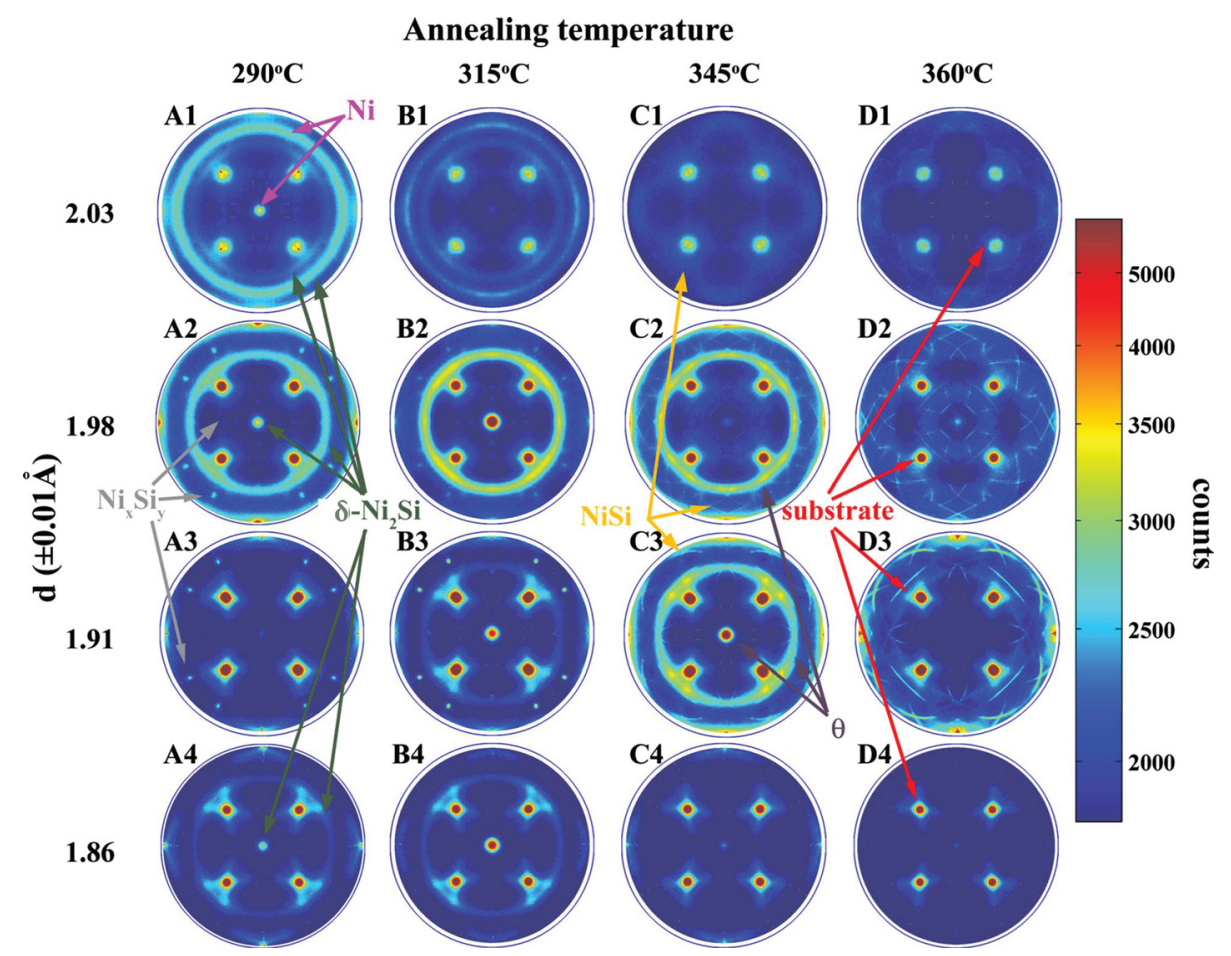

FIG. 17. XRD pole figures for four different $d$-spacings $(\lambda=0.15406 \mathrm{~nm})$ measured on samples quenched at different temperatures during the reaction of a $10 \mathrm{~nm}$ Ni film on $\mathrm{Si}(001)$. Figure reprinted with permission from $\mathrm{J}$. Appl. Phys. 107, 093515 (2010). Copyright 2010 AIP Publishing LLC.
$\mathrm{Si},{ }^{119}$ making the formation of this metastable phase at low temperatures rather unexpected.

Although the formation of this $\theta$ phase is thermodynamically unexpected at these low temperatures, its observation by Gaudet et al. during the solid-state reaction of a thin $\mathrm{Ni}$ film on $\mathrm{Si}(001)$ was actually consistent with the earlier results of De Keyser et al. in 2008, who observed the formation of this metastable phase when studying the solid-state reaction between co-sputtered $\mathrm{Ni}-\mathrm{Si}$ films with varying $\mathrm{Si}$ concentrations ( $\mathrm{Si}$ content between 37 and 42 at. \%, Ni content equivalent to a $50 \mathrm{~nm} \mathrm{Ni}$ film) and a $\mathrm{Si}(001)$ or (111) substrate. ${ }^{46}$ Because their in situ XRD patterns on both substrate orientations showed temperature windows with little or no diffraction peaks, they turned to pole figure and EBSD measurements and identified the presence of an epitaxially oriented $\theta$ phase which forms from the mixed Ni-Si film and reacts into $\mathrm{NiSi}$ at higher temperatures on both $\mathrm{Si}(001)$ and (111) substrates. These epitaxial orientations on both substrate types seemed to be closely related as they both have a $\theta\{0001\}$ basal plane parallel (or nearly parallel in the case of $\mathrm{Si}(001))$ to a $\mathrm{Si}\{111\}$ plane. ${ }^{46}$ The alignment of these planes can be understood by considering the small mismatch $(\approx 0.9 \%)$ in the distance between neighbouring $\mathrm{Si}$ atoms in the $\theta\{0001\}$ basal plane and the $\operatorname{Si}\{111\}$ plane. ${ }^{46}$ According to De Keyser et al., the nucleation of this metastable $\theta$ phase can be attributed to a combination of interface stabilization, thanks to the epitaxial orientation (low interface energy) and the fact that the $\theta$ phase can crystallize congruently with limited diffusion from the amorphous co-deposited Ni-Si mixture, as the $\theta$ phase can grow in exactly the same composition as this mixture. Furthermore, Gibson et al. and Gaudet et al. both reported the existence of very thin layers $(<2 \mathrm{~nm})$ of this $\theta$ phase at the interface with the same epitaxial orientation as reported by De Keyser et al. when depositing pure $\mathrm{Ni}$ on $\mathrm{Si}(111)$, which can probably be attributed to the thin amorphous $\mathrm{Ni}-\mathrm{Si}$ mixed layer that forms at the interface during $\mathrm{Ni}$ deposition. ${ }^{33,46,120}$

The influence of texture on phase formation is also clear when comparing the phase formation sequence for a thin $\mathrm{Ni}$ film on $\mathrm{Si}(001)$ and $\mathrm{Si}(111)$ substrates. A detailed comparison between the formation sequences on both substrates was published by Gaudet et al. in 2011 (Ref. 33) and revealed a significant difference in the formation sequence prior to the formation of the technologically relevant axiotaxial $\mathrm{NiSi}$. In contrast to the mainly fiber-textured phases formed on $\mathrm{Si}(001)$ (see discussion above), the phase formation on $\mathrm{Si}(111)$ is dominated mainly by epitaxially textured phases. The as-deposited state is characterized by a mixture of epitaxial, fiber, and random $\mathrm{Ni}$ grains together with a very thin layer of epitaxial $\theta$ (see previous paragraph). Upon annealing, the reaction proceeds with the formation of epitaxial $\delta$ $\mathrm{Ni}_{2} \mathrm{Si}$ and a so far unknown epitaxial silicide, followed by epitaxial $\mathrm{Ni}_{3} \mathrm{Si}_{2}$ which then transforms into NiSi with the axiotaxy texture described in Section III D 3. ${ }^{33}$ The different $\delta$ - $\mathrm{Ni}_{2} \mathrm{Si}$ texture (epitaxy vs fiber) on $\mathrm{Si}(111)$ clearly influences the rest of the phase formation sequence as $\mathrm{Ni}_{3} \mathrm{Si}_{2}$ is formed on $\mathrm{Si}(111)$ instead of the $\theta$ phase on $\mathrm{Si}(001)$. Gaudet et al. suggested different thermodynamic and kinetic explanations as to why the $\theta$ phase forms on $\mathrm{Si}(001)$ while $\mathrm{Ni}_{3} \mathrm{Si}_{2}$ forms on $\mathrm{Si}(111)$. For more details, the reader is referred to Ref. 33.

Very recently, we have obtained similar results in the phase formation sequence of a thin Ni film on Ge. Pole figure measurements at different stages during the reaction towards $\mathrm{NiGe}$ have revealed the presence of a similar transient hexagonal, metastable germanide, i.e., $\epsilon-\mathrm{Ni}_{5} \mathrm{Ge}_{3}$, forming epitaxially before the formation of $\mathrm{NiGe}$ on both $\mathrm{Ge}(001)$ and $\mathrm{Ge}(111)$ substrates. ${ }^{121}$

From the discussion above, it is clear that texture measurements at different stages during a solid-state reaction can 
provide valuable information in order to identify the phase(s) present at different temperatures during the formation sequence, especially when the forming phases are heavily textured. Furthermore, the orientation of the single crystalline substrate has a strong effect on the texture of the different phases in the formation sequence, leading to different interfaces and interface energies for different substrate orientations. This can affect the phase formation sequence by changing the formation temperature of a phase or by having extra phases forming or some not appearing depending on the substrate orientation.

\section{B. Influence on phase stability}

While the discussion in Subsection IV A was focussed on how texture can influence the phase formation sequence during a solid-state reaction between a thin metal film and silicon or germanium, this subsection will handle the influence of texture on the phase stability of one specific phase. With the term "phase stability," we refer to the maximum temperature up until which an intermediate phase is stable before it reacts with the substrate to form a phase that is more thermodynamically stable in contact with the pure $\mathrm{Si}$ or Ge substrate. To illustrate this point, we will discuss the influence of texture on the stability of $\mathrm{Co}_{5} \mathrm{Ge}_{7}$, a germanide that forms during the solid-state reaction between a thin Co film and a $\mathrm{Ge}$ substrate. The complete phase formation sequence for a $30 \mathrm{~nm}$ Co film on a $\mathrm{Ge}(001)$ substrate was established by Gaudet et al. ${ }^{23}$ in 2006 as being $\mathrm{Co} \rightarrow \mathrm{CoGe}$ $\rightarrow \mathrm{Co}_{5} \mathrm{Ge}_{7} \rightarrow \mathrm{CoGe}_{2}$.

In 2010, De Keyser et al. published a detailed comparison between phase formation of a $30 \mathrm{~nm}$ Co film on $\mathrm{Ge}(001)$ and (111) oriented substrates. ${ }^{42}$ Using in situ XRD, they studied the phase formation sequence on both substrates (see Fig. 18). On $\mathrm{Ge}(001)$, the phase formation sequence observed by Gaudet $e t a l{ }^{23}$ was reproduced, which is clearly visible in the in situ XRD measurement in Fig. 18(a). However, on $\mathrm{Ge}(111)$, only the final $\mathrm{CoGe}_{2}$ phase could be observed in the in situ XRD measurement, albeit characterized by different relative intensities for the diffraction peaks as on $\mathrm{Ge}(001)$, and no diffraction peaks could be observed at lower temperatures. More importantly, the formation temperature of $\mathrm{CoGe}_{2}$, when formed using a ramp anneal at $3{ }^{\circ} \mathrm{C} / \mathrm{s}$, is about $30^{\circ} \mathrm{C}$ higher on $\mathrm{Ge}(111)$.

As the difference in relative intensities of the $\mathrm{CoGe}_{2}$ diffraction peaks on both substrate types and the absence of diffraction peaks at lower temperatures on the $\mathrm{Ge}(111)$ substrate are strong indications of a high amount of texturing in the films, De Keyser et al. used pole figure and EBSD measurements to study the texture of the formed films at two different temperatures. A first quench was taken at $725^{\circ} \mathrm{C}$ where $\mathrm{CoGe}_{2}$ is visible on both substrates, and a second quench was done before the formation of $\mathrm{CoGe}_{2}$ at $600^{\circ} \mathrm{C}$, where $\mathrm{Co}_{5} \mathrm{Ge}_{7}$ is visible on $\mathrm{Ge}(001)$. These texture measurements revealed that also on $\mathrm{Ge}(111)$ at $600{ }^{\circ} \mathrm{C}$, the film consists of $\mathrm{Co}_{5} \mathrm{Ge}_{7}$. However, this $\mathrm{Co}_{5} \mathrm{Ge}_{7}$ exhibits a strong epitaxial alignment for which the $\mathrm{Co}_{5} \mathrm{Ge}_{7}(0 \overline{2} 1)$ plane is parallel to the substrate and the $\mathrm{Co}_{5} \mathrm{Ge}_{7}(100)$ plane is parallel to $\mathrm{Ge}(01 \overline{1})$. When further annealing this film to $725^{\circ} \mathrm{C}$, De
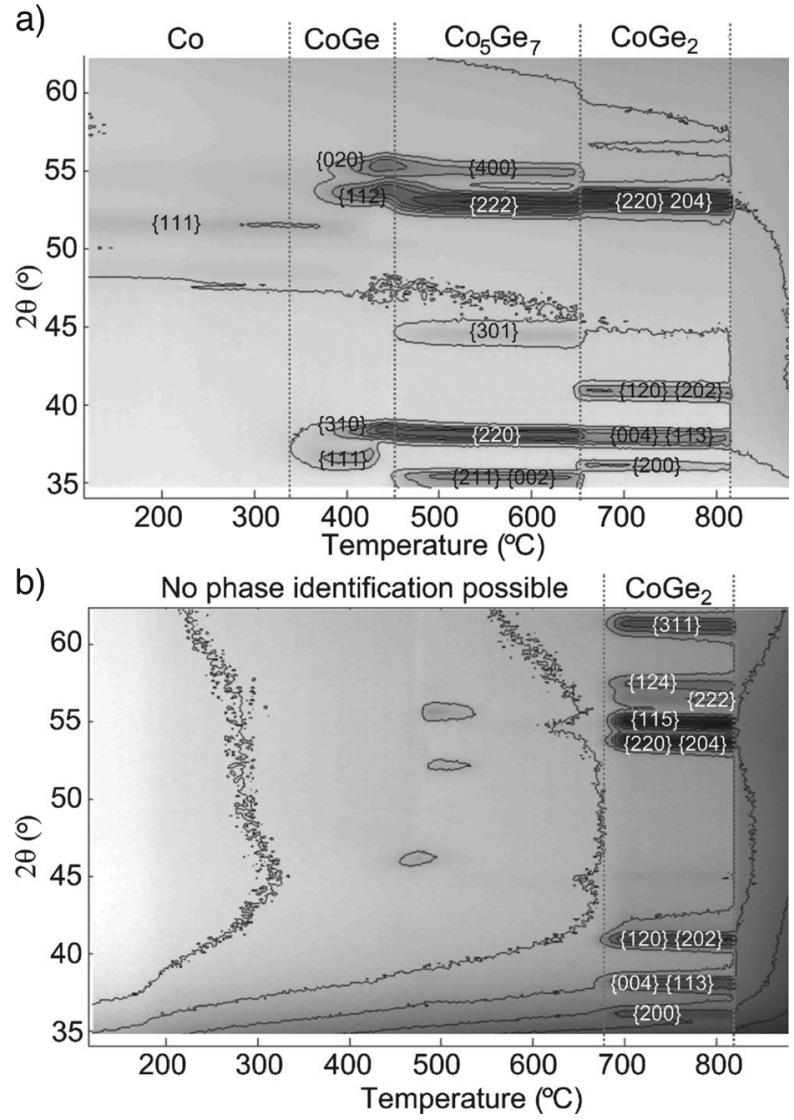

FIG. 18. In situ XRD measurement for a $30 \mathrm{~nm}$ Co film on (a) $\mathrm{Ge}(001)$ and (b) $\mathrm{Ge}(111)$. Darker areas indicate higher diffracted intensity. The heating rate during the measurements was $3{ }^{\circ} \mathrm{C} / \mathrm{s}$. Reproduced with permission from De Keyser et al., J. Electrochem. Soc. 157, H395 (2010). Copyright 2010 The Electrochemical Society.

Keyser et al. observed the presence of $\mathrm{CoGe}_{2}$ which is mostly randomly oriented, along with some remaining epitaxial $\mathrm{Co}_{5} \mathrm{Ge}_{7}$. This formation of strongly epitaxial $\mathrm{Co}_{5} \mathrm{Ge}_{7}$ and the simultaneous presence of $\mathrm{Co}_{5} \mathrm{Ge}_{7}$ and $\mathrm{CoGe}_{2}$ on $\mathrm{Ge}(111)$ is in sharp contrast to the phase formation sequence observed on $\mathrm{Ge}(001)$, where $\mathrm{Co}_{5} \mathrm{Ge}_{7}$ was observed to exhibit a complex texture with three epitaxy and two axiotaxy components. ${ }^{42}$ Further annealing to $725^{\circ} \mathrm{C}$ on $\mathrm{Ge}(001)$ led to the formation of textured $\mathrm{CoGe}_{2}$ exhibiting five different epitaxy and three axiotaxy components. No simultaneous presence of $\mathrm{Co}_{5} \mathrm{Ge}_{7}$ and $\mathrm{CoGe}_{2}$ was observed for the reaction on $\mathrm{Ge}(001)$.

According to De Keyser et al., the improved phase stability of $\mathrm{Co}_{5} \mathrm{Ge}_{7}$ and thus the increased formation temperature of $\mathrm{CoGe}_{2}$ on $\mathrm{Ge}(111)$ can be attributed to the strong epitaxial orientation of $\mathrm{Co}_{5} \mathrm{Ge}_{7}$ on this substrate as the lower interface energy of the epitaxial grains (compared to randomly oriented grains) leads to a lower driving force for the conversion to $\mathrm{CoGe}_{2}$, shifting the $\mathrm{CoGe}_{2}$ formation to higher temperatures. This explanation is corroborated by the microstructure of the films, which was measured using EBSD. In Fig. 19, EBSD maps performed on both substrates are shown for the same quenching temperatures as for the pole figure measurements. For $\mathrm{Co}_{5} \mathrm{Ge}_{7}$ on $\mathrm{Ge}(111)$ (top left EBSD map in Fig. 19), the microstructure seems to consist of a few larger grains embedded in a background of 

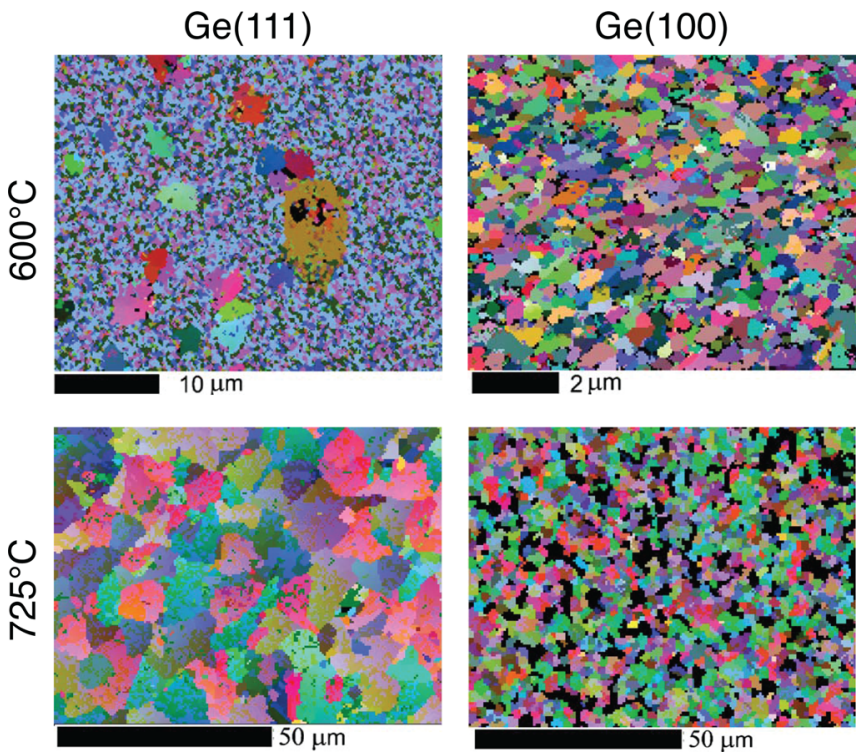

FIG. 19. EBSD maps of $30 \mathrm{~nm}$ Co films deposited on Ge(001) and Ge(111), quenched at $600{ }^{\circ} \mathrm{C}$ and $725^{\circ} \mathrm{C}$. Reproduced with permission from De Keyser et al., J. Electrochem. Soc. 157, H395 (2010). Copyright 2010 The Electrochemical Society.

seemingly small grains. However, detailed analysis has shown that these seemingly small grains all belong to the same epitaxial $\mathrm{Co}_{5} \mathrm{Ge}_{7}$ orientation discussed above (rotationally equivalent orientations were indexed differently by the EBSD software) and that the average $\mathrm{Co}_{5} \mathrm{Ge}_{7}$ grain size is as large as $5 \mu \mathrm{m}$. In contrast, the microstructure of $\mathrm{Co}_{5} \mathrm{Ge}_{7}$ on $\mathrm{Ge}(001)$ (top right EBSD map in Fig. 19) shows a typical polycrystalline film with an average grain size of $250 \mathrm{~nm}$, about 20 times smaller compared to $\mathrm{Ge}(111)$. The large $\mathrm{Co}_{5} \mathrm{Ge}_{7}$ grain size on $\mathrm{Ge}(111)$ leads to a much lower density of nucleation sites (triple and quadruple grain boundary points) for the nucleation controlled transformation of $\mathrm{Co}_{5} \mathrm{Ge}_{7}$ to $\mathrm{CoGe}_{2}$ compared to the $\mathrm{Ge}(100)$ substrate. As a result, the nucleation rate for $\mathrm{CoGe}_{2}$ is much lower on $\mathrm{Ge}(111)$, spreading the conversion from $\mathrm{Co}_{5} \mathrm{Ge}_{7}$ to $\mathrm{CoGe}_{2}$ over a much longer time which results in $\mathrm{Co}_{5} \mathrm{Ge}_{7}$ being present at higher temperatures on $\mathrm{Ge}(111)$ compared to $\mathrm{Ge}(001)$ and consequently in the simultaneous observation of both phases on $\mathrm{Ge}(111) .^{42}$ Furthermore, the low density of $\mathrm{CoGe}_{2}$ nucleation centers available on $\mathrm{Ge}(111)$ also leads to large $\mathrm{CoGe}_{2}$ grains on $\mathrm{Ge}(111)$ (see bottom left EBSD map in Fig. 19) as the nuclei can grow larger before encountering a competing $\mathrm{CoGe}_{2}$ grain. ${ }^{42}$

Clearly, texture can have a profound influence on phase stability during silicide/germanide formation. The low interface energy which is typical for a phase exhibiting a strong epitaxial orientation will result in a larger change in interface energy for the transformation to the next phase compared to the case where the phase has a less pronounced preferential orientation. As the activation energy for the transformation greatly depends on the change in interface energy ${ }^{106}$ $\left(\Delta G^{*} \sim(\Delta \sigma)^{3} /(\Delta H)^{2}\right)$, the activation energy for the transformation of the epitaxially textured phase will be higher, resulting in an increase of the formation temperature during a ramp anneal. Recently, we have observed similar results as discussed above for $\mathrm{Co}-\mathrm{Ge}$ during the solid-state reaction between $30 \mathrm{~nm}$ Pt films with $\mathrm{Ge}(001)$ and $\mathrm{Ge}(111)$. Pole figure measurements performed on different quenches during the reaction revealed strong epitaxial alignments of $\mathrm{Pt}_{2} \mathrm{Ge}$, PtGe and $\mathrm{PtGe}_{2}$ on $\mathrm{Ge}(111)$, while these phases are observed to be mostly randomly textured on $\mathrm{Ge}(001)$. The phase stability of the epitaxial phases on $\mathrm{Ge}(111)$ was found to be enhanced, leading to generally higher formation temperatures of the different platinum germanides on a $\mathrm{Ge}(111)$ substrate compared to a $\mathrm{Ge}(001)$ substrate.

\section{Influence on morphological stability}

Once a specific silicide/germanide phase has been formed to serve as an electrical contact to the source, drain, and possibly the gate of a MOSFET device, it has to remain stable and not degrade during the subsequent CMOS fabrication processing steps. For NiSi, there are two important degradation mechanisms during temperature treatments. First, since $\mathrm{NiSi}_{2}$, not $\mathrm{NiSi}$, is the end phase in the solid-state reaction between a thin $\mathrm{Ni}$ film and a Si substrate, further heating of the NiSi contact could lead to the formation of the undesired, high resistive $\mathrm{NiSi}_{2}$ phase. This relates to the "phase stability" that was discussed in Sec. IV B for the case of $\mathrm{Co}_{5} \mathrm{Ge}_{7}$. Second, heating a NiSi film can lead to agglomeration, i.e., the breaking up of the film into small islands. The agglomerated film then consists of small islands of lowresistive NiSi embedded in a background of high-resistive silicon, destroying the low resistivity of the contact.

A detailed study of these two degradation mechanisms of NiSi films was published in 2005 by Deduytsche et al. ${ }^{110}$ Their observations led to two important conclusions. First, for Ni films with thicknesses relevant for contact formation in CMOS devices $(<15 \mathrm{~nm})$, agglomeration of the film occurs at much lower temperatures than the conversion to $\mathrm{NiSi}_{2}$, making agglomeration the main degradation mechanism for such films. Second, it was found that agglomeration occurs more quickly on a single crystalline (001) oriented SOI (Silicon On Insulator) substrate compared to a polycrystalline Si substrate. This second observation is illustrated in Fig. 20, where the morphological stability of different NiSi lines, formed on poly-Si gate and SOI source/drain regions and subjected to the same thermal budget, is visible. It can be seen that the NiSi films on top of the single-crystalline source/drain regions are severely agglomerated while the films on top of the poly-Si gate regions are still nicely continuous. This result was very surprising, as previous studies of the morphological stability of thin silicide films systematically showed a better stability on single-crystal substrates. ${ }^{122}$ Indeed, a typical process to stabilize a film on a polycrystalline $\mathrm{Si}$ substrate is to anneal the substrate prior to metal deposition. This anneal results in an increase of the grain size in the polycrystalline substrate, improving the morphological stability of the film. Hence, a film formed on a single crystalline substrate (which is basically one big grain) would be expected to exhibit the largest morphological stability. Although not backed by experimental evidence, Deduytsche et al. suggested the axiotaxial texture of NiSi on single- 


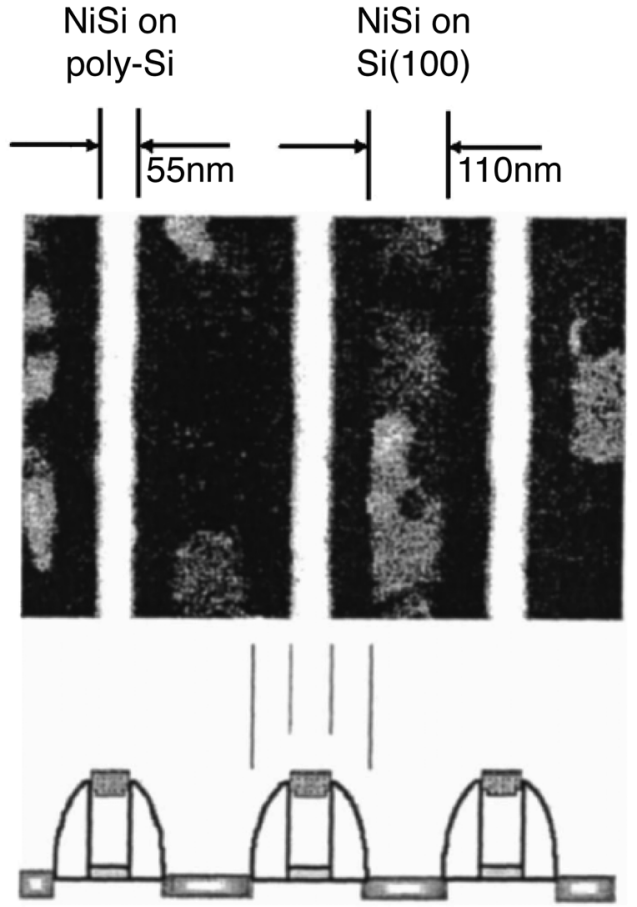

FIG. 20. Top-view SEM picture of NiSi lines formed on the poly-Si gate and SOI source/drain regions of traditional MOSFET device structures. While subjected to the same thermal budget, the NiSi lines on the single-crystalline SOI regions are already agglomerated, while the lines on the poly-Si gate regions are still continuous. Figure reprinted with permission from J. Appl. Phys. 98, 033526 (2005). Copyright 2005 AIP Publishing LLC. crystalline $\mathrm{Si}$ substrates as a possible explanation for the enhanced agglomeration on a single crystalline substrate. ${ }^{110}$

In 2008, De Keyser et al. published a study focussing on the texture evolution of a thin NiSi film during agglomeration in an attempt to understand the link between the NiSi axiotaxy texture and the agglomeration behavior on a single crystal $\mathrm{Si}(001)$ substrate. ${ }^{123}$ To this end, XRD pole figures were recorded on $20 \mathrm{~nm} \mathrm{NiSi}$ films formed on $\mathrm{Si}(001)$ that were quenched at different temperatures. These NiSi(112) pole figures are visible in the rightmost column of Fig. 21 along with SEM and TEM pictures showing the morphology of the films at the quenched temperatures. It is clear that for higher temperatures and thus more severe stages of agglomeration (as evidenced by the SEM/TEM pictures), the axiotaxy lines increase in intensity and the background coming from randomly oriented grains decreases significantly. De Keyser et al. quantified the increase of axiotaxy in the film by recording the intensity of the (103) off-normal fiber axis pole in the (103) pole figure at the different temperatures. The result is visible in the line graph in Fig. 21 along with the corresponding sheet resistance of the film. From this graph, a clear correlation between the film agglomeration (characterized by the increase in sheet resistance) and the increase of the axiotaxy intensity is observed, suggesting that during agglomeration, randomly oriented grains disappear in favor of axiotaxial grains.

This raises the question of how the presence of axiotaxy renders a NiSi film more prone to agglomeration. Why does the intensity of the axiotaxy lines increase during the agglomeration process? The driving force for the
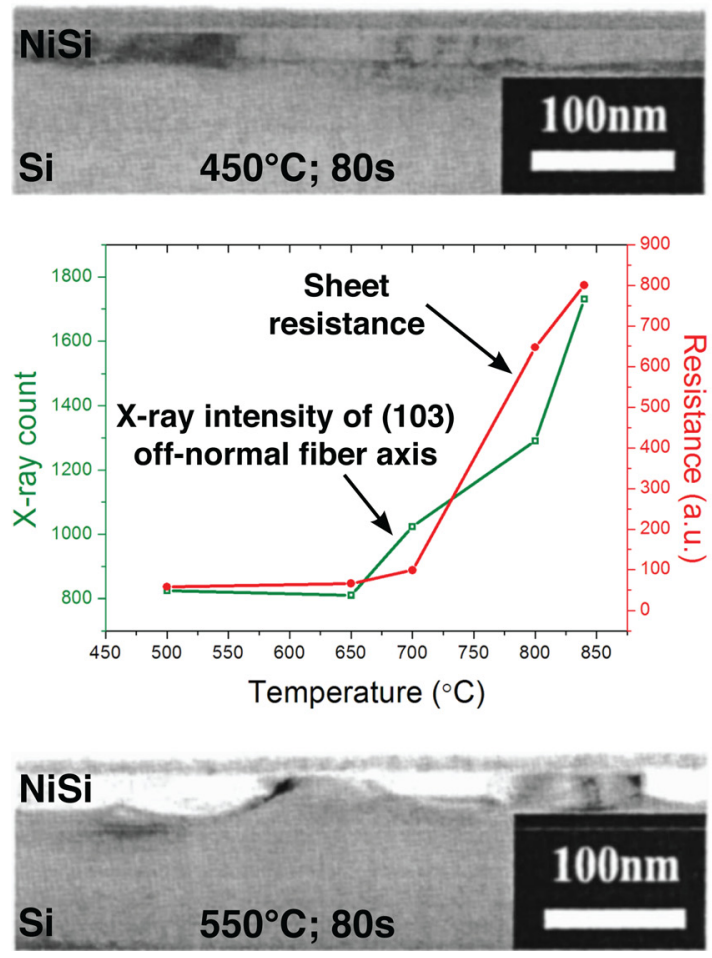
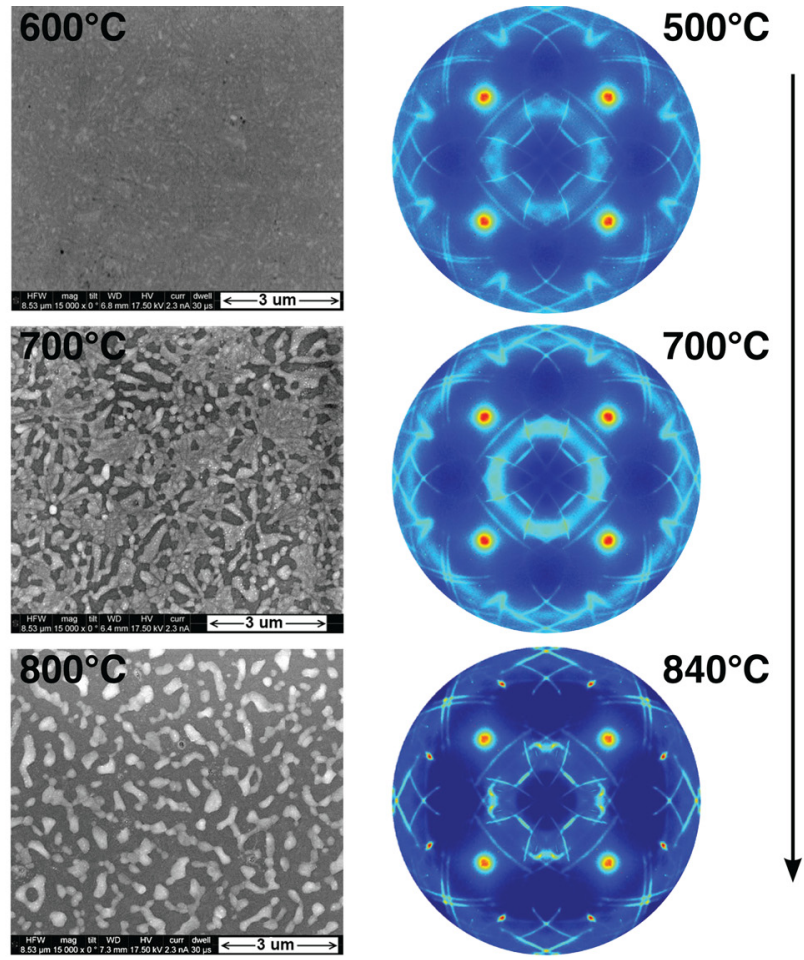

FIG. 21. TEM (left column), SEM (middle column) and pole figure (right column) measurements for a thin Ni film on Si(001) at different temperatures representing different agglomeration stages. The samples for the SEM and pole figure measurements were quenched at the indicated temperatures using an anneal at a rate of $3{ }^{\circ} \mathrm{C} / \mathrm{s}$. The samples for the TEM measurements were annealed during 1980s at the indicated temperatures. The line graph in the left column shows the X-ray intensity of the (103) off-normal fiber axis -as obtained from the NiSi(103) pole figures taken on samples quenched at the different temperaturesalong with the sheet resistance of the corresponding NiSi films. Agglomeration is characterized by the rise in the sheet resistance values. 
agglomeration of a silicide/germanide film is considered to be a reduction of the surface and interface energy. ${ }^{122,124}$ However, from the TEM cross-section visible in the bottom left of Fig. 21, it seems that for an agglomerated NiSi film, only the silicide/silicon interface is severely roughened while the surface remains flat, suggesting that minimizing the interface energy is the main driving force for the agglomeration. This interface energy can roughly be calculated as

$$
E \sim \sum_{i} \gamma_{i} A_{i}
$$

where the sum runs over all grains in the film, with $\gamma_{i}$ the interface (and grain boundary) energy per unit area and $A_{i}$ the interface (and grain boundary) area for grain $i$. Taking Eq. (4) into account, one can imagine that the drive for interface energy minimization will result in an abnormal grain growth promoting those grains with a low interface energy that are able to obtain a curved (spherical) interface with the substrate (as this amounts to the smallest interface area). This process is illustrated in Fig. 22, showing a simplified evolution of $\mathrm{NiSi}$ grains on $\mathrm{Si}(001)$ belonging to different texture components. High energy interfaces are colored in red, while the medium (axiotaxy) and low (epitaxy) energy interfaces are indicated by dashed or full green lines, respectively. Typically, grain growth in a single phase is assumed to happen through a process called grain boundary grooving, ${ }^{39,124}$ where mass transport occurs from the high energy grain boundaries towards the grain/substrate interface of the grain with the lowest interface energy, resulting in a grooving of the grain boundary. This would imply that randomly oriented grains will be consumed by epitaxial and axiotaxial grains which have a significantly lower interface energy (Fig. 22(b)). However, the two-dimensional matching at the interface

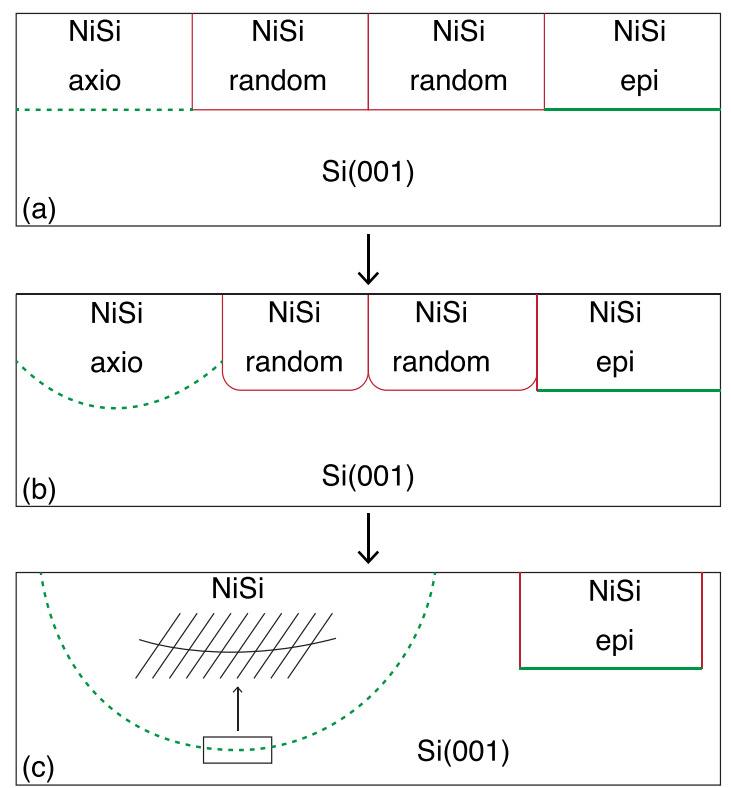

FIG. 22. Illustration of the abnormal grain growth occurring in thin NiSi films on $\mathrm{Si}(001)$ for an increasing thermal budget. High energy interfaces are colored in red, and medium (for axiotaxy) and low (for epitaxy) energy interfaces are represented by a dashed green or a full green line, respectively. between an epitaxial grain and the substrate would be destroyed by curving the interface, rendering a high energy interface. Thus, grain boundary grooving for an epitaxial grain will be difficult, which suppresses the growth of these grains. This explains why highly epitaxial films are typically observed to be very stable with respect to agglomeration. For axiotaxy grains on the other hand, the plane alignment and thus the one-dimensional periodicity in the interface plane is preserved irrespective of the curvature of the interface (see Section III C). This means that the axiotaxy grains can easily grow and form a curved interface, thus minimizing the interface area, and maintain their low interface energy at the same time (see Fig. 22(c)). According to the model described above, the presence of axiotaxy should thus render a thin silicide/germanide film more prone to agglomeration.

Clearly, texture has a defining influence on the morphological stability of a thin NiSi film. As agglomeration was found to be the primary degradation mechanism for NiSi contacts, the development of methods that improve the morphological stability of $\mathrm{NiSi}$ are crucial for the fabrication of reliable microelectronic devices. In view of the discussion in this section, these methods should first aim at reducing the axiotaxy in these thin NiSi films. A widespread approach to improve the stability of NiSi contacts is to alloy the nickel film with additional elements. In particular, the addition of a few at. \% of Pt to the nickel layer results in an improved morphological stability. ${ }^{118}$ Furthermore, the addition of Pt has the added advantage that the phase stability of $\mathrm{NiSi}$ is also improved (by shifting the nucleation of $\mathrm{NiSi}_{2}$ to higher temperatures), ${ }^{19}$ thus tackling the second degradation mechanism. Details on how the addition of alloying elements can influence the texture and hence the morphological stability of NiSi (and other silicide) films will be discussed in Section V C.

\section{Influence on electrical properties}

As silicides and germanides are commonly used in the microelectronics industry as electrical contacts to the source and drain regions of MOSFET devices, a clear understanding of the interface properties of a silicide/Si or germanide/Ge contact is very important. The intrinsic resistance of an interface between a metal and a semiconductor depends primarily on the dopant concentration within the semiconductor and the Schottky barrier height $(\mathrm{SBH})$ between the metal and the semiconductor. This SBH represents the potential energy barrier for majority charge carriers that flow between the silicide and the semiconductor substrate. Due to its importance, a lot of research, both covering SBH determination of different metal-semiconductor (MS) contacts and theoretical modeling of SBH formation, has been published since the 1950s. The interested reader is referred to the excellent reviews by Tung on this subject. ${ }^{125,126}$

In its simplest form (the Schottky-Mott rule ${ }^{127,128}$ ), the SBH for a metal-semiconductor contact is defined as the difference between the metal work function $\left(\Phi_{M}\right)$ and the semiconductor electron affinity $\left(\chi_{S C}\right)$, i.e., $\Phi_{B, n}^{0}=\Phi_{M}-\chi_{S C}$ for an n-type semiconductor. This simple description only holds in the absence of any interaction between the metal and the semiconductor (like charge rearrangement and atomic 
relaxation) at the MS interface, which is not sufficient to describe "real" MS interfaces. Over the past decades, research performed on SBH formation showed a clear dependence of the SBH on the local interface structure of the MS contact. Therefore, a so-called interface dipole term is added to the model which must account for the deviation of the observed SBH from the simple Schottky-Mott SBH, thus modeling the interface chemistry at the MS interface. However, the calculation of this interface dipole term is not straightforward. ${ }^{126}$

As the SBH seems to depend greatly on the local structure of the MS interface, an influence of the texture of a silicide/germanide contact to $\mathrm{Si}$ or $\mathrm{Ge}$ on the $\mathrm{SBH}$ can be expected since the structure of the interface is naturally dependent on the local orientation of the silicide grains. This was experimentally observed in different studies during the 1980s and 1990s when SBH formation was studied for epitaxial $\mathrm{NiSi}_{2}$ contacts on $\mathrm{Si}(111)$ and (001). ${ }^{129-133}$ Such studies were very important in the field of SBH research at first because it was believed that the SBH was the result of a difference between bulk properties of the metal and the semiconductor and as such should not depend on the crystal orientation. Therefore, the single-crystal epitaxial $\mathrm{NiSi}_{2}$ films were ideal model systems for SBH formation studies because of the sharpness and the homogeneous atomic structure of such an epitaxial interface. For an epitaxial $\mathrm{NiSi}_{2}$ film on $\mathrm{Si}(111)$, there are two possible grain orientations, called type A and type B, respectively. Type A has the same orientation as the Si substrate while the type B orientation shares the $\mathrm{Si}(111)$ surface normal but is rotated over $180^{\circ}$ around this axis. Different studies have shown that type B oriented $\mathrm{NiSi}_{2}$ films show a distinctively higher SBH than type A oriented films on n-type $\mathrm{Si}(111)$ (difference of about $0.14 \mathrm{eV}$ ). ${ }^{129-132}$ Furthermore, epitaxial $\mathrm{NiSi}_{2}$ films on n-type $\mathrm{Si}(001)$ were observed to have a SBH that was about $0.40 \mathrm{eV}$ lower than the type $\mathrm{B} \mathrm{NiSi}_{2}$ films on n-type $\mathrm{Si}(111) .{ }^{133}$

A straightforward consequence of the dependence of the SBH on the local interface structure would be that a polycrystalline MS-contact exhibits a spatially inhomogeneous $\mathrm{SBH}$. This would mean that the macroscopically measured value of the SBH for an entire MS-contact is just an average among a significant range of local SBH's spatially distributed over the contact. This idea of SBH inhomogeneity was introduced during the 1980s when it was found that assuming a distribution of SBH's in a contact instead of a single SBH could explain observed differences in the SBH value obtained from I-V measurements versus $\mathrm{C}-\mathrm{V}$ measurements. ${ }^{134}$ From then on, developments in SBH formation models consequently included the idea of SBH inhomogeneity. ${ }^{125,126,135-137}$ With the development of Ballistic Electron Emission Microscopy (BEEM) in the late 1980s, ${ }^{138}$ an experimental technique became available that was able to locally measure Schottky barriers and thus spatially map SBH variations on the nanometer scale. Consequently, a range of studies were performed during the 1990s where direct proof of SBH inhomogeneity was observed on different kinds of MS interfaces, e.g., for epitaxial $\mathrm{CoSi}_{2}$ films ${ }^{139-142}$ and $\mathrm{Au} / \mathrm{n}-\mathrm{Si}$ contacts. ${ }^{143} \mathrm{~A}$ detailed overview of these studies can be found in the excellent reviews by Tung. ${ }^{125,126}$ In the case of silicides, a
BEEM study on polycrystalline $\mathrm{CoSi}_{2}$ contacts formed on ntype $\mathrm{Si}(001)$ was performed by $\mathrm{Zhu}$ et al. ${ }^{144}$ who found an agreement between the SBH distribution obtained by either spatially mapping SBH variations using BEEM or using standard I-V/C-V measurements.

\section{FACTORS AFFECTING TEXTURE DURING SOLID STATE REACTIONS}

The discussions in Sec. IV clearly show that texture can have an important influence on some technologically very relevant properties of thin film silicides/germanides. As a result, film texture in a small contact will affect the electrical properties of a device. Therefore, the ability to control or influence the texture of silicides and germanides during contact formation could enable us to tune and optimize important contact properties. In this section, we aim to give an overview of different studies that investigated the influence of selected experimental parameters/techniques on the texture evolution of the desired silicide/germanide phase. As these studies typically focus on a single parameter, it must be noted that in reality, the final texture is determined by the interplay of different factors. This is what makes the development of a predictive theory for texture formation not straightforward. As was mentioned in the introductory chapter, the results obtained so far (which are discussed below) are not yet sufficient for such a theory to be developed.

\section{A. Dopants}

As silicide contact formation in CMOS technology takes place on the doped source and drain regions of the Si substrate, it is important to study the effects of dopant type and concentration as well as dopant redistribution during the solid-state reaction on the properties of the final silicide contact. ${ }^{145-147}$ In this view, studying the influence of this dopant redistribution on the texture of the formed silicide phases is directly relevant. Nonetheless, literature reports that are concerned with the effect of substrate doping on silicide texture are scarce. This is probably a consequence of the complexity of the phenomenon, since a number of factors are at play such as dopant diffusivity, dopant solubility in both the silicon and the silicide, interface and surface segregation coefficients, and evaporative or reactive dopant losses during the heat-treatment. ${ }^{146}$ Moreover, because of the nature of diffusion and its dependence on available paths such as grain boundaries and interfaces, detailed studies of these redistribution processes require specialized experimental techniques, e.g., Atom Probe Tomography (APT) ${ }^{148-150}$ which allow for full three-dimensional elemental mapping.

Such dopant redistribution studies have been performed by a number of groups. It has been observed that for some dopant-silicide combinations the dopant species accumulate at the silicide/silicon interface, ${ }^{146,147}$ an observation that is referred to as the "snowplow" effect in literature and that has been observed, e.g., in the case of boron redistribution during $\mathrm{NiSi}$ formation ${ }^{150}$ or As redistribution during $\delta-\mathrm{Ni}_{2} \mathrm{Si}$ formation. ${ }^{148}$ In other cases, researchers observed redistribution of the dopants throughout the formed silicide, ${ }^{145,146,148}$ e.g., in the case of As redistribution during the formation of the 
transient $\theta$-Ni-silicide phase, where the As was observed to form clusters within this $\theta$-phase. ${ }^{148}$ Especially in the case where the dopants are accumulated at the interface and thus where the initial low concentration of dopants piles up at the interface as the silicide is growing, an influence on the texture should not come as a surprise.

During the many years of high-resolution, synchrotron based pole figure studies on thin film silicides and germanides at the $\mathrm{X} 20 \mathrm{~A}$ beamline of the national synchrotron lightsource (NSLS) at Brookhaven National Lab (BNL) by the research groups of IBM and Ghent University, multiple measurements have been performed to probe the influence of dopants on silicide texture. Fig. 23 shows a small subset of these measurements. NiSi(112) pole figures are shown for $\mathrm{NiSi}$ formed on single-crystalline silicon-on-insulator (SOI) substrates doped with different elements at comparable doses. The $\mathrm{NiSi}(112)$ pole figure for $\mathrm{NiSi}$ formed on an undoped substrate is provided as a reference at the top of this figure. Different influences on the texture by different dopant species can clearly be observed. From this figure, it is clear that the small concentrations of additional elements can have a drastic impact on the texture of the silicide film. Arsenic shows the mildest influence compared to the undoped Si. For fluorine and boron, a clear reduction in the intensity of the axiotaxy lines can be observed. Phosphorus has the most profound influence on the texture, as the axiotaxy has almost

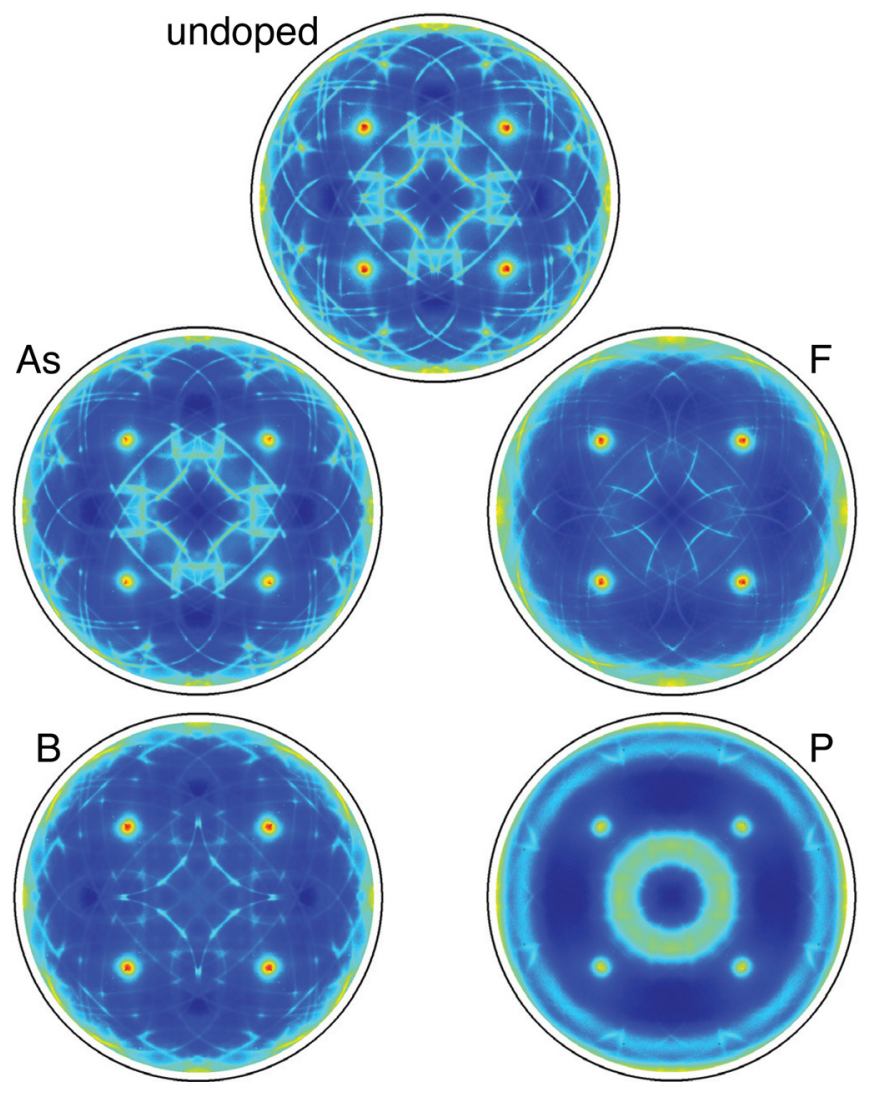

FIG. 23. NiSi(112) pole figures for NiSi formed by annealing $10 \mathrm{~nm} \mathrm{Ni}$ deposited on doped SOI (silicon on insulator) substrates at $500^{\circ} \mathrm{C}$. The dopant concentrations were $8 \times 10^{15} \mathrm{~cm}^{-2}$ for As, B and $\mathrm{P}$ and $3 \times 10^{15} \mathrm{~cm}^{-2}$ for F. The NiSi(112) pole figure for NiSi formed on an undoped SOI substrate is added at the top as a reference. The SOI substrates have a $\mathrm{Si}(001)$ orientation. disappeared while a broad fiber texture develops and can only be detected on this P doped sample. In contrast, Kimura et al. observed the formation of transrotational NiSi (see also Section III D 3) on B-doped $\mathrm{Si}(001)$ substrates with a similar doping dose as was used in our measurements, ${ }^{151}$ suggesting that other experimental conditions are important as well. While variations in substrate and implantation conditions could be a factor here, it is likely that the annealing conditions play the critical role in the observed differences in final texture. As the tooling capabilities evolve, anneals in the industry are now typically done at much faster ramp rates leading to films exhibiting smaller microstructure (because of higher nucleation density). Slower anneals typically lead to larger grain sizes and significant variations in texture.

\section{B. Lattice spacing of the substrate}

For a textured thin silicide/germanide film on a singlecrystal Si or Ge substrate, the driving force for the preferred orientation of the grains is a reduction in either surface energy (fiber texture) or interface energy (epitaxy and axiotaxy). In the latter case, the energy reduction results from an optimized bonding across the interface resulting from a oneor two-dimensional match between the film and substrate lattice in the plane of the interface. Therefore, texture development in such a thin silicide/germanide film should be sensitive to variations in lattice constants (and d-spacings) of either the substrate or the film. In this section, we will discuss an example of how a slight change in the lattice constants of the silicon substrate impacts the texture of $\mathrm{NiSi}$.

In 2011, De Keyser et al. published a study where they investigated the phase formation and texture of NiSi films formed on $\mathrm{Si}(001)$ substrates for which the top $40 \mathrm{~nm}$ consisted of an epitaxial $\mathrm{Si}_{1-x} \mathrm{C}_{x}$ layer containing either 1, 1.7, 2.2 , or 2.5 at. \% of substitutional carbon in the Si lattice. ${ }^{152}$ The much improved morphological stability of NiSi on top of these $\mathrm{Si}_{1-x} \mathrm{C}_{x}$ layers ${ }^{153}$ (from here on, these films will be referred to as $\mathrm{NiSi}(\mathrm{C})$ ) can be explained because the $\mathrm{C}$ atom is known to prevent the diffusion of Si within the film and both elements must be mobile to allow for a modification of the morphology. Here, De Keyser et al. wanted to investigate whether changes in film texture could provide a second explanation for the enhanced morphological stability of these films.

The phase formation sequence towards $\mathrm{NiSi}(\mathrm{C})$ on these $\mathrm{Si}_{1-x} \mathrm{C}_{x}$ substrates was studied by De Keyser et al. using in situ X-ray diffraction. It was observed that, compared to the phase formation sequence on pure $\mathrm{Si}$, the onset of the formation of both the transient Ni-rich phases and the NiSi phase is significantly delayed and that the transient phases are present over a much wider temperature window. Furthermore, for $\mathrm{NiSi}(\mathrm{C})$ formed on $\mathrm{Si}_{1-x} \mathrm{C}_{x}$ samples with a $\mathrm{C}$ content of $2.5 \%$, the intensities of the $\mathrm{NiSi}(\mathrm{C})$ diffraction peaks in the in situ XRD measurements were observed to be significantly different compared to $\mathrm{NiSi}(\mathrm{C})$ formed on $\mathrm{Si}_{1-x} \mathrm{C}_{x}$ samples with $1 \%$ of $\mathrm{C}$ and compared to $\mathrm{NiSi}$ formed on pure $\mathrm{Si}^{34}$ suggesting a change in NiSi texture.

After $\operatorname{NiSi}(\mathrm{C})$ formation and a subsequent hightemperature anneal of $30 \mathrm{~min}$ at $750^{\circ} \mathrm{C}$, De Keyser et al. 
investigated the texture of the resulting $\mathrm{NiSi}(\mathrm{C})$ films using high-resolution pole figures. In Figs. 24(a)-24(c), we reproduced the $\mathrm{NiSi}(\mathrm{C})\{112\}$ pole figures for $\mathrm{NiSi}(\mathrm{C})$ films formed on $\mathrm{Si}_{1-x} \mathrm{C}_{x}$ substrates with $1,1.7$ and 2.2 at. \% of substitutional carbon. For $\mathrm{NiSi}(\mathrm{C})$ formed on the substrates containing more than $1 \%$ of carbon, the typical NiSi axiotaxy lines (see Fig. 15(b)) have disappeared and instead a combination of $\mathrm{NiSi}(\mathrm{C})\{202\},\{211\}$, and $\{112\}$ fiber textures is visible, evidenced by the concentric circles on the pole figures of Figs. 24(b) and 24(c). Furthermore, De Keyser et al. investigated whether only the presence of carbon induces the change in the $\mathrm{NiSi}(\mathrm{C})$ texture or whether the location of the $\mathrm{C}$ atoms is also important. To this end, the texture was investigated for $\mathrm{NiSi}(\mathrm{C})$ formed on a $\mathrm{Si}_{1-x} \mathrm{C}_{x}$ substrate with $2.2 \%$ of $\mathrm{C}$ that was subjected to a spike anneal $\left(\mathrm{He}, 1050^{\circ} \mathrm{C}, 1.5 \mathrm{~s}\right)$ prior to the deposition of $\mathrm{Ni}$. This anneal effectively reduced the concentration of substitutional carbon atoms ( $\mathrm{C}$ atoms that replace $\mathrm{Si}$ atoms in the $\mathrm{Si}$ lattice) from $2.2 \%$ to $0.9 \%$ by moving part of the substitutional C into interstitial sites. The pole figure in Fig. 24(d) shows that in this case the axiotaxy texture is present again, resembling the texture observed for the sample with $1 \% \mathrm{C}$ in the original $\mathrm{Si}_{1-x} \mathrm{C}_{x}$ layer.

The substitution of $\mathrm{Si}$ atoms with $\mathrm{C}$ in the $\mathrm{Si}_{1-x} \mathrm{C}_{x}$ epilayers of the substrate slightly changes the lattice constants with respect to a pristine $\mathrm{Si}$ lattice. As a result, the d-spacing of the $\mathrm{Si}\{220\}$ family of crystal planes will also change when more substitutional $\mathrm{C}$ is present in the substrate. Because the typical NiSi axiotaxy texture results from a matching between $\mathrm{NiSi}\{202\},\{211\},\{103\}$, and $\{112\}$ planes and $\operatorname{Si}\{220\}$ planes at the interface, the change in $\mathrm{Si}\{220\}$ d-spacing due to the presence of substitutional C inhibits the plane matching and thus the axiotaxy texture

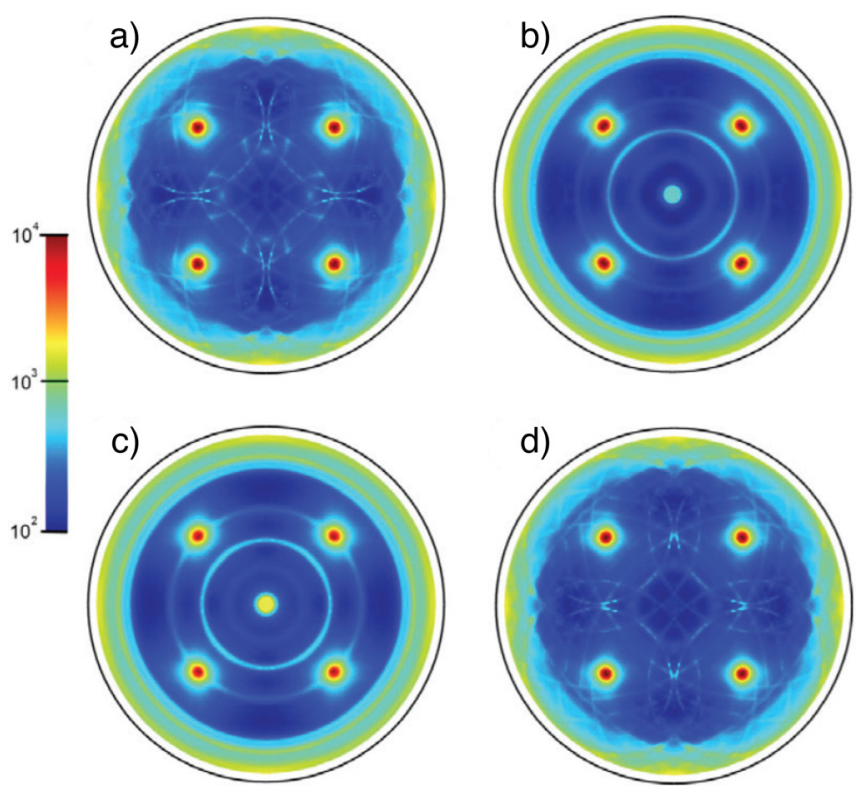

FIG. 24. (a)-(d) $\mathrm{NiSi}(\mathrm{C})\{112\}$ pole figures for $\mathrm{NiSi}(\mathrm{C})$ formed on $\mathrm{Si}_{1-x} \mathrm{C}_{x}$ substrates with different concentrations of $\mathrm{C}$, i.e., (a) with $1 \% \mathrm{C}$, (b) with $1.7 \% \mathrm{C}$, (c) with $2.2 \% \mathrm{C}$, and (d) with $2.2 \% \mathrm{C}$ but where the substrate was subjected to a pre-anneal, reducing the actual substitutional $\mathrm{C}$ concentration to $0.9 \%$. Figure reprinted with permission from De Keyser et al., Microelectron. Eng. 88, 536 (2011). Copyright 2011 Elsevier. when the d-spacing change is large enough (i.e., when more than $1 \%$ of substitutional $\mathrm{C}$ is present). The fact that the axiotaxy texture is present for a substrate where the $2.2 \%$ of substitutional $\mathrm{C}$ is reduced to $0.9 \%$ by spike annealing strengthens the argument that the change in $\mathrm{NiSi}(\mathrm{C})$ texture is due to the change in substrate lattice spacing resulting from the substitutional carbon atoms. Finally, De Keyser et al. argued that the much improved thermal stability of these $\mathrm{NiSi}(\mathrm{C})$ films ${ }^{153}$ can at least be partly explained by the suppression of the axiotaxy texture, because silicide/germanide films that exhibit strong axiotaxy texture are expected to be more prone to agglomeration (see the discussion in Section IV C).

\section{Silicide/germanide alloying with soluble elements}

In Sec. V B, we showed that a change in the lattice constants of the silicon/germanium substrate influences the resulting texture of the film by breaking the condition for axiotaxy. In principle, this condition can also be modified by a change in the silicide/germanide lattice which can be achieved by alloying the targeted silicide/germanide with a small concentration of a soluble element. Here, soluble means that the alloying element can form a silicide that is miscible in the targeted silicide. Practically, the alloying can be accomplished by adding the alloying element to the silicide-forming metal layer during deposition. Besides the expected change in lattice constants caused by incorporating the alloying element into the silicide/germanide lattice, the presence of the alloying element can also influence the kinetics during the formation of the silicide/germanide, which in turn impacts the texture development during the solid-state reaction.

For NiSi contacts that are used in modern sub $100 \mathrm{~nm}$ planar technology nodes, alloying with small concentrations of $\mathrm{Pt}$ is a standard procedure as this significantly improves the thermal and morphological stability of the thin $\mathrm{NiSi}$ film. ${ }^{19}$ As the miscibility of PtSi in NiSi is high due to the similar orthorhombic MnP-type structure of the NiSi lattice and the PtSi lattice, the alloyed Pt gets easily incorporated into the NiSi and a $\mathrm{Ni}_{1-x} \mathrm{Pt}_{x} \mathrm{Si}$ layer is formed. The influence of these small concentrations of $\mathrm{Pt}$ on the texture of the resulting $\mathrm{Ni}_{1_{-}} \mathrm{Pt}_{x} \mathrm{Si}$ film compared to a pristine NiSi layer was investigated in 2004 by Detavernier et al. using synchrotron based pole figure measurements. ${ }^{50}$ In Fig. 25, we reprinted a selection of $\mathrm{NiSi}(112)$ pole figures for $\mathrm{Ni}_{1-x} \mathrm{Pt}_{x} \mathrm{Si}$ films that were formed by reacting $30 \mathrm{~nm}$ layers of a $\mathrm{Ni}(\mathrm{Pt})$ alloy with different $\mathrm{Pt}$ concentrations on $\mathrm{Si}(001)$ at $500^{\circ} \mathrm{C}$ for $30 \mathrm{~s}$. For the lowest Pt concentration of $0.2 \%$, it can be seen that the texture is the same as for a pristine NiSi film formed on $\mathrm{Si}(001)$ (see the $\mathrm{NiSi}(112)$ pole figure in Fig. 15(b) and the description of the NiSi texture in the corresponding section). The overlaid axiotaxy patterns in the top right pole figure for the lowest $\mathrm{Pt}$ concentration represent the calculated axiotaxy lines for the two most intense axiotaxy components (i.e., those for which either $\mathrm{NiSi}\{202\}$ or $\{211\}$ planes are aligned to $\mathrm{Si}\{220\}$ ). As the initial concentration of $\mathrm{Pt}$ in the $\mathrm{Ni}(\mathrm{Pt})$ is increased, a significant change in the texture of the final $\mathrm{Ni}_{1-x} \mathrm{Pt}_{x} \mathrm{Si}$ phase can be observed. The 

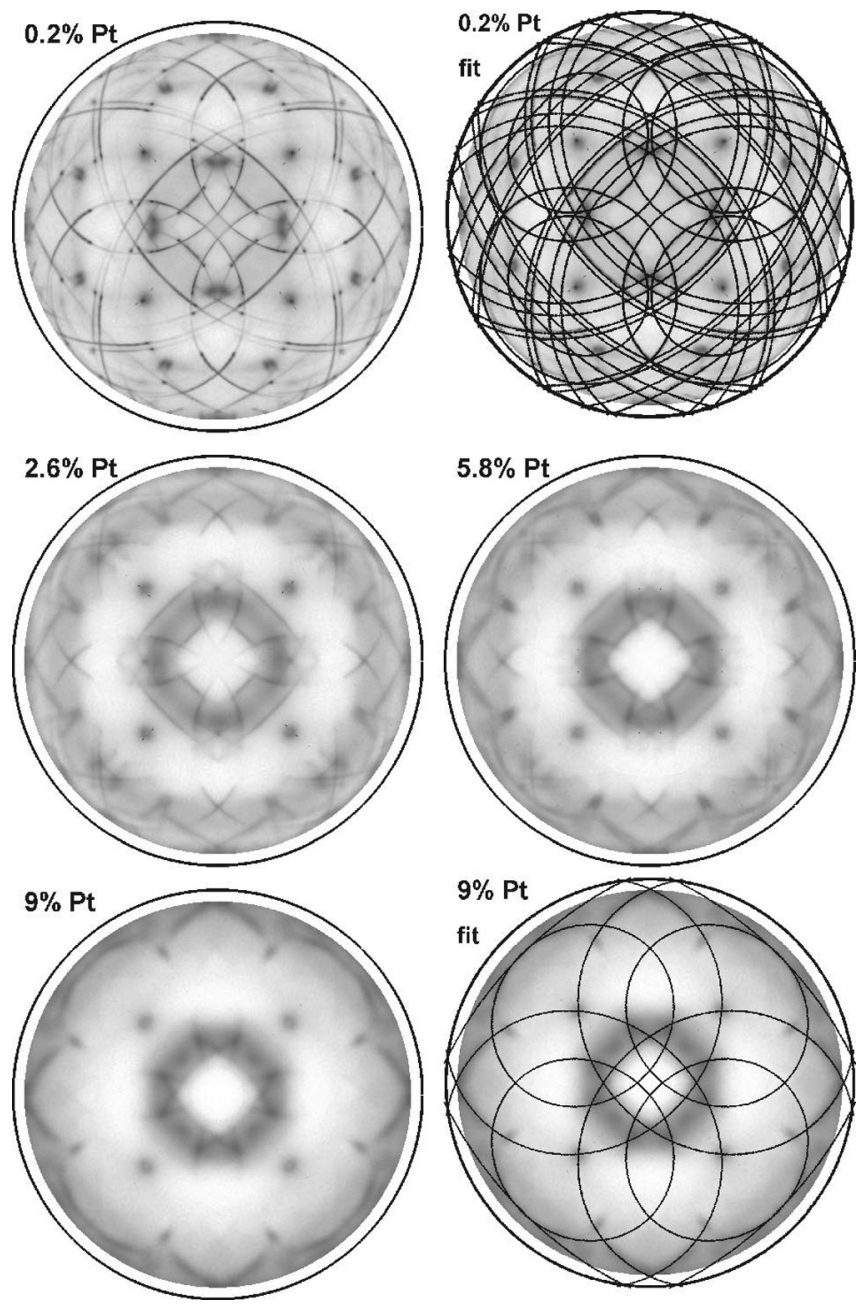

FIG. 25. NiSi(112) pole figure for $\mathrm{Ni}_{1-x} \mathrm{Pt}_{x} \mathrm{Si}$ films formed by the reaction of a $30 \mathrm{~nm} \mathrm{Ni}(\mathrm{Pt})$ alloy with $\mathrm{Si}(001)$ at $500^{\circ} \mathrm{C}$ for $30 \mathrm{~s}$. The concentration of $\mathrm{Pt}$ in the as-deposited alloys is $0.2 \%, 2.6 \%, 5.8 \%$, or $9 \%$. The calculated axiotaxy lines overlaid on the $0.2 \%$ pole figure are for the $\mathrm{NiSi}(202)$ and (211) axiotaxy components, while the overlay on the $9 \% \mathrm{Pt}$ pole figure is for the (103) axiotaxy component. Figure reprinted with permission from Appl. Phys. Lett. 84, 3549 (2004). Copyright 2004 AIP Publishing LLC.

axiotaxy lines for the two most intense NiSi axiotaxy components become weaker and less sharp for increasing Pt concentrations, while the weak $\mathrm{NiSi}\{103\}$ axiotaxy component (see Section III D 3) becomes more prominent, as evidenced by the overlaid pattern in the bottom right pole figure of Fig. 25 which represents the calculated axiotaxy lines for this texture component. The overall reduction of axiotaxy through the alloying with Pt explains, at least partially, the improved morphological stability of the resulting $\mathrm{Ni}_{1-x} \mathrm{Pt}_{x} \mathrm{Si}$ film compared to pure $\mathrm{NiSi}^{19}$ (see Section IV C).

In their paper, Detavernier et al. argued that the change in texture observed for increasing Pt concentrations can be related to the expansion of the $\mathrm{Ni}_{1-x} \mathrm{Pt}_{x} \mathrm{Si}$ unit cell when an increasing amount of $\mathrm{Ni}$ atoms are replaced with Pt. First, this unit cell expansion results in an increase of the dspacing for the $\mathrm{Ni}_{1-x} \mathrm{Pt}_{x} \mathrm{Si}\{202\}$ and $\{211\}$ planes, causing the excellent match with the d-spacing of $\operatorname{Si}\{220\}$ planes to degrade as more $\mathrm{Pt}$ is added, which explains the decrease in intensity and eventually the disappearance of these axiotaxy components. A similar argument holds for the already weak
NiSi $\{112\}$ axiotaxy component. Second, the increased dspacing of the $\mathrm{Ni}_{1-x} \mathrm{Pt}_{x} \mathrm{Si}\{103\}$ planes due to the unit cell expansion leads to an improved matching with $\mathrm{Si}\{220\}$ planes, allowing the match of the interplanar spacing at the interface between the $\mathrm{Ni}_{1-x} \mathrm{Pt}_{x} \mathrm{Si}\{103\}$ and the $\mathrm{Si}\{220\}$ planes to be achieved through a smaller tilt angle $\left(2.5^{\circ}\right.$ instead of $4.2^{\circ}$ ) than for a pure NiSi film. Therefore, the (103)-based axiotaxy is achieved more easily and thus becomes more prominent with increasing $\mathrm{Pt}$ content, as observed in the pole figures of Fig. 25.

As the nucleation stage of a new phase fixes the orientation of the grains, the argumentation of Detavernier et al. discussed above requires that $\mathrm{Pt}$ be present at the boundary between the metal-rich silicide and the Si substrate during the early stages of $\mathrm{NiSi}$ formation from a $\mathrm{Ni}(\mathrm{Pt})$ alloy. If $\mathrm{Pt}$ is not present at the interface, it can not be incorporated in the growing NiSi seeds and influence the texture directly. Hence, to understand the influence of the alloyed Pt on the texture development during the solid-state reaction, detailed information on the Pt redistribution during the reaction is crucial.

This $\mathrm{Pt}$ redistribution during $\mathrm{Ni}_{1-x} \mathrm{Pt}_{x} \mathrm{Si}$ formation was extensively investigated by Demeulemeester et al. using real-time Rutherford Backscattering Spectroscopy (RTRBS) ${ }^{154-156}$ With this technique, a $75 \mathrm{~nm}$ thick $\mathrm{Ni}(\mathrm{Pt})$ alloy is deposited on a $\mathrm{Si}(001)$ substrate and annealed at a rate of $2{ }^{\circ} \mathrm{C} / \mathrm{min}$. During the anneal, an RBS spectrum is collected every two minutes using a $2 \mathrm{MeV} \mathrm{He}{ }^{+}$beam which results in an RBS spectrum being collected every $4^{\circ} \mathrm{C}$. Hence, this technique allows to probe elemental depth distribution during the silicidation with a relatively high temperature resolution, which allowed Demeulemeester et al. to perform a thorough study of the $\mathrm{Pt}$ redistribution during $\mathrm{Ni}(\mathrm{Pt}) \mathrm{Si}$ formation. The experiments revealed that at low temperatures, when $\delta-\mathrm{Ni}_{2} \mathrm{Si}$ forms prior to NiSi formation, only a small amount of the $\mathrm{Pt}$ available in the $\mathrm{Ni}(\mathrm{Pt})$ alloy gets incorporated in the growing $\mathrm{Ni}_{2} \mathrm{Si}$ phase. The majority of the Pt piles up at the $\mathrm{Ni} / \mathrm{Ni}_{2} \mathrm{Si}$ interface and thus gets "snowplowed" towards the surface as more $\mathrm{Ni}_{2} \mathrm{Si}$ grows underneath. This situation is illustrated in the second step of Fig. 26. In this figure, important stages of the Pt redistribution process during the silicidation reaction are schematically depicted, along with two RBS spectra extracted from the RT-RBS data at temperatures in the second and third stage. The RBS spectrum extracted from the second stage (black spectrum) clearly shows the enhanced Pt concentration at the $\mathrm{Ni}_{2} \mathrm{Si}$ / $\mathrm{Ni}\left(\mathrm{Pt}\right.$ ) interface ${ }^{154,155}$ (peak marked with " + " in Fig. 26). Around the same time, similar results were obtained by Hoummada et al. ${ }^{157}$ who used standard RBS on $50 \mathrm{~nm}$ $\mathrm{Ni}(5 \% \mathrm{Pt})$ samples on $\mathrm{Si}(001)$ that were subjected to isothermal anneals between 200 and $300^{\circ} \mathrm{C}$ for different durations.

At the onset of the monosilicide formation, the RT-RBS data of Demeulemeester et al. revealed the presence of a very thin $\mathrm{NiSi}$ layer at the $\mathrm{Ni}_{2} \mathrm{Si} / \mathrm{Si}$ interface together with a high concentration of $\mathrm{Pt}$ at the same depth. This high concentration can be observed in the red RBS spectrum of Fig. 26 through the peak in the platinum signal at the lowest energy (marked with "•"). This Pt concentration is high compared to the $\mathrm{Pt}$ concentration in the $\mathrm{Ni}_{2} \mathrm{Si}$ layer above (valley to the 


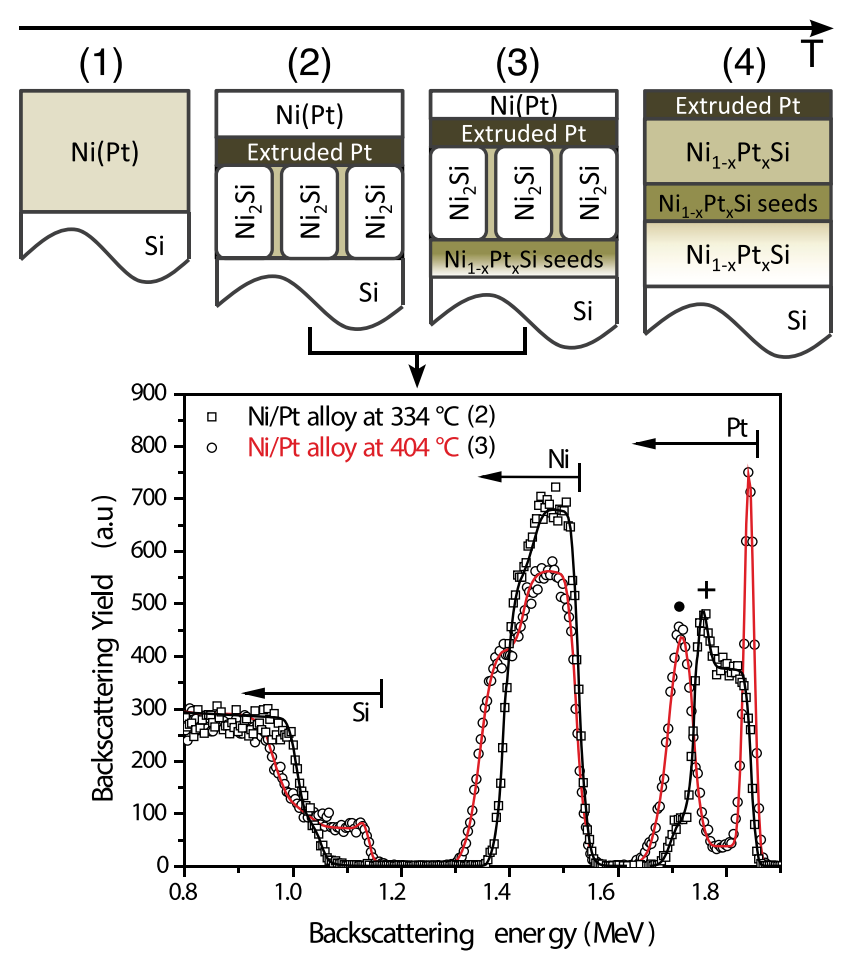

FIG. 26. Top: Schematic summary of several important stages during the formation of $\mathrm{Ni}_{1-x} \mathrm{Pt}_{x} \mathrm{Si}$ from a $\mathrm{Ni}(\mathrm{Pt})$ alloy on $\mathrm{Si}(001)$ as observed by real-time RBS. Bottom: RBS spectra extracted from a real-time RBS measurement performed on a $75 \mathrm{~nm}$ thin $\mathrm{Ni}(7 \% \mathrm{Pt})$ alloy deposited on $\mathrm{Si}(001)$ using a ramp anneal at $2{ }^{\circ} \mathrm{C} / \mathrm{s}$. The spectrum at $334^{\circ} \mathrm{C}$ (open squares) is characteristic of stage 2, i.e., during $\mathrm{Ni}_{2} \mathrm{Si}$ formation, while the spectrum at $404^{\circ} \mathrm{C}$ (open circles) characterizes stage 3 , i.e., during $\mathrm{Ni}_{1-x} \mathrm{Pt}_{x} \mathrm{Si}$ formation. The solid lines represent the RBS simulations. Figures reprinted with permission from J. Appl. Phys. 108, 043505 (2010). Copyright 2010 AIP Publishing LLC.

right of the Pt peak marked with "•") and even slightly exceeds the $\mathrm{Pt}$ concentration in the initial $\mathrm{Ni}(\mathrm{Pt})$ alloy. As a consequence, the initial $\mathrm{Ni}_{1-x} \mathrm{Pt}_{x} \mathrm{Si}$ seeds from which the rest of the phase grows and hence that establish the $\mathrm{Ni}_{1-x} \mathrm{Pt}_{x} \mathrm{Si}$ texture nucleate in the presence of a high $\mathrm{Pt}$ concentration. This observation corroborates the explanation for the texture change put forward by Detavernier et al. that was discussed above.

Next to the real-time RBS studies of Demeulemeester et al., the redistribution of $\mathrm{Pt}$ during the reaction of a $\mathrm{Ni}(\mathrm{Pt})$ alloy with $\mathrm{Si}(001)$ has also been investigated by groups at the university of Aix-Marseille (France) and at the Northwestern University of Illinois (USA) using Atom Probe Tomography (APT). This APT technique allows for a threedimensional reconstruction of the elemental distribution in a small volume, providing both depth and lateral resolution, while RBS only provides depth resolution. However, the APT technique cannot be used in real time during the annealing process. The French group observed that for $50 \mathrm{~nm}$ $\mathrm{Ni}(5 \% \mathrm{Pt})$ films on $\mathrm{Si}(001)$ that were subjected to an isothermal anneal at $300^{\circ} \mathrm{C}$ and quenched during the early growth of the $\mathrm{Ni}_{1-x} \mathrm{Pt}_{x} \mathrm{Si}$ seeds (i.e., before the total consumption of the $\mathrm{Ni}(\mathrm{Pt})$ layer), the $\mathrm{Pt}$ shows a very non-uniform lateral distribution. ${ }^{158}$ It was found that only a limited amount of $\mathrm{Pt}$ is incorporated in the $\mathrm{Ni}_{1-x} \mathrm{Pt}_{x} \mathrm{Si}$ grains, as the majority is located at the $\mathrm{Ni}_{1-x} \mathrm{Pt}_{x} \mathrm{Si}$ grain boundaries and at the $\mathrm{Ni}_{1-x} \mathrm{Pt}_{x} \mathrm{Si} / \mathrm{Ni}_{2} \mathrm{Si}$ interface, while very little $\mathrm{Pt}$ is measured at the $\mathrm{Ni}_{1-x} \mathrm{Pt}_{x} \mathrm{Si} / \mathrm{Si}$ interface. For films with $\geq 10 \%$ of $\mathrm{Pt}$ annealed using isothermals or very slow ramp anneals, the non-stoichiometric $\theta$-phase was found to form epitaxially on $\mathrm{Si}(001)$ (instead of $\delta-\mathrm{Ni}_{2} \mathrm{Si}$ ). ${ }^{159,160}$ In contrast to what was observed for the $\delta-\mathrm{Ni}_{2} \mathrm{Si}$ phase that forms for lower Pt concentrations, the $\mathrm{Pt}$ does get incorporated into the $\theta$-phase in a very non-uniform manner with an enhanced concentration $(\sim 20 \%)$ close to the interface with the unreacted $\mathrm{Ni}(10 \%$ $\mathrm{Pt})$.

The group at Northwestern University studied Pt redistribution in similar $\mathrm{Ni}(5 \% \mathrm{Pt})$ films on $\mathrm{Si}(001)$, but with a thickness of only $10 \mathrm{~nm} .{ }^{161,162}$ After a rapid-thermal anneal at $420^{\circ} \mathrm{C}$ for $5 \mathrm{~s}$, they observed a $5 \mathrm{~nm}$ stoichiometric $\mathrm{NiSi}$ film in contact with the $\mathrm{Si}(001)$ substrate growing outward toward the unreacted $\mathrm{Ni}(5 \%) \mathrm{Pt}$ layer. In these samples, $\mathrm{Pt}$ was observed to have segregated uniformly at the NiSi/ $\mathrm{Si}(001)$ interface. Furthermore, Pt was also observed to be concentrated at the $\mathrm{Ni}(5 \%) \mathrm{Pt} /$ silicide interface and at the $\mathrm{NiSi}$ grain boundaries, indicating a very fast diffusion path along those GBs. ${ }^{161,162}$ They argued that these fast diffusion paths allow the Pt atoms to diffuse rapidly towards the NiSi/ $\mathrm{Si}$ interface during the early stages of NiSi nucleation.

Although both the RT-RBS and the APT results discussed above show the complexity of the Pt redistribution process and its dependency on the initial Pt concentration and the annealing conditions, they do not show the location of the $\mathrm{Pt}$ atoms at the onset of nucleation of the $\mathrm{Ni}_{1-x} \mathrm{Pt}_{x} \mathrm{Si}$ grains, either because lateral resolution is absent in the RTRBS measurements or because the films investigated by APT have already surpassed the initial $\mathrm{Ni}_{1-x} \mathrm{Pt}_{x} \mathrm{Si}$ nucleation stage. As the orientation of these grains and thus the texture of the resulting $\mathrm{Ni}_{1-x} \mathrm{Pt}_{x} \mathrm{Si}$ film is determined at the time of nucleation, Pt distribution experiments focussing on the $\mathrm{Ni}_{1-x} \mathrm{Pt}_{x} \mathrm{Si}$ nucleation stage will be necessary to understand the exact influence of the $\mathrm{Pt}$ atoms on the texture of the resulting $\mathrm{Ni}_{1-x} \mathrm{Pt}_{x} \mathrm{Si}$ film.

The fact that both a change in lattice constants and altered kinetics induced by alloying impact the texture development during the formation of a silicide/germanide has also been observed in the Co-Si system. In 2008, Smeets et al. investigated the influence of alloying increasing amounts of $\mathrm{Ni}$ in a Co film deposited on $\mathrm{Si}(001)$ on the texture of the resulting $\mathrm{Co}_{1-x} \mathrm{Ni}_{x} \mathrm{Si}_{2}{ }^{44}$ As it was discussed in Section III A, both $\mathrm{NiSi}_{2}$ and $\mathrm{CoSi}_{2}$ can be grown epitaxially on $\mathrm{Si}(001)$ using a variety of techniques because of their $\mathrm{CaF}_{2}$ structure which is very similar to that of Si. However, when these silicides are formed through a standard solid-state reaction between a thin $\mathrm{Ni}$ or Co film and $\mathrm{Si}(001)$, only $\mathrm{NiSi}_{2}$ forms epitaxially while $\mathrm{CoSi}_{2}$ (which has a slightly larger lattice mismatch of $1.23 \%$ with $\mathrm{Si}$ compared to $0.46 \%$ for $\mathrm{NiSi}_{2}$ ) forms a polycrystalline film with different epitaxial components. ${ }^{67}$ By studying the texture of $\mathrm{Co}_{1-x} \mathrm{Ni}_{x} \mathrm{Si}_{2}$ films formed through a solid state reaction between a $\mathrm{Co}(\mathrm{Ni})$ alloy and $\mathrm{Si}(001)$, Smeets et al. investigated the quality of the resulting epitaxial film as a function of its lattice constant by introducing increasing amounts of $\mathrm{Ni}$ in the starting $\mathrm{Co}(\mathrm{Ni})$ alloy. As continuous addition of $\mathrm{Ni}$ is expected to generate a monotonic change in the lattice constant of the final $\mathrm{Co}_{1-x} \mathrm{Ni}_{x} \mathrm{Si}_{2}$ film from that of $\mathrm{CoSi}_{2}$ to that of $\mathrm{NiSi}_{2}$, Smeets et al. 
expected to see a gradual increase in epitaxial quality by adding more $\mathrm{Ni}$ to the deposited $\mathrm{Co}(\mathrm{Ni})$ alloy.

In order to quantify the epitaxial quality of the film with increasing $\mathrm{Ni}$ content, the RBS channeling yield was measured as a function of the $\mathrm{Ni}$ content in the $\mathrm{Co}(\mathrm{Ni})$ alloy along with some high-resolution pole figures measured on selected samples (see Fig. 27). Based on these measurements, Smeets et al. were able to divide the texture evolution of the $\mathrm{Co}_{1-x} \mathrm{Ni}_{x} \mathrm{Si}_{2}$ films with increasing $\mathrm{Ni}$ content into three regions. First, for Ni concentrations ranging between $0 \%$ and $15 \%$, the addition of Ni gradually changes the typical polycrystalline axiotaxy $\mathrm{CoSi}_{2}$ texture ${ }^{67}$ to an epitaxially textured $\mathrm{Co}_{1-x} \mathrm{Ni}_{x} \mathrm{Si}_{2}$ film where the $\{110\}$ planes are aligned with the substrate (evidenced by the epitaxial spots visible in the $15 \%$ pole figure of Fig. 27 which can be explained with this orientation). This epitaxial alignment however differs from the expected $\mathrm{NiSi}_{2}$-type epitaxy where a $\{001\}$ plane is parallel to the interface. Second, increasing the Ni concentration from $15 \%$ to $40 \%$ destroys the $\{110\}$ epitaxial orientation and a randomly oriented film is obtained for a $\mathrm{Ni}$ concentration of $40 \%$ (evidenced by the featureless $40 \%$ pole figure in Fig. 27). Third, increasing the $\mathrm{Ni}$ concentration beyond $50 \%$ and thus forming a Ni-rich $\mathrm{Co}_{1-x} \mathrm{Ni}_{x} \mathrm{Si}_{2}$ film results in a gradually improving epitaxial $\{001\}$ orientation as is expected for a $\mathrm{NiSi}_{2}$ film.

Based solely on geometrical considerations and the improving lattice match between $\mathrm{Co}_{1-x} \mathrm{Ni}_{x} \mathrm{Si}_{2}$ and $\mathrm{Si}$ with increasing $\mathrm{Ni}$ content, a steady improvement of the $\mathrm{Co}_{1-x} \mathrm{Ni}_{x} \mathrm{Si}_{2}\{001\}$ oriented epitaxial quality of the film should be observed. ${ }^{44}$ Thus, to explain the dominance of the $\{110\}$ epitaxial orientation for low $\mathrm{Ni}$ concentrations

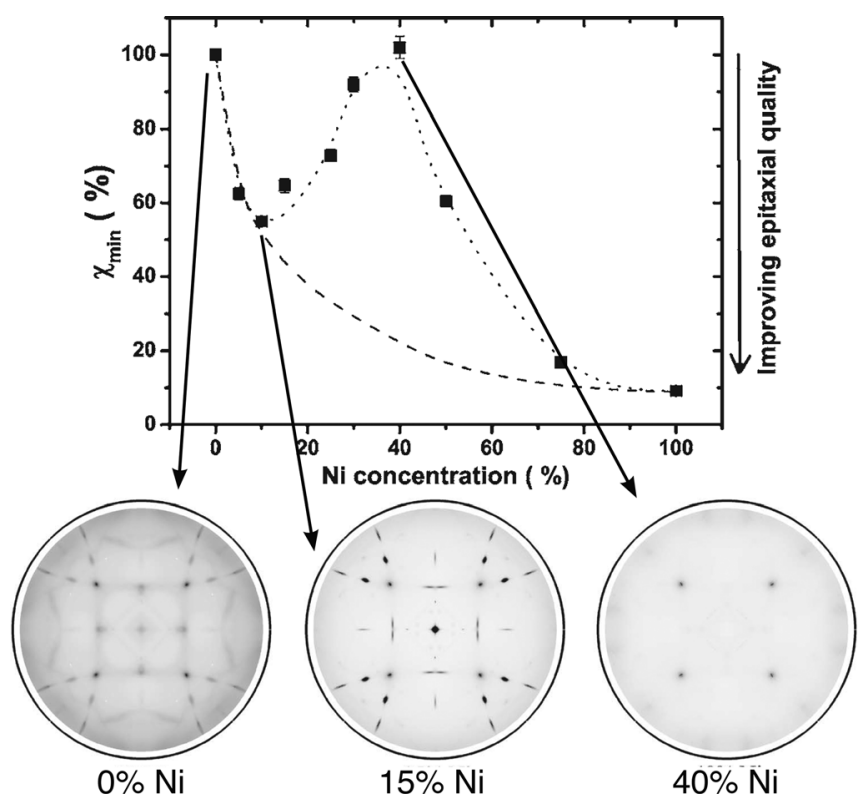

FIG. 27. RBS channeling yield for $\mathrm{Co}_{1-x} \mathrm{Ni}_{x} \mathrm{Si}_{2}$ films on $\mathrm{Si}(001)$ as a function of the $\mathrm{Ni}$ concentration in the deposited $\mathrm{Co}(\mathrm{Ni})$ alloy along with highresolution pole figures measured on samples with $\mathrm{Ni}$ concentrations of $0 \%$, $15 \%$, and $40 \%$. The dotted line in the RBS channeling yield plot is a guide to the eye while the dashed line depicts the expected improvement in epitaxial quality with increasing $\mathrm{Ni}$ concentration when only changes in lattice matching are taken into account. Figures reprinted with permission from J. Appl. Phys. 103, 063506 (2008). Copyright 2008 AIP Publishing LLC. (between $0 \%$ and 15\%) and the deterioration of the epitaxial quality between $15 \%$ and $40 \%$, Smeets et al. looked into the growth kinetics during $\mathrm{Co}_{1-x} \mathrm{Ni}_{x} \mathrm{Si}_{2}$ formation. These kinetics were already investigated in detail by D'Heurle et al. ${ }^{163}$ in the mid 1980s and showed that the preferred nucleation sites of $\mathrm{Co}_{1-x} \mathrm{Ni}_{x} \mathrm{Si}_{2}$ were located at different locations in the film for different $\mathrm{Ni}$ concentrations in the deposited $\mathrm{Co}(\mathrm{Ni})$ alloy. A summary of these positions is provided in Fig. 28. For a low $\mathrm{Ni}$ concentration $(\leq 15 \%)$, the film consists of $\mathrm{CoSi}$ prior to the formation of the $\mathrm{Co}_{1-x} \mathrm{Ni}_{x} \mathrm{Si}_{2}$ disilicide phase. Due to the immiscibility of $\mathrm{CoSi}$ and $\mathrm{NiSi}$, the $\mathrm{Ni}$ is in solution and is found near the interface with the Si substrate. As the disilicide phase nucleates at that interface, ${ }^{44}$ a preferential orientation with respect to the substrate is enabled for the growing grains (see Fig. 28(a)). Smeets et al. proposed that the dominance of $\{110\}$ oriented grains arises from a lower nucleation temperature and a larger lateral growth rate compared to grains belonging to the other texture components. For Ni concentrations between $15 \%$ and $40 \%$, $\mathrm{NiSi}$ and $\mathrm{CoSi}$ are both present prior to $\mathrm{Co}_{1-x} \mathrm{Ni}_{x} \mathrm{Si}_{2}$ formation due to their immiscibility. D'Heurle et al. observed that $\mathrm{NiSi}$ and $\mathrm{CoSi}$ are present in a bilayer structure with $\mathrm{NiSi}$ near the interface with the Si substrate and $\mathrm{CoSi}$ at the surface of the film. Surprisingly, they found that the formation of $\mathrm{Co}_{1-x} \mathrm{Ni}_{x} \mathrm{Si}_{2}$ primarily happens in the interface region between the two monosilicide phases away from the Si substrate (see Fig. 28(b)). The nucleation at this interface can be attributed to the fact that the nucleation barrier is the smallest where the inhomogeneity in the metal distribution is the largest. ${ }^{44,163,164}$ As this interface region is not in direct contact with the Si substrate, the nucleating grains can only develop a preferential orientation related to that of the $\mathrm{CoSi}$ and $\mathrm{NiSi}$ films, not directly to that of the $\mathrm{Si}(001)$ substrate. Increasing the $\mathrm{Ni}$ content in this concentration range gradually widens and moves the $\mathrm{CoSi} / \mathrm{NiSi}$ interface region away from the substrate, resulting in an increasingly random texture as more and more $\mathrm{Co}_{1-x} \mathrm{Ni}_{x} \mathrm{Si}_{2}$ grains will nucleate in that region. For Ni-rich concentrations (i.e., $>50 \% \mathrm{Ni}$ ), the NiSi/ $\mathrm{CoSi}$ interface region keeps moving further towards the surface and becomes smaller. This allows an increasing amount of Ni-rich $\mathrm{Co}_{1-x} \mathrm{Ni}_{x} \mathrm{Si}_{2}$ grains to form in the NiSi region at the interface (see Fig. 28(c)) with the typical $\mathrm{NiSi}_{2}\{001\}$ epitaxial orientation, which amounts to the improving

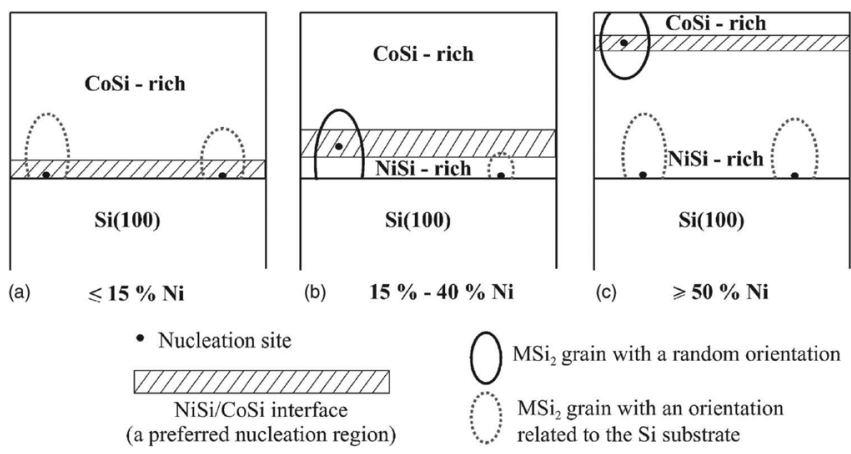

FIG. 28. Schematic of the position of the preferred $\mathrm{Co}_{1-x} \mathrm{Ni}_{x} \mathrm{Si}_{2}$ nucleation sites for three different $\mathrm{Ni}$ concentration ranges: (a) $\leq 15 \%$; (b) $15 \%-40 \%$; (c) $>50 \%$. Figure reprinted with permission from J. Appl. Phys. 103, 063506 (2008). Copyright 2008 AIP Publishing LLC. 
epitaxial quality of the $\mathrm{Co}_{1-x} \mathrm{Ni}_{x} \mathrm{Si}_{2}$ film when approaching the pure $100 \% \mathrm{Ni}$ case.

From the discussion of the two examples above, it is clear that the use of soluble alloying elements during silicide formation greatly influences the texture of the resulting silicide phase. When trying to explain the observed influence on texture, it is clear that both the change in unit cell dimensions of the silicide due to the incorporation of the alloying element as well as the kinetics and redistribution of the alloying element during the solid-state reaction have to be taken into account. Intuitively, a similar influence of alloying on the texture of germanides can be expected, although literature reports on this subject have not been found at the time of writing.

\section{Altered kinetics by adding insoluble elements: Alloys and interlayers}

Elements added to the metal-silicon/germanium system that are not significantly soluble in the targeted silicide/germanide are not expected to have an influence on the unit cell dimensions of that phase. As such, the texture of the targeted phase will mainly be influenced by altered formation kinetics due to the presence of the insoluble third element, as it can, e.g., influence grain boundary or interface energies and grain boundary diffusion by residing at the grain boundaries of the silicide/germanide or at the interface between the silicide/ germanide and the substrate.

In 2004, Özcan et al. published a detailed study on the texture of $\mathrm{CoSi}_{2}$ that was formed through the solid-state reaction between a $\mathrm{Si}(001)$ substrate and a thin Co film alloyed with $5 \% \mathrm{Ti}^{66}{ }^{66}$ a concentration that is well above the solubility limit of $\mathrm{Ti}$ in $\mathrm{CoSi}$ and $\mathrm{CoSi}_{2}$. The upper part of Fig. 29 shows the in situ XRD measurements performed on both a pure Co film and a $\mathrm{Co}(5 \% \mathrm{Ti})$ alloy, which reveal a significant influence of the presence of $\mathrm{Ti}$ on the phase formation. From these measurements, it can be observed that the formation temperature of the three cobalt silicides, i.e., $\mathrm{Co}_{2} \mathrm{Si}, \mathrm{CoSi}$ and $\mathrm{CoSi}_{2}$, is shifted to higher temperatures when formed from the $\mathrm{Co}(5 \% \mathrm{Ti})$ alloy. Furthermore, the intensity of the $\mathrm{CoSi}_{2}(220)$ peak is significantly higher in the case of the $\mathrm{Co}(5 \% \mathrm{Ti})$ alloy (the maximum intensity in the lower in situ XRD pattern is about 3.5 times higher than in the upper one), indicating that the two films exhibit a different microstructure and overall texture.

The texture of the $\mathrm{CoSi}_{2}$ formed from a pure Co film and a $\mathrm{Co}(5 \% \mathrm{Ti})$ alloy was measured using high-resolution pole figures (lower part of Fig. 29). A complete overview of the different texture components present in a pure $\mathrm{CoSi}_{2}$ on $\mathrm{Si}(001)$ has been published by De Keyser et al. ${ }^{67}$ and was summarized in Section III D 2. From their observations, Özcan et al. observed that the addition of 5\% Ti resulted in an increased intensity of the axiotaxy component that is related to the alignment of the $\mathrm{CoSi}_{2}\{110\}$ planes that are perpendicular to the substrate surface (i.e., those having poles with $\chi=90^{\circ}$ ) with $\mathrm{Si}\{110\}$ planes. Furthermore, the increased number of $\mathrm{CoSi}_{2}$ grains having their (110) plane parallel to the substrate, as suggested by the in situ XRD results, was confirmed by the pole figure measurements

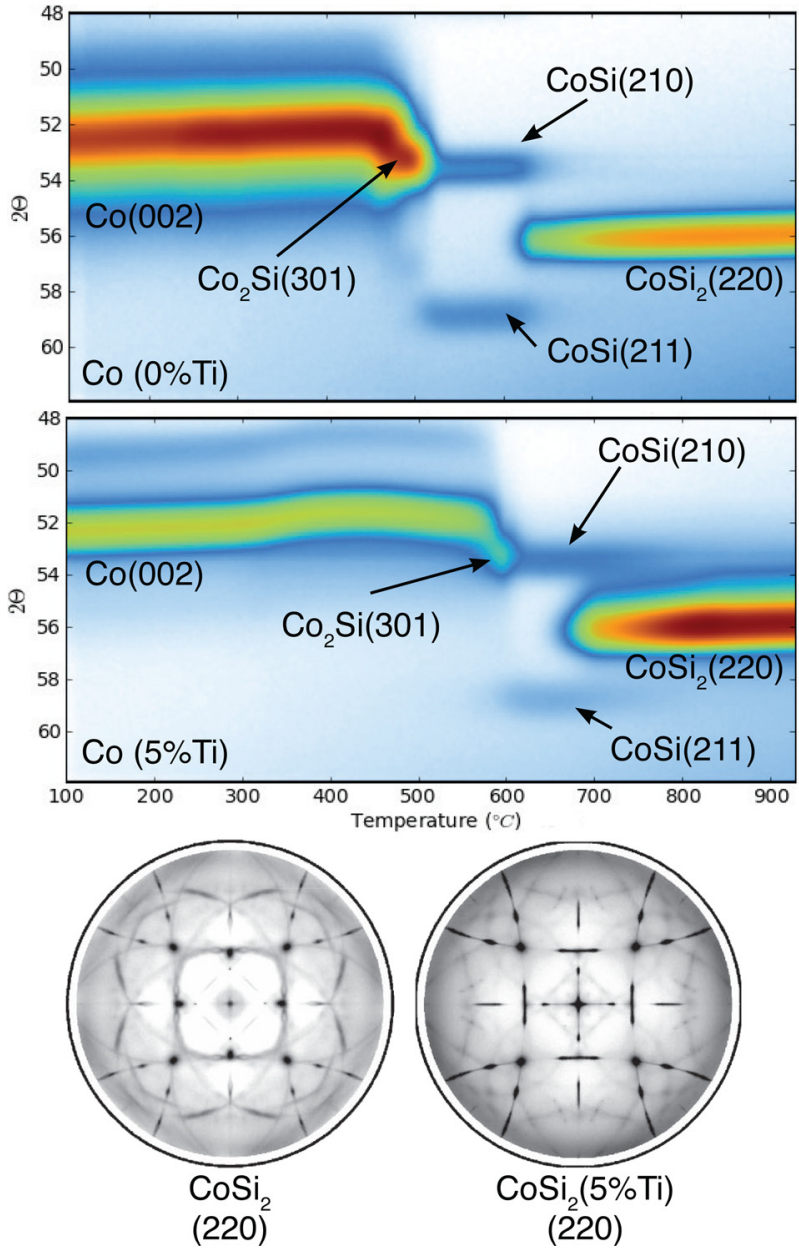

FIG. 29. Top: In situ XRD measurements for the formation of $\mathrm{CoSi}_{2}$ from a pure Co film and a Co film alloyed with $5 \% \mathrm{Ti}$. The initial Co and Co(Ti) layers had a thickness of $20 \mathrm{~nm}$ and were annealed at a rate of $3{ }^{\circ} \mathrm{C} / \mathrm{s}$ to $950{ }^{\circ} \mathrm{C}$ to form $\mathrm{CoSi}_{2}{ }^{66}$ Bottom: $\mathrm{CoSi}_{2}(220)$ pole figures recorded on a $\mathrm{CoSi}_{2}$ film formed from pure Co (left) and $\mathrm{Co}(5 \% \mathrm{Ti})$ (right). Pole figures reprinted with permission from J. Appl. Phys. 95, 8376 (2004). Copyright 2004 AIP Publishing LLC.

which showed an increased intensity for the spots belonging to an epitaxial component with $\mathrm{CoSi}_{2}(110) / / \mathrm{Si}(001) .{ }^{66}$ Özcan et al. proposed different growth kinetics for the different texture components as an explanation for the observed change in relative volume fractions of the texture components between pure $\mathrm{CoSi}_{2}$ and $\mathrm{CoSi}_{2}$ formed from a $\mathrm{Co}(5 \% \mathrm{Ti})$ alloy. Because the presence of Ti pushes the nucleation temperature of $\mathrm{CoSi}_{2}$ to a higher temperature (due to the influence on grain boundary and/or interface energy), the growth of the competing texture components at this elevated temperature can be expected to result in a change of relative volume fractions if their growth kinetics differ. The fact that growth kinetics can be different between texture components of a single silicide/germanide phase has also been observed in the case of $\mathrm{C} 54-\mathrm{TiSi}_{2} .{ }^{30}$

In the early 1990s, an even more pronounced influence of $\mathrm{Ti}$ on the texture of $\mathrm{CoSi}_{2}$ was observed when a thin layer of $\mathrm{Ti}$ was introduced as an interlayer between the Co film and the $\mathrm{Si}$ substrate. Annealing such a $\mathrm{Co} / \mathrm{Ti} / \mathrm{Si}$ stack resulted in the formation of a thin, single crystal, epitaxial $\mathrm{CoSi}_{2}$ layer. As epitaxial silicides were an important study 
subject at that time, this process was investigated further and was named titanium mediated epitaxy ${ }^{95,165}$ (TIME) (see also Section III A). In the mid 1990s, Tung et al. discovered that an interlayer of $\mathrm{SiO}_{2}$ (the native oxide forming on $\mathrm{Si}$ ) has a similar effect and even leads to better quality epitaxial $\mathrm{CoSi}_{2}$ films than in the case of a Ti interlayer. This technique was termed oxide mediated epitaxy (OME). ${ }^{94,95}$ The epitaxial disilicide formation achieved using TIME and OME can be linked to the altered phase formation sequence. Indeed, epitaxial $\mathrm{CoSi}_{2}$ formation was also observed either using lowrate reactive deposition of $\mathrm{Co}$ onto a $\mathrm{Si}$ substrate, thereby skipping the metal-rich phases in the formation sequence, or by depositing Co atoms sufficiently slowly onto a heated $\mathrm{Si}(001)$ substrate (on which epitaxial $\mathrm{CoSi}_{2}$ formation is impossible using a simple solid-phase reaction), which also resulted in the direct formation of epitaxial $\mathrm{CoSi}_{2} \cdot{ }^{91,166}$ The standard OME technique allowed for the growth of only a very thin layer of epitaxial $\mathrm{CoSi}_{2}(\sim 1-3 \mathrm{~nm})$, which was then used as a template layer for MBE growth of a thicker film of epitaxial $\mathrm{CoSi}_{2}$. It was observed later that the use of a $\mathrm{Ti}$ capping layer on top of the $\mathrm{Co} / \mathrm{SiO}_{x} / \mathrm{Si}$ stack allowed for the formation of thicker layers of epitaxial $\mathrm{CoSi}_{2}$ $(\sim 10-30 \mathrm{~nm}) .^{95,167-169}$

Subsequently, Detavernier et al. observed that the use of interlayers of Mo or $\mathrm{Cr},{ }^{96}$ as well as alloys or interlayers of Ta or $\mathrm{W}^{97}$ induces similar effects on the texture of the resulting $\mathrm{CoSi}_{2}$ which grows with a strong preferential (110) and (100) orientation (i.e., the $\mathrm{CoSi}_{2}$ grains preferentially grow with their (110) or (100) plane parallel to the substrate). Based on their results, they proposed a mechanism explaining the influence of these interlayers on the $\mathrm{CoSi}_{2}$ texture. For very thin interlayers, the insoluble element will be present on the grain boundaries of the preceding $\mathrm{CoSi}$ phase, influencing the grain boundary energy and thus the $\mathrm{CoSi}_{2}$ nucleation which can result in a growth with a preferential orientation. Thicker interlayers will act as a diffusion barrier, limiting the flux of Co atoms diffusing towards the substrate. In this way, the formation of $\mathrm{CoSi}$ is inhibited and $\mathrm{CoSi}_{2}$ can form as the first phase at the interface in contact with the single crystal Si substrate. ${ }^{97}$ The presence of these two regimes was observed for all the elements studied. Not surprisingly, as diffusion properties depend on materials, the interlayer thickness for which the system moves from one regime to the other was found to depend on the element studied.

In the Ni-Si system, Deduytsche et al. reported in 2007 that the use of $\mathrm{W}$, which is insoluble in $\mathrm{NiSi}$, as an alloying element influences the texture of $\mathrm{NiSi}$ formed from a thin $\mathrm{Ni}(\mathrm{W})$ alloy. They observed that using small amounts of $\mathrm{W}$ $(\sim 2 \%)$ already significantly decreases the intensity of the axiotaxial NiSi texture components, while alloying with $7 \%$ $\mathrm{W}$ or more changes the overall texture of the resulting $\mathrm{NiSi}$ film from axiotaxy to epitaxy. However, an explanation as to why the observed epitaxial texture component forms was lacking and is still an open question today. ${ }^{39}$

\section{E. Texture inheritance from a precursor phase}

In this final section, we discuss texture inheritance, a phenomenon in which the texture of a targeted silicide/ germanide is influenced by a strong, distinct texture of the phase from which it forms. An example of this can be found in the case of silicide formation from a thin amorphous mixture of $\mathrm{Ni}$ with 40 at. \% of Si. In 2008, De Keyser et al. observed that annealing such a layer on a $\mathrm{Si}(001)$ or (111) substrate leads to the formation of an epitaxially aligned hexagonal $\theta$ phase as the first forming phase instead of the combination of different Ni-rich silicides that form for a pure $\mathrm{Ni}$ film ${ }^{46}$ (see also Section IV A for details on the phase formation for a pure $\mathrm{Ni}$ film on $\mathrm{Si}$ ). This can be seen in the in situ XRD measurements for both a pure $\mathrm{Ni}$ film and a $\mathrm{Ni}(40 \%$ $\mathrm{Si})$ mixture on $\mathrm{Si}(001)$ displayed in Fig. 30 . The fact that hardly any diffraction peaks are visible in the measurement for the $\mathrm{Ni}(40 \% \mathrm{Si})$ mixture before the formation of NiSi suggests the presence of a highly epitaxial phase. Pole figure measurements along with EBSD allowed De Keyser et al. to identify this phase as the $\theta$ phase ${ }^{46}$ growing with a strong

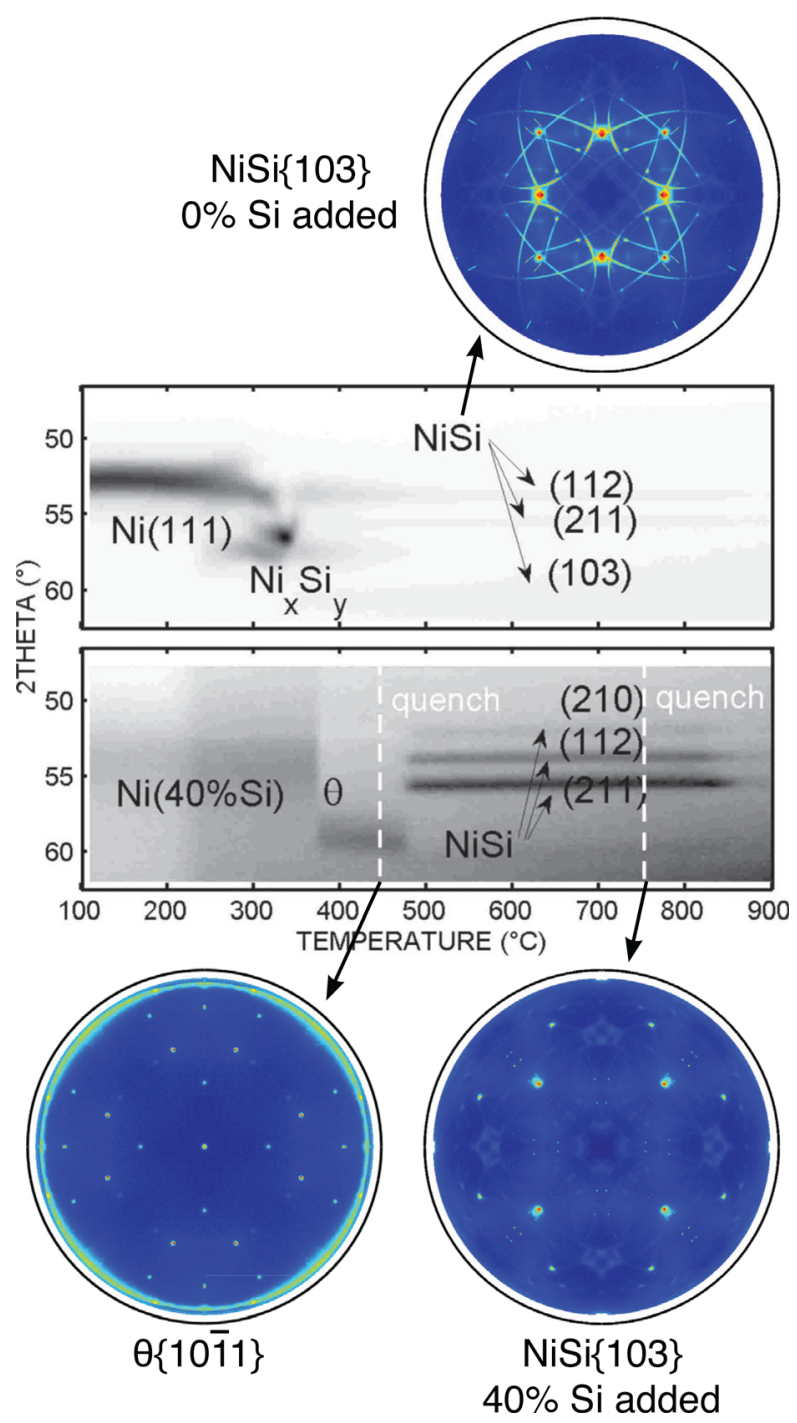

FIG. 30. In situ XRD measurement of the formation of NiSi from a $10 \mathrm{~nm}$ $\mathrm{Ni}$ (top) or $10 \mathrm{~nm} \mathrm{Ni}\left(40 \% \mathrm{Si}\right.$ ) (bottom) film, through a ramp anneal at $3{ }^{\circ} \mathrm{C} / \mathrm{s}$ on a (001) oriented Silicon-On-Insulator (SOI) substrate. Pole figures for the $\mathrm{NiSi}$ phase in both cases and for the $\theta$ phase forming from the $\mathrm{Ni}(40 \% \mathrm{Si})$ film are included. In situ XRD measurements reprinted with permission from Appl. Phys. Lett. 94, 033504 (2009). Copyright 2009 AIP Publishing LLC. 
epitaxial texture as evidenced by the clear diffraction spots visible in the $\theta\{101\}$ pole figure in Fig. 30 .

In 2009, Van Bockstael et al. ${ }^{43}$ found that the texture of the NiSi phase that forms from the epitaxial $\theta$ phase in the case of a $\mathrm{Ni}(40 \% \mathrm{Si})$ film on $\mathrm{Si}(001)$ or (111) is very different to the texture of $\mathrm{NiSi}$ when formed from a pure Ni layer. On both substrates, the final NiSi displays an epitaxial alignment with large grains instead of the typical axiotaxy texture that is expected for NiSi growing from a pure Ni film (see Section IV A), which is illustrated through the $\mathrm{NiSi}\{103\}$ pole figures shown in Fig. 30 for NiSi formed on a $\mathrm{Si}(001)$ substrate. Van Bockstael et al. argued that the peculiar epitaxial texture of $\mathrm{NiSi}$ formed from a $\mathrm{Ni}(40 \% \mathrm{Si})$ mixture must be a consequence of the presence of the epitaxial $\theta$ phase from which it forms. Otherwise, one would expect to observe this epitaxial $\mathrm{NiSi}$ component also in NiSi forming from a pure Ni layer, which is not the case (see Section III D 3). Furthermore, it was pointed out that similarities between the crystal structures of the $\theta$ phase and NiSi suggest that the formation of $\mathrm{NiSi}$ out of $\theta$ only requires a relatively minor rearrangement of the $\mathrm{Si}$ atoms, implying that the $\mathrm{Si}$ sublattice can remain more or less stationary during the transformation of $\theta$ into NiSi. This could provide a mechanism through which the epitaxial alignment of the $\theta$ phase can be inherited by $\mathrm{NiSi}$, explaining the observed epitaxial NiSi texture. On Si(111), Van Bockstael et al. observed that the epitaxial orientation of the $\theta$ phase and NiSi are indeed closely related, supporting the proposed mechanism of texture inheritance. However, on $\mathrm{Si}(001)$ no such simple relation between the two epitaxial alignments could be identified, showing that in this case the inheritance mechanism is more complex (and is still an open question). In addition, the inherited epitaxial texture of the NiSi phase was found to have an important impact on the morphological stability of the film. Van Bockstael et al. observed that the agglomeration temperature for an epitaxial $\mathrm{NiSi}$ film formed from a $\mathrm{Ni}(40 \% \mathrm{Si})$ mixture is significantly higher than for a NiSi film with the typical axiotaxy texture formed from a pure Ni layer (about $100^{\circ} \mathrm{C}$ higher for a $3{ }^{\circ} \mathrm{C} / \mathrm{s}$ anneal). This observation enhances our argument put forward in Section IV $\mathrm{C}$ that the presence of axiotaxy promotes agglomeration.

More recently, a similar mechanism of texture inheritance has been put forward by Gaudet et al. to explain the observed fiber texture of the $\theta$ phase during the reaction of a pure Ni film with $\mathrm{Si}(001)$. According to Gaudet et al., the $\theta$ phase inherits this fiber texture from the preceding $\delta-\mathrm{Ni}_{2} \mathrm{Si}$ phase, which also exhibits a fiber texture. Furthermore, it was observed in this study that the well-known axiotaxy texture of the NiSi phase is convoluted with a broad fiber texture. Gaudet et al. argued that the NiSi inherits this broad fiber from either the $\theta$ or the $\delta-\mathrm{Ni}_{2} \mathrm{Si}$ phase, as this latter phase was observed to re-appear right before $\mathrm{NiSi}$ growth starts.

\section{SUMMARY AND CONCLUSIONS}

Thin films of silicides and germanides formed through a solid-state reaction between a thin metal film and a single crystal silicon or germanium substrate are widely studied in the microelectronics research community as they have an important application as electrical contacts in both present and future microelectronic devices. An important consequence of forming such thin films on a single crystal substrate is that the film grains can develop a preferential orientation with respect to the substrate during growth. This preferential grain orientation is referred to as the texture of the film. In this review, we provided an overview of the research that has been performed over the past decades targeting this texture in thin silicide/germanide films.

As the study of texture in such films initially relies on the ability to perform a measurement, we first discussed the two most important techniques that are used in modern texture studies, i.e., synchrotron based X-ray pole figures and electron backscattered diffraction. The first technique provides a straightforward way for a qualitative identification of the different phases and texture components that are present in the film, while the latter technique can provide a more quantitative picture of the different texture components and of the microstructure (grain size, grain morphology, etc.) of the film.

In Section III, we provided a historical overview of how texture in these films was addressed during the early years of silicide research, when focus was mainly directed towards the growth of epitaxial silicides. At this point, we discussed the driving forces for texture development in such films. It was argued that a minimization of interface energy drives the grains in the film to grow with a preferential orientation that results in a periodic interface structure in either one (axiotaxy) or two (epitaxy) dimension(s). It was pointed out that in the case of axiotaxy, plane alignment across the interface results in a periodic interface structure in one dimension, the periodic nature of which is preserved irrespective of interfacial curvature. For an epitaxial alignment, the periodic interface structure in one or both of the two dimensions can also be the result of plane alignment, in which case the epitaxy is more stable than when the periodicity within the interface plane is not caused by plane alignment.

The remainder of the review was aimed at providing a structured overview of texture research that has been published since the early 2000s, when the technique of highresolution synchrotron based X-ray pole figure measurements was introduced. It was pointed out that texture measurements are often crucial to unambiguously identify the correct phase formation sequence during silicide/germanide formation, as highly textured phases are easily overlooked using standard measurement techniques. The influence of texture on different important thin film properties such as phase stability, morphological stability, and electrical properties were discussed. Importantly, it was argued that the agglomeration of a thin silicide/germanide film is promoted if axiotaxy is the dominant texture type.

As many properties of thin silicide/germanide films are influenced by their microstructure, it is important to understand the factors that can affect film texture. One can then exploit these and attempt to control the texture of these films. As an example, alloying of the binary films with a third element that is either soluble or insoluble in the targeted silicide/germanide has proven to effectively alter the final 
texture. Although a few detailed studies probing the influence of alloying elements on silicide texture have been performed and discussed in this review, open questions on how different alloying elements impact texture development (and hence the phase formation sequence) during silicide formation still remain. Furthermore, the impact of alloying on the texture of germanide films is a research area still to be explored.

${ }^{1}$ K. Maex and M. Van Rossum, Properties of Metal Silicides (Inspec, 1995).

${ }^{2}$ M. A. Nicolet and S. S. Lau, "Formation and characterization of transition-metal silicides," in VLSI Electronics: Microstructure Science (Academic Press, 1986).

${ }^{3}$ C. M. Osburn, "Silicides," in Rapid Thermal Processing Science and Technology, edited by R. B. Fair (Academic Press, 1993).

${ }^{4}$ L. Miglio and F. d'Heurle, Silicides: Fundamentals and Applications (World Scientific, 1999).

${ }^{5}$ C. Lavoie, F. M. d'Heurle, and S.-L. Zhang, "Silicides," in Handbook of Semiconductor Manufacturing Technology, edited by Y. Nishi and R. Doering (CRC Press, 2007).

${ }^{6}$ L.-J. Chen, Silicide Technology for Integrated Circuits, edited by L.-J. Chen (The Institution of Electrical Engineers, 2004).

${ }^{7}$ S.-L. Zhang and M. Östling, "Metal silicides in CMOS technology: Past, present, and future trends," Crit. Rev. Solid State Mater. Sci. 28, 1-129 (2003)

${ }^{8}$ J. P. Gambino and E. G. Colgan, "Silicides and ohmic contacts," Mater. Chem. Phys. 52, 99-146 (1998).

${ }^{9}$ S. P. Murarka, "Silicide thin films and their applications in microelectronics," Intermetallics 3, 173-186 (1995).

${ }^{10}$ S. P. Murarka, "Refractory silicides for integrated circuits," J. Vac. Sci. Technol. 17, 775 (1980).

${ }^{11}$ G. V. Naik, V. M. Shalaev, and A. Boltasseva, "Alternative plasmonic materials: Beyond gold and silver," Adv. Mater. 25, 3264-3294 (2013).

${ }^{12}$ A. L. Schmitt, J. M. Higgins, J. R. Szczech, and S. Jin, "Synthesis and applications of metal silicide nanowires," J. Mater. Chem. 20, 223-235 (2009).

${ }^{13} \mathrm{H}$. Kleinke, "New bulk materials for thermoelectric power generation: Clathrates and complex antimonides," Chem. Mater. 22, 604-611 (2009).

${ }^{14}$ R. Pretorius, C. C. Theron, A. Vantomme, and J. W. Mayer, "Compound phase formation in thin film structures," Crit. Rev. Solid State Mater. Sci. 24, 1-62 (1999).

${ }^{15}$ S. P. Murarka, Silicides for VLSI Applications (Academic Press, 1983).

${ }^{16}$ F. M. d'Heurle, "Silicide interfaces in silicon technology," J. Electron. Mater. 27, 1138-1147 (1998).

${ }^{17}$ K. Maex, "Silicides for integrated circuits: $\mathrm{TiSi}_{2}$ and $\mathrm{CoSi}_{2}$," Mater. Sci. Eng., R 11, vii-153 (1993).

${ }^{18}$ C. Lavoie, F. M. d'Heurle, C. Detavernier, and C. Cabral, "Towards implementation of a nickel silicide process for CMOS technologies," Microelectron. Eng. 70, 144-157 (2003).

${ }^{19}$ D. Mangelinck, J. Y. Dai, J. S. Pan, and S. K. Lahiri, "Enhancement of thermal stability of NiSi films on (100)Si and (111)Si by Pt addition," Appl. Phys. Lett. 75, 1736-1738 (1999).

${ }^{20}$ H. Yu, M. Schaekers, E. Rosseel, A. Peter, J. G. Lee, W. B. Song, D. S, T. Chiarella, L. A. Ragnarsson, S. Kubicek, J. L. Everaert, N. Horiguchi, K. Barla, D. Kim, A. V. Y. Thean, N. Collaert, and K. De Meyer, "1.5 $\times 10^{9} \Omega \mathrm{cm}^{2}$ contact resistivity on highly doped Si:P using Ge pre-amorphization and Ti silicidation," in 2015 International ELectron Devices Meeting (2015).

${ }^{21}$ C. H. Lin, B. Greene, S. Narasimha, J. Cai, A. Bryant, C. Radens, V. Narayanan, B. Linder, H. Ho, A. Aiyar, E. Alptekin, J.-J. An, M. Aquilino, R. Bao, V. Basker, N. Breil, M. Brodsky, W. Chang, L. Clevenger, D. Chidambarrao, C. Christiansen, D. Conklin, C. DeWan, H. Dong, L. Economikos, B. Engel, S. Fang, D. Ferrer, A. Friedman, A. Gabor, F. Guarin, X. Guan, M. Hasanuzzaman, J. Hong, D. Hoyos, B. Jagannathan, S. Jain, S.-J. Jeng, J. Johnson, B. Kannan, Y. Ke, B. Khan, B. Kim, S. Koswatta, A. Kumar, T. Kwon, U. Kwon, L. Lanzerotti, H.-K. Lee, W.-H. Lee, A. Levesque, W. Li, Z. Li, W. Liu, S. Mahajan, K. McStay, H. Nayfeh, W. Nicoll, G. Northrop, A. Ogino, C. Pei, S. Polvino, R. Ramachandran, Z. Ren, R. Robison, I. Saraf, V. Sardesai, S. Saudari, D. Schepis, C. Sheraw, S. Siddiqui, L. Song, K. Stein, C. Tran, H. Utomo, R. Vega, G. Wang, H. Wang, W. Wang, X. Wang, D. Wehelle-Gamage,
E. Woodard, Y. Xu, Y. Yang, N. Zhan, K. Zhao, C. Zhu, K. Boyd, E. Engbrecht, K. Henson, E. Kaste, S. Krishnan, E. Maciejewski, H. Shang, N. Zamdmer, R. Divakaruni, J. Rice, S. Stiffler, and P. Agnello, "High performance $14 \mathrm{~nm}$ SOI FinFET CMOS technology with $0.0174 \mu \mathrm{m}^{2}$ embedded DRAM and 15 levels of Cu metallization," in 2014 IEEE International Electron Devices Meeting (IEDM) (IEEE, 2014), pp. 3.8.1-3.8.3.

${ }^{22} \mathrm{R}$. Pillarisetty, "Academic and industry research progress in germanium nanodevices," Nature 479, 324-328 (2011).

${ }^{23}$ S. Gaudet, C. Detavernier, A. J. Kellock, P. Desjardins, and C. Lavoie, "Thin film reaction of transition metals with germanium," J. Vac. Sci. Technol. A 24, 474 (2006).

${ }^{24}$ H.-J. Bunge, Texture Analysis in Materials Science, Mathematical Methods (Butterworths, London, 1982).

${ }^{25}$ U. F. Kocks, C. N. Tomé, and H. R. Wenk, Texture and Anisotropy, Preferred Orientations in Polycrystals and their Effect on Materials Properties (Cambridge University Press, 2000).

${ }^{26} \mathrm{~V}$. Randle and O. Engler, Introduction to Texture Analysis: Macrotexture, Microtexture, and Orientation Mapping, 2nd ed. (CRC Press, 2009).

${ }^{27}$ L. C. Feldman and J. W. Mayer, Fundamentals of Surface and Thin Film Analysis, 1st ed. (Prentice Hall, 1986).

${ }^{28}$ L. G. Schulz, "A direct method of determining preferred orientation of a flat reflection sample using a Geiger counter x-ray spectrometer," J. Appl. Phys. 20, 1030-1033 (1949).

${ }^{29}$ C. W. T. Bulle-Lieuwma, A. H. van Ommen, J. Hornstra, and C. N. A. M. Aussems, "Observation and analysis of epitaxial growth of $\mathrm{CoSi}_{2}$ on (100) Si,” J. Appl. Phys. 71, 2211 (1992).

${ }^{30}$ A. S. Özcan, K. F. Ludwig Jr, P. Rebbi, C. Lavoie, C. Cabral, and J. M. E. Harper, "Texture of $\mathrm{TiSi}_{2}$ thin films on Si (001)," J. Appl. Phys. 92, 5011 (2002).

${ }^{31}$ C. Detavernier, A. S. Özcan, J. Jordan-Sweet, E. A. Stach, J. Tersoff, F. M. Ross, and C. Lavoie, "An off-normal fibre-like texture in thin films on single-crystal substrates," Nature 426, 641-645 (2003).

${ }^{32} \mathrm{~J}$. J. Heizmann and C. Laruelle, "Simultaneous measurement of several X-ray pole figures,” J. Appl. Crystallogr. 19, 467-472 (1986).

${ }^{33}$ S. Gaudet, P. Desjardins, and C. Lavoie, "The thermally-induced reaction of thin Ni films with Si: Effect of the substrate orientation," J. Appl. Phys. 110, 113524 (2011).

${ }^{34}$ K. De Keyser, B. De Schutter, C. Detavernier, V. Machkaoutsan, M. Bauer, S. G. Thomas, J. Jordan-Sweet, and C. Lavoie, "Phase formation and texture of nickel silicides on $\mathrm{Si}_{1-x} \mathrm{C}_{x}$ epilayers," Microelectron. Eng. 88, 536-540 (2011).

${ }^{35}$ S. Gaudet, C. Coia, P. Desjardins, and C. Lavoie, "Metastable phase formation during the reaction of $\mathrm{Ni}$ films with $\mathrm{Si}(001)$ : The role of texture inheritance," J. Appl. Phys. 107, 093515 (2010).

${ }^{36}$ K. De Keyser, C. Van Bockstael, R. L. Van Meirhaeghe, C. Detavernier, E. Verleysen, H. Bender, W. Vandervorst, J. Jordan-Sweet, and C. Lavoie, "Phase formation and thermal stability of ultrathin nickelsilicides on Si(100),” Appl. Phys. Lett. 96, 173503 (2010).

${ }^{37}$ S. Gaudet, K. De Keyser, S. Lambert-Milot, J. Jordan-Sweet, C. Detavernier, C. Lavoie, and P. Desjardins, "Three dimensional reciprocal space measurement by $\mathrm{x}$-ray diffraction using linear and area detectors: Applications to texture and defects determination in oriented thin films and nanoprecipitates," J. Vac. Sci. Technol. A 31, 021505 (2013).

${ }^{38}$ C. Mocuta, M.-I. Richard, J. Fouet, S. Stanescu, A. Barbier, C. Guichet, O. Thomas, S. Hustache, A. V. Zozulya, and D. Thiaudière, "Fast pole figure acquisition using area detectors at the DiffAbs beamlineSynchrotron SOLEIL," J. Appl. Crystallogr. 46, 1842-1853 (2013).

${ }^{39} \mathrm{~K}$. De Keyser, "Texture of thin silicide and germanide films," Ph.D. thesis (Ghent University, 2011).

${ }^{40}$ Electron Backscatter Diffraction in Materials Science, edited by A. J. Schwartz, M. Kumar, B. L. Adams, and D. P. Field (Springer US, Boston, MA, 2009).

${ }^{41}$ K. De Keyser, C. Detavernier, and R. L. Van Meirhaeghe, "Characterization of the texture of silicide films using electron backscattered diffraction," Appl. Phys. Lett. 90, 121920 (2007).

${ }^{42}$ K. De Keyser, R. L. Van Meirhaeghe, C. Detavernier, J. Jordan-Sweet, and C. Lavoie, "Texture of cobalt germanides on $\mathrm{Ge}(100)$ and $\mathrm{Ge}(111)$ and its influence on the formation temperature," J. Electrochem. Soc. 157, H395-H404 (2010).

${ }^{43}$ C. Van Bockstael, K. De Keyser, R. L. Van Meirhaeghe, C. Detavernier, J. Jordan-Sweet, and C. Lavoie, "Influence of a transient hexagonal phase on the microstructure and morphological stability of NiSi films," Appl. Phys. Lett. 94, 033504 (2009). 
${ }^{44}$ D. Smeets, A. Vantomme, K. De Keyser, C. Detavernier, and C. Lavoie, "The role of lattice mismatch and kinetics in texture development: $\mathrm{Co}_{1-x} \mathrm{Ni}_{x} \mathrm{Si}_{2}$ thin films on Si(100)," J. Appl. Phys. 103, 063506 (2008).

${ }^{45}$ C. Van Bockstael, K. De Keyser, D. Deduytsche, R. L. Van Meirhaeghe, C. Detavernier, J. Jordan-Sweet, and C. Lavoie, "Effect of Pt addition on growth stress and thermal stress of NiSi films," J. Appl. Phys. 104, 053510 (2008).

${ }^{46}$ K. De Keyser, C. Van Bockstael, C. Detavernier, R. L. Van Meirhaeghe, J. Jordan-Sweet, and C. Lavoie, "Epitaxial formation of a metastable hexagonal nickel-silicide,” Electrochem. Solid State 11, H266 (2008).

${ }^{47}$ H. Föll, P. S. Ho, and K. N. Tu, "Transmission electron microscopy of the formation of nickel silicides," Philos. Mag. A 45, 31-47 (1981).

${ }^{48}$ V. Teodorescu, L. Nistor, H. Bender, A. Steegen, A. Lauwers, K. Maex, and J. Van Landuyt, "In situ transmission electron microscopy study of Ni silicide phases formed on (001) Si active lines," J. Appl. Phys. 90, 167-174 (2001).

${ }^{49}$ A. Alberti, C. Bongiorno, B. Cafra, G. Mannino, E. Rimini, T. Metzger, C. Mocuta, T. Kammler, and T. Feudel, "Pseudoepitaxial transrotational structures in $14 \mathrm{~nm}$-thick NiSi layers on [001] silicon," Acta Crystallogr. B: Struct. Sci. 61, 486-491 (2005).

${ }^{50} \mathrm{C}$. Detavernier and C. Lavoie, "Influence of Pt addition on the texture of NiSi on Si(001),” Appl. Phys. Lett. 84, 3549-3551 (2004).

${ }^{51}$ C. Detavernier, J. Jordan-Sweet, and C. Lavoie, "Texture of NiSi films on Si(001), (111), and (110) substrates,” J. Appl. Phys. 103, 113526 (2008).

${ }^{52}$ K.-N. Tu, E. I. Alessandrini, W.-K. Chu, H. Krautle, and J. W. Mayer, "Epitaxial growth of nickel silicide $\mathrm{NiSi}_{2}$ on silicon," Jpn. J. Appl. Phys., Part 1 13, 669 (1974).

${ }^{53}$ H. Ishiwara, K. Hikosaka, M. Nagatomo, and S. Furukawa, "Characterization of epitaxial metal silicide films grown on silicon," Surf. Sci. 86, 711-717 (1979).

${ }^{54}$ S. Saitoh, H. Ishiwara, T. Asano, and S. Furukawa, "Single crystalline silicide formation,” Jpn. J. Appl. Phys., Part 1 20, 1649-1656 (1981).

${ }^{55}$ M. S. Fung and H. C. Cheng, "Localized epitaxial growth of C54 and C49 $\mathrm{TiSi}_{2}$ on (111)Si,” Appl. Phys. Lett. 47, 1312-1314 (1985).

${ }^{56}$ I. C. Wu and J. J. Chu, "Local epitaxy of $\mathrm{TiSi}_{2}$ on (111)Si: Effects due to rapid thermal annealing and to the annealing atmosphere," J. Appl. Phys. 60, 3172-3175 (1986).

${ }^{57}$ J. J. Chu and I. C. Wu, "Transmission electron microscope study of the growth kinetics of $\mathrm{TiSi}_{2}$ epitaxy on (111)Si," J. Appl. Phys. 61, 549-551 (1987).

${ }^{58}$ A. Catana, P. E. Schmid, M. Heintze, F. Lévy, P. Stadelmann, and R. Bonnet, "Atomic scale study of local $\mathrm{TiSi}_{2} / \mathrm{Si}$ epitaxies," J. Appl. Phys. 67, 1820-1825 (1990).

${ }^{59}$ W.-K. Wan and S.-T. Wu, "Texture of titanium self-aligned silicide (salicide)," Scr. Mater. 35, 53-58 (1996).

${ }^{60}$ W.-K. Wan and S.-T. Wu, "Epitaxial $\mathrm{TiSi}_{2}$ on silicon by rapid thermal annealing," Mater. Lett. 30, 105-108 (1997).

${ }^{61}$ L.-M. Wang and S.-T. Wu, "Epitaxial growth of $\mathrm{TiSi}_{2}$ (C49) on (001)Si by rapid thermal annealing," Jpn. J. Appl. Phys. 36, 6475-6480 (1997).

${ }^{62}$ V. Svilan, K. P. Rodbell, L. A. Clevenger, C. Cabral, R. A. Roy, C. Lavoie, J. Jordan-Sweet, and J. M. E. Harper, "Crystallographic texture of C54 titanium disilicide as a function of deep submicron structure geometry," J. Electron. Mater. 26, 1090-1095 (1997).

${ }^{63}$ F. La Via, V. Raineri, M. G. Grimaldi, L. Miglio, M. Iannuzzi, F. Marabelli, S. Bocelli, S. Santucci, and A. R. Phani, "Role of the substrate in the C49-C54 transformation of $\mathrm{TiSi}_{2}$," J. Vac. Sci. Technol. B 18, 721-728 (2000).

${ }^{64}$ S. M. Yalisove, R. T. Tung, and D. Loretto, "Epitaxial orientation and morphology of thin $\mathrm{CoSi}_{2}$ films grown on $\mathrm{Si}(100)$ : Effects of growth parameters," J. Vac. Sci. Technol. A 7, 1472-1474 (1989).

${ }^{65}$ J. Zegenhagen, J. R. Patel, P. E. Freeland, and R. T. Tung, "Co on Si(111) - Silicide Formation,” Phys. Rev. B 44, 13626-13630 (1991).

${ }^{66}$ A. S. Özcan, K. F. Ludwig Jr, C. Detavernier, C. Lavoie, and J. JordanSweet, "Axiotaxy of $\mathrm{CoSi}_{2}$ thin films on $\mathrm{Si}(100)$ substrates and the effects of Ti alloying," J. Appl. Phys. 95, 8376 (2004).

${ }^{67}$ K. De Keyser, C. Detavernier, J. Jordan-Sweet, and C. Lavoie, "Texture of $\mathrm{CoSi}_{2}$ films on $\mathrm{Si}(111)$, (110) and (001) substrates," Thin Solid Films 519, 1277-1284 (2010).

${ }^{68}$ N. Jedrecy, Y. Zheng, A. Waldhauer, M. Sauvage-Simkin, and R. Pinchaux, "Epitaxy of $\beta$-FeSi ${ }_{2}$ on Si(111)," Phys. Rev. B 48, 8801-8808 (1993).

${ }^{69}$ C. Detavernier, C. Lavoie, J. Jordan-Sweet, and A. S. Özcan, "Texture of tetragonal $\alpha-\mathrm{FeSi}_{2}$ films on $\mathrm{Si}(001)$,” Phys. Rev. B 69, 174106 (2004).
${ }^{70}$ R. W. Bower, D. Sigurd, and R. E. Scott, "Formation kinetics and structure of $\mathrm{Pd}_{2} \mathrm{Si}$ films on Si," Solid-State Electronics 16, 1461-1471 (1973).

${ }^{71}$ W. D. Buckley and S. C. Moss, "Structure and electrical characteristics of epitaxial palladium silicide contacts on single crystal silicon and diffused P-N diodes," Solid-State Electron. 15, 1331-1337 (1972).

${ }^{72}$ G. A. Hutchins and A. Shepela, "Growth and Transformation of $\mathrm{Pd}_{2} \mathrm{Si}$ on (111), (110), and (100) Si," Thin Solid Films 18, 343-363 (1973).

${ }^{73}$ T. Kawamura, D. Shinoda, and H. Muta, "Oriented growth of the interfacial PtSi layer or between Pt And Si," Appl. Phys. Lett. 11, 101-103 (1967).

${ }^{74}$ A. K. Sinha, R. B. Marcus, T. T. Sheng, and S. E. Haszko, "Thermal stability of thin PtSi films on silicon substrates," J. Appl. Phys. 43, 3637-3643 (1972).

${ }^{75}$ H. Ben Ghozlene, P. Beaufrère, and A. Authier, "Crystallography of PtSi films on (001) silicon,” J. Appl. Phys. 49, 3998-4004 (1978).

${ }^{76}$ C. Kumpf, R. Nicula, and E. Burkel, "Growth and structure of thin $\mathrm{Pt}_{2} \mathrm{Si}$ and PtSi layers on $\mathrm{Si}(111)$ and (001) characterized with in situ grazing incidence diffraction,” J. Appl. Crystallogr. 30, 1016-1021 (1997).

${ }^{77}$ M. Falke, H. Schletter, O. Filonenko, A. V. Mogilatenko, G. Beddies, S. Schulze, M. Hietschold, A. Bleloch, K. De Keyser, and C. Detavernier, "Axiotaxy of $\mathrm{CrSi}_{2}$ on $\mathrm{Si}(001)$; from the micrometer- to the Angstromscale," Microsc. Microanal. 13, 396-397 (2007).

${ }^{78}$ A. Vantomme, M. F. Wu, S. Hogg, U. Wahl, W. Deweerd, H. Pattyn, G. Langouche, S. Jin, and H. Bender, "Stabilisation and phase transformation of hexagonal rare-earth silicides on Si(111)," Nucl. Instrum. Methods Phys. Res. B 147, 261-266 (1999).

${ }^{79}$ J. A. Knapp and S. T. Picraux, "Epitaxial growth of rare-earth silicides on (111)Si,” Appl. Phys. Lett. 48, 466-468 (1986).

${ }^{80}$ S. L. Liew, B. Balakrisnan, C. S. Ho, O. Thomas, and D. Z. Chi, "Phase and texture of Er-Germanide formed on $\mathrm{Ge}(001)$ through a solid-state reaction," J. Electrochem. Soc. 154, H9-H12 (2007).

${ }^{81}$ F. Geenen, W. Knaepen, J. Demeulemeester, K. De Keyser, J. JordanSweet, C. Lavoie, A. Vantomme, and C. Detavernier, "On the formation and structural properties of hexagonal rare earth (Y, Gd, Dy, Er, and Yb) disilicide thin films," J. Alloys Compd. 611, 149-156 (2014).

${ }^{82}$ B. Balakrisnan, C. C. Tan, S. L. Liew, P. C. Lim, G. K. L. Goh, Y. L. Foo, and D. Z. Chi, "Texture of NiGe on $\mathrm{Ge}(001)$ and its evolution with formation temperature," Appl. Phys. Lett. 87, 241922 (2005).

${ }^{83}$ S. Gaudet, C. Detavernier, C. Lavoie, and P. Desjardins, "Reaction of thin Ni films with Ge: Phase formation and texture," J. Appl. Phys. 100, 034306 (2006).

${ }^{84}$ Y. F. Hsieh, E. D. Marshall, and S. S. Lau, "Partial epitaxial growth of cobalt germanides on (111)Ge,” Appl. Phys. Lett. 51, 1588 (1987).

${ }^{85}$ H. P. Sun, Y. B. Chen, X. Q. Pan, D. Z. Chi, R. Nath, and Y. L. Foo, "Formation and evolution of epitaxial $\mathrm{Co}_{5} \mathrm{Ge}_{7}$ film on $\mathrm{Ge}(001)$ surface by solid-state reaction in an in situ ultrahigh-vacuum transmission electron microscope," Appl. Phys. Lett. 87, 211909 (2005).

${ }^{86}$ Y. F. Hsieh, "Interfacial reactions of palladium thin films on $\mathrm{Ge}(111)$ and Ge(001)," Thin Solid Films 162, 295-303 (1988).

${ }^{87}$ F. Geenen, W. Knaepen, K. De Keyser, K. Opsomer, R. Vanmeirhaeghe, J. Jordan-Sweet, C. Lavoie, and C. Detavernier, "Formation and texture of palladium germanides studied by in situ X-ray diffraction and pole figure measurements," Thin Solid Films 551, 86-91 (2014).

${ }^{88}$ Y. F. Hsieh, "Interfacial reactions of platinum thin films on (111) and (001) germanium,” J. Appl. Phys. 63, 1177 (1988).

${ }^{89}$ R. T. Tung, J. M. Poate, J. C. Bean, J. M. Gibson, and D. C. Jacobson, "Epitaxial silicides," Thin Solid Films 93, 77-90 (1982).

${ }^{90}$ K. N. Tu, "Epitaxial growth of transition-metal silicides on silicon," Mater. Sci. Rep. 6, 53-140 (1991).

${ }^{91}$ A. Vantomme, S. Degroote, J. Dekoster, G. Langouche, and R. Pretorius, "Concentration-controlled phase selection of silicide formation during reactive deposition,” Appl. Phys. Lett. 74, 3137-3139 (1999).

${ }^{92}$ R. T. Tung, J. M. Gibson, and J. M. Poate, "Growth of single-crystal epitaxial silicides on silicon by the use of template layers," Appl. Phys. Lett. 42, 888-890 (1983).

${ }^{93}$ M. L. A. Dass, D. B. Fraser, and C. S. Wei, "Growth of epitaxial CoSi ${ }_{2}$ on (100)Si," Appl. Phys. Lett. 58, 1308-1310 (1991).

${ }^{94}$ R. T. Tung, "Oxide mediated epitaxy of $\mathrm{CoSi}_{2}$ on silicon," Appl. Phys. Lett. 68, 3461 (1996).

${ }^{95} \mathrm{R}$. T. Tung and S. Ohmi, "Epitaxial silicide interfaces in microelectronics," Thin Solid Films 369, 233-239 (2000).

${ }^{96}$ C. Detavernier, R. L. Van Meirhaeghe, F. Cardon, K. Maex, H. Bender, B. Brijs, and W. Vandervorst, "Formation of epitaxial $\mathrm{CoSi}_{2}$ by a $\mathrm{Cr}$ or 
Mo interlayer: Comparison with a Ti interlayer," J. Appl. Phys. 89, 2146-2150 (2001)

${ }^{97}$ C. Detavernier, C. Lavoie, and R. L. Van Meirhaeghe, "CoSi 2 formation in the presence of Ti, Ta or W," Thin Solid Films 468, 174-182 (2004).

${ }^{98}$ A. E. White, K. T. Short, R. C. Dynes, J. P. Garno, and J. M. Gibson, "Mesotaxy: Formation of buried single-crystal $\mathrm{CoSi}_{2}$ layers by implantation," MRS Proc. 74, 481 (1986).

${ }^{99}$ S. Mantl, "Ion beam synthesis of epitaxial silicides: Fabrication, characterization and applications," Mater. Sci. Rep. 8, 1-95 (1992).

${ }^{100}$ S. Mantl and H. L. Bay, "New method for epitaxial heterostructure layer growth," Appl. Phys. Lett. 61, 267-269 (1992)

${ }^{101}$ S. Mantl, "Molecular beam allotaxy: A new approach to epitaxial heterostructures," J. Phys. D: Appl. Phys. 31, 1-17 (1998).

${ }^{102}$ Y. F. Hsieh, E. D. Marshall, and S. S. Lau, "Partial epitaxial growth of $\mathrm{Ni}_{2} \mathrm{Ge}$ and NiGe on Ge(111)," Thin Solid Films 162, 287-294 (1988).

${ }^{103}$ B. De Schutter, K. Van Stiphout, N. M. Santos, E. Bladt, J. Jordan-Sweet, S. Bals, C. Lavoie, C. M. Comrie, A. Vantomme, and C. Detavernier, "Phase formation and texture of thin nickel germanides on $\mathrm{Ge}(001)$ and Ge(111),”J. Appl. Phys. 119, 135305 (2016).

${ }^{104}$ M. D. Tucker, P. O. A. Persson, M. C. Guenette, J. Rosén, M. M. M. Bilek, and D. R. McKenzie, "Substrate orientation effects on the nucleation and growth of the $\mathrm{M}_{n+1} \mathrm{AX}_{n}$ phase $\mathrm{Ti}_{2} \mathrm{AlC}$," J. Appl. Phys. 109, 014903 (2011)

${ }^{105}$ S. Lambert-Milot, S. Gaudet, C. Lacroix, D. Ménard, R. A. Masut, C. Lavoie, and P. Desjardins, "MnP nanoclusters embedded in $\mathrm{GaP}$ epitaxial films grown by organometallic vapor-phase epitaxy: A reciprocal space mapping and transmission electron microscopy study," J. Vac. Sci. Technol. A 30,061510 (2012).

${ }^{106}$ F. M. d'Heurle, "Nucleation of a new phase from the interaction of two adjacent phases,” J. Mater. Res. 3, 167-195 (1988).

${ }^{107}$ M. I. Richard, J. Fouet, M. Texier, C. Mocuta, C. Guichet, and O. Thomas, "Continuous and collective grain rotation in nanoscale thin films during silicidation,” Phys. Rev. Lett. 115, 266101-266105 (2015).

${ }^{108}$ Silicide Technology for Integrated Circuits, edited by L.-J. Chen (The Institution of Engineering and Technology, 2009).

${ }^{109}$ J. M. E. Harper, C. Cabral, Jr., and C. Lavoie, "Mechanisms for enhanced formation of the C54 phase of titanium silicide ultra-large-scale integration contacts," Annu. Rev. Mater. Sci. 30, 523-543 (2000).

${ }^{110}$ D. Deduytsche, C. Detavernier, R. L. Van Meirhaeghe, and C. Lavoie, "High-temperature degradation of NiSi films: Agglomeration versus $\mathrm{NiSi}_{2}$ nucleation,” J. Appl. Phys. 98, 033526 (2005).

${ }^{111}$ A. Alberti, C. Spinella, A. La Magna, and E. Rimini, "Nucleation and growth of NiSi from $\mathrm{Ni}_{2} \mathrm{Si}$ transrotational domains," Appl. Phys. Lett. 90, 053507-053504 (2007).

${ }^{112}$ A. Alberti and A. La Magna, "Role of the early stages of Ni-Si interaction on the structural properties of the reaction products," J. Appl. Phys. 114, 121301 (2013)

${ }^{113}$ C. Detavernier, C. Lavoie, and F. M. d'Heurle, "Thermal expansion of the isostructural PtSi and NiSi: Negative expansion coefficient in NiSi and stress effects in thin films," J. Appl. Phys. 93, 2510 (2003).

${ }^{114}$ F. M. d'Heurle, C. S. Petersson, J. E. E. Baglin, S. J. La Placa, and C. Y. Wong, "Formation of thin films of NiSi: Metastable structure, diffusion mechanisms in intermetallic compounds," J. Appl. Phys. 55, 4208-4218 (1984).

${ }^{115}$ H. von Känel, "Growth and characterization of epitaxial Ni and Co silicides," Mater. Sci. Rep. 8, 193-269 (1992).

${ }^{116}$ M. Tinani, A. Mueller, Y. Gao, E. A. Irene, Y. Z. Hu, and S. P. Tay, "In situ real-time studies of nickel silicide phase formation," J. Vac. Sci. Technol. B 19, 376-383 (2001).

${ }^{117}$ C. Lavoie, C. Coia, F. M. d'Heurle, C. Detavernier, C. Cabral, P. Desjardins, and A. J. Kellock, "Reactive diffusion in the Ni-Si system: Phase sequence and formation of metal-rich phases," Defect Diffus. Forum 237-240, 825-836 (2005).

${ }^{118}$ C. Lavoie, C. Detavernier, C. Cabral, Jr., F. M. d'Heurle, A. J. Kellock, J. Jordan-Sweet, and J. M. E. Harper, "Effects of additive elements on the phase formation and morphological stability of nickel monosilicide films," Microelectron. Eng. 83, 2042-2054 (2006).

${ }^{119}$ P. Nash and A. Nash, "The Ni-Si (Nickel-Silicon) system," Bull. Alloy Phase Diagrams 8, 6-14 (1987).

${ }^{120}$ J. M. Gibson and J. L. Batstone, "In-situ transmission electron microscopy of $\mathrm{NiSi}_{2}$ formation by molecular beam epitaxy," Surf. Sci. 208, 317-350 (1989).

${ }^{121}$ B. De Schutter, K. van Stiphout, N. M. Santos, E. Bladt, J. Jordan-Sweet, S. Bals, C. Lavoie, C. M. Comrie, A. Vantomme, and C. Detavernier,
"Phase formation and texture of thin nickel germanides on $\mathrm{Ge}(001)$ and Ge(111),” J. Appl. Phys. 119, 135305 (2016).

${ }^{122}$ E. G. Colgan, J. P. Gambino, and Q. Z. Hong, "Formation and stability of silicides on polycrystalline silicon,” Mater. Sci. Eng., R 16, 43-96 (1996).

${ }^{123}$ K. De Keyser, C. Detavernier, R. L. Van Meirhaeghe, J. Jordan-Sweet, and C. Lavoie, "The texture of thin NiSi films and its effect on agglomeration," in Applications of Texture Analysis, edited by A. D. Rollett (John Wiley \& Sons, Inc., Hoboken, NJ, USA, 2008), pp. 1-9.

${ }^{124}$ T. P. Nolan, R. Sinclair, and R. Beyers, "Modeling of agglomeration in polycrystalline thin films: Application to $\mathrm{TiSi}_{2}$ on a silicon substrate," J. Appl. Phys. 71, 720-6 (1992).

${ }^{125}$ R. T. Tung, "Recent advances in Schottky barrier concepts," Mater. Sci. Eng., R 35, 1-138 (2001).

${ }^{126}$ R. T. Tung, "The physics and chemistry of the Schottky barrier height," Appl. Phys. Rev. 1, 011304 (2014).

${ }^{127}$ N. F. Mott, "The theory of crystal rectifiers," Proc. R. Soc. London, Ser. A 171, 27-38 (1939).

${ }^{128}$ W. Schottky, "Zur Halbleitertheorie der Sperrschicht- und Spitzengleichrichter," Z. Phys. 113, 367-414 (1939).

${ }^{129}$ R. T. Tung, "Schottky-Barrier formation at single-crystal metal-semiconductor interfaces," Phys. Rev. Lett. 52, 461-464 (1984).

${ }^{130}$ R. J. Hauenstein, T. E. Schlesinger, T. C. McGill, B. D. Hunt, and L. J. Schowalter, "Schottky barrier height measurements of epitaxial $\mathrm{NiSi}_{2}$ on Si," Appl. Phys. Lett. 47, 853 (1985).

${ }^{131}$ M. Ospelt, J. Henz, L. Flepp, and H. von Känel, "Schottky barriers of epitaxial $\mathrm{NiSi}_{2}$ on $\mathrm{Si}(111)$," Appl. Phys. Lett. 52, 227 (1988).

${ }^{132}$ J. Vrijmoeth, J. F. van der Veen, D. R. Heslinga, and T. M. Klapwijk, "Medium-energy ion-scattering study of a possible relation between the Schottky-barrier height and the defect density at $\mathrm{NiSi}_{2} / \mathrm{Si}(111)$ interfaces," Phys. Rev. B 42, 9598-9608 (1990).

${ }^{133}$ R. T. Tung, A. Levi, J. P. Sullivan, and F. Schrey, "Schottky-Barrier inhomogeneity at epitaxial $\mathrm{NiSi}_{2}$ interfaces on $\mathrm{Si}(100)$," Phys. Rev. Lett. 66, 72-75 (1991).

${ }^{134}$ Y. P. Song, R. L. Van Meirhaeghe, W. H. Laflère, and F. Cardon, "On the difference in apparent barrier height as obtained from capacitancevoltage and current-voltage-temperature measurements on $\mathrm{Al} / \mathrm{p}$ - $\mathrm{InP}$ Schottky barriers," Solid-State Electron. 29, 633-638 (1986).

${ }^{135}$ R. T. Tung, "Electron transport at metal-semiconductor interfaces: General theory," Phys. Rev. B 45, 13509-13523 (1992).

${ }^{136}$ R. T. Tung, "Electron-transport of inhomogeneous Schottky Barriers," Appl. Phys. Lett. 58, 2821-2823 (1991).

${ }^{137}$ J. H. Werner and H. H. Güttler, "Temperature dependence of Schottky barrier heights on silicon,” J. Appl. Phys 73, 1315 (1993).

${ }^{138}$ L. D. Bell and W. J. Kaiser, "Observation of interface band structure by ballistic-electron-emission microscopy," Phys Rev Lett 61, 2368-2371 (1988).

${ }^{139}$ T. Meyer, H. Sirringhaus, and H. von Känel, "Studying interfaces on a nm scale by BEEM," Thin Solid Films 318, 195-200 (1998).

${ }^{140}$ E. Y. Lee, H. Sirringhaus, and H. von Känel, "Direct mapping of the $\mathrm{CoSi}_{2} / \mathrm{Si}(111)$ interface by ballistic-electron-emission microscopy and modulation spectroscopy," Phys. Rev. B 50, 14714-14717 (1994).

${ }^{141}$ E. Y. Lee, H. Sirringhaus, U. Kafader, and H. von Känel, "Ballisticelectron-emission-microscopy investigation of hot-carrier transport in epitaxial $\mathrm{CoSi}_{2}$ films on $\mathrm{Si}(100)$ and $\mathrm{Si}(111)$," Phys. Rev. B 52, 1816-1829 (1995).

${ }^{142} \mathrm{H}$. von Känel, T. Meyer, and M. Klemenc, "Ballistic-electron-emission microscopy on epitaxial silicides," Jpn. J. Appl. Phys., Part 1 37, 3800-3804 (1998).

${ }^{143}$ C. Detavernier, R. L. Van Meirhaeghe, R. Donaton, K. Maex, and F. Cardon, "Ballistic electron emission microscopy study of barrier height inhomogeneities introduced in Au/n-Si Schottky contacts by a HF pretreatment," J. Appl. Phys. 84, 3226 (1998).

${ }^{144}$ S. Zhu, X.-P. Qu, R. L. Van Meirhaeghe, C. Detavernier, G.-P. Ru, F. Cardon, and B.-Z. Li, "Ballistic electron emission microscopy studies of the temperature dependence of Schottky barrier height distribution in $\mathrm{CoSi}_{2} / \mathrm{n}-\mathrm{Si}(100)$ diodes formed by solid phase reaction," Solid-State Electron. 44, 2217-2223 (2000).

${ }^{145}$ C. Zaring, H. Jiang, B. G. Svensson, and M. Östling, "Boron redistribution during formation of nickel silicides," Appl. Surf. Sci. 53, 147-152 (1991).

${ }^{146}$ S. P. Murarka and D. S. Williams, "Dopant redistribution in silicidesilicon and silicide-polycrystalline silicon bilayered structures," J. Vac. Sci. Technol. B 5, 1674-1688 (1987).

${ }^{147}$ M. Wittmer, "Silicide contacts for shallow junction devices," Thin Solid Films 107, 99-110 (1983). 
${ }^{148}$ K. Hoummada, G. Tellouche, I. D. Blum, A. Portavoce, and D. Mangelinck, "Arsenic clustering during formation of the transient Ni silicide," Scr. Mater. 67, 169-172 (2012).

${ }^{149}$ F. Panciera, K. Hoummada, M. Gregoire, M. Juhel, N. Bicais, and D. Mangelinck, "Three dimensional distributions of arsenic and platinum within NiSi contact and gate of an n-type transistor," Appl. Phys. Lett. 99, 051911 (2011).

${ }^{150}$ O. Cojocaru-Mirédin, C. Perrin-Pellegrino, D. Mangelinck, and D. Blavette, "Boron redistribution during reactive diffusion in Ni-Si contacts," Microelectron. Eng. 87, 271-273 (2010).

${ }^{151} \mathrm{H}$. Kimura and R. Tomita, "Texture change of NiSi film with dopant implantation," in Interconnect Technology Conference and Materials for Advanced Metallization (IITC/MAM), 2011 (IEEE, 2011), pp. 1-3.

${ }^{152}$ M. Bauer, D. Weeks, Y. Zhang, and V. Machkaoutsan, "Tensile strained selective silicon carbon alloys for recessed source drain areas of devices," ECS Trans. 3, 187-196 (2006).

${ }^{153}$ V. Machkaoutsan, S. Mertens, M. Bauer, A. Lauwers, K. Verheyden, K. Vanormelingen, P. Verheyen, R. Loo, M. Caymax, S. Jakschik, D. Theodore, P. Absil, S. G. Thomas, and E. H. A. Granneman, "Improved thermal stability of Ni-silicides on Si:C epitaxial layers," Microelectron. Eng. 84, 2542-2546 (2007).

${ }^{154}$ J. Demeulemeester, D. Smeets, C. Van Bockstael, C. Detavernier, C. M. Comrie, N. P. Barradas, A. Vieira, and A. Vantomme, "Pt redistribution during Ni(Pt) silicide formation,” Appl. Phys. Lett. 93, 261912 (2008).

${ }^{155}$ J. Demeulemeester, D. Smeets, C. M. Comrie, C. Van Bockstael, W. Knaepen, C. Detavernier, K. Temst, and A. Vantomme, "The influence of Pt redistribution on $\mathrm{Ni}_{1-x} \mathrm{Pt}_{x} \mathrm{Si}$ growth properties," J. Appl. Phys. 108, 043505 (2010).

${ }^{156}$ J. Demeulemeester, D. Smeets, C. M. Comrie, N. P. Barradas, A. Vieira, C. Van Bockstael, C. Detavernier, K. Temst, and A. Vantomme, "On the growth kinetics of $\mathrm{Ni}(\mathrm{Pt})$ silicide thin films," J. Appl. Phys. 113, 163504 (2013).

${ }^{157}$ K. Hoummada, C. Perrin-Pellegrino, and D. Mangelinck, "Effect of Pt addition on Ni silicide formation at low temperature: Growth, redistribution, and solubility," J. Appl. Phys. 106, 063511 (2009).

${ }^{158}$ D. Mangelinck, K. Hoummada, A. Portavoce, C. Perrin, R. Daineche, M. Descoins, D. J. Larson, and P. H. Clifton, "Three-dimensional composition mapping of NiSi phase distribution and Pt diffusion via grain boundaries in $\mathrm{Ni}_{2} \mathrm{Si}$," Scr. Mater. 62, 568-571 (2010).

${ }^{159}$ M. Putero, L. Ehouarne, E. Ziegler, and D. Mangelinck, "First silicide formed by reaction of $\mathrm{Ni}(13 \% \mathrm{Pt})$ films with $\mathrm{Si}(100)$ : Nature and kinetics by in-situ X-ray reflectivity and diffraction," Scr. Mater. 63, 24-27 (2010).

${ }^{160}$ F. Panciera, D. Mangelinck, K. Hoummada, M. Texier, M. Bertoglio, A. De Luca, M. Gregoire, and M. Juhel, "Direct epitaxial growth of $\theta-\mathrm{Ni}_{2} \mathrm{Si}$ by reaction of a thin $\mathrm{Ni}(10$ at. \% Pt) film with $\mathrm{Si}(100)$ substrate," Scr. Mater. 78-79, 9-12 (2014).

${ }^{161}$ P. Adusumilli, L. J. Lauhon, D. N. Seidman, C. E. Murray, O. Avayu, and Y. Rosenwaks, "Tomographic study of atomic-scale redistribution of platinum during the silicidation of $\mathrm{Ni}_{0.95} \mathrm{Pt}_{0.05} / \mathrm{Si}(100)$ thin films," Appl. Phys. Lett. 94, 113103-113104 (2009).

${ }^{162}$ P. Adusumilli, D. N. Seidman, and C. E. Murray, "Silicide-phase evolution and platinum redistribution during silicidation of Ni0.95Pt0.05/ Si(100) specimens," J. Appl. Phys. 112, 064307-12 (2012).

${ }^{163}$ F. M. d'Heurle, D. D. Anfiteatro, V. R. Deline, and T. G. Finstad, "Reaction of silicon with films of Co-Ni alloys: Phase separation of the monosilicides and nucleation of the disilicides," Thin Solid Films 128, 107-124 (1985).

${ }^{164}$ C. Detavernier, X. P. Qu, R. L. Van Meirhaeghe, B. Z. Li, and K. Maex, "Mixing entropy and the nucleation of silicides: Ni-Pd-Si and Co-Mn-Si ternary systems," J. Mater. Res. 18, 1668-1678 (2003).

${ }^{165}$ R. T. Tung and F. Schrey, "Ti-interlayer mediated epitaxy of $\mathrm{CoSi}_{2}$ with Ti capping," MRS Proc. 402, 173 (1995).

${ }^{166}$ A. Vantomme, M. A. Nicolet, and N. D. Theodore, "Epitaxial CoSi 2 films on $\mathrm{Si}(100)$ by solid-phase reaction," J. Appl. Phys. 75, 3882-3891 (1994).

${ }^{167}$ G. B. Kim, J. S. Kwak, H. K. Baik, and S.-M. Lee, “Ex situ formation of oxide-interlayer-mediated-epitaxial $\mathrm{CoSi}_{2}$ film using Ti capping," J. Vac. Sci. Technol. B 17, 162-165 (1999).

${ }^{168}$ C. Detavernier, R. L. Van Meirhaeghe, F. Cardon, R. A. Donaton, and K. Maex, "CoSi 2 formation in the presence of interfacial silicon oxide," Appl. Phys. Lett. 74, 2930 (1999).

${ }^{169}$ C. Detavernier, R. L. Van Meirhaeghe, F. Cardon, and K. Maex, "CoSi 2 formation through $\mathrm{SiO}_{2}$," Thin Solid Films 386, 19-26 (2001). 